\title{
WestVirginiaUniversity
}

THE RESEARCH REPOSITORY @ WVU

Graduate Theses, Dissertations, and Problem Reports

2003

\section{Cellular specializations for sound localization}

\author{
Kevin C. Rowland \\ West Virginia University
}

Follow this and additional works at: https://researchrepository.wvu.edu/etd

\section{Recommended Citation}

Rowland, Kevin C., "Cellular specializations for sound localization" (2003). Graduate Theses, Dissertations, and Problem Reports. 1843.

https://researchrepository.wvu.edu/etd/1843

This Dissertation is protected by copyright and/or related rights. It has been brought to you by the The Research Repository @ WVU with permission from the rights-holder(s). You are free to use this Dissertation in any way that is permitted by the copyright and related rights legislation that applies to your use. For other uses you must obtain permission from the rights-holder(s) directly, unless additional rights are indicated by a Creative Commons license in the record and/ or on the work itself. This Dissertation has been accepted for inclusion in WVU Graduate Theses, Dissertations, and Problem Reports collection by an authorized administrator of The Research Repository @ WVU.

For more information, please contact researchrepository@mail.wvu.edu. 
Cellular Specializations for Sound Localization

Kevin C. Rowland

Dissertation submitted to the School of Medicine at West Virginia University in partial fulfillment of the requirements for the degree of

Doctor of Philosophy

\author{
George Spirou, Ph.D., Chair \\ Albert Berrebi, Ph.D. \\ Robert Goodman, Ph.D. \\ Ronald Millechia, Ph.D. \\ William Wonderlin, Ph.D.
}

Department of Physiology and Pharmacology

Morgantown, WV 2003

Keywords: sound localization, auditory brainstem, superior olivary complex, calyx of Held, mitochondria, potassium channels, inhibition 


\title{
ABSTRACT
}

\section{Cellular Specializations for Sound Localization}

\author{
Kevin C. Rowland
}

One of the key elements in auditory perception is the localization of sounds in space. The major cues used for localizing sounds in the azimuthal plane have long been recognized as interaural differences in time of arrival of a sound and amplitude differences between the two ears (Rayleigh 1907; Thompson 1878). High frequency sounds are reflected by the head and thereby produce interaural level differences (ILDs) that are used for localization. The head does not reflect low frequency sounds and so interaural timing differences (ITDs) are used. One of the cell groups of the auditory brainstem, the medial superior olive (MSO), functions in sound localization by comparing ITDs between the two ears. The MSO is defined as a binaural group of cells because it integrates input from the cochlear nucleus (CN) from each ear. Afferent nerve fibers from the ipsilateral $\mathrm{CN}$ are restricted to dendrites oriented laterally and inputs from the contralateral $\mathrm{CN}$ are segregated to medially oriented dendrites (Stotler 1953). At low to moderate sound levels, activation from each cochlear nucleus is below action potential threshold and MSO neurons only generate action potentials when inputs from both sides arrive within a short temporal window called the coincidence detection window.

Several cellular specializations exist along the auditory pathway that aid MSO cells in their ability to detect changes in ITD. These specializations include large nerve terminals and distinct organelle complexes located within terminals, which facilitate fast, well-timed inhibitory inputs to MSO cells. Very little is known about the role of inhibition in sound localization and proper understanding of its role depends on knowledge of the cells that impinge on the MSO and the pharmacology and kinetics of synaptic transmission in MSO cells. Also, the membranes of MSO cells contain specific voltage-gated potassium channels (Kv), these channels are known to affect membrane electrical properties, but how these channels influence ITD sensitivity is unknown. The main goal of my research was to understand these cellular specializations that contribute to neural processing of ITDs. 


\section{DEDICATION}

This dissertation is dedicated to my wife, Darcy. 


\section{ACKNOWLEDGEMENTS}

To my committee members Albert Berrebi, Robert Goodman, Ronald Millechia, and William Wonderlin, I would like to thank you for your time, scientific advice, patience and support.

I would especially like to thank my advisor, George Spirou for advice and encouragement throughout graduate school. 


\section{TABLE OF CONTENTS}

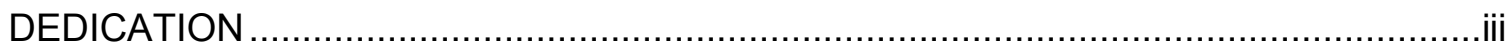

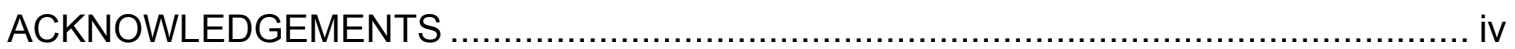

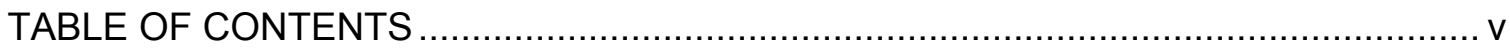

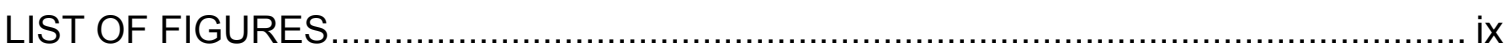

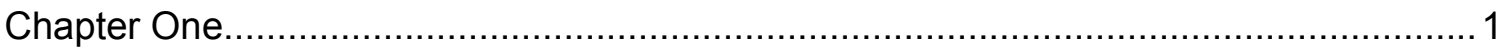

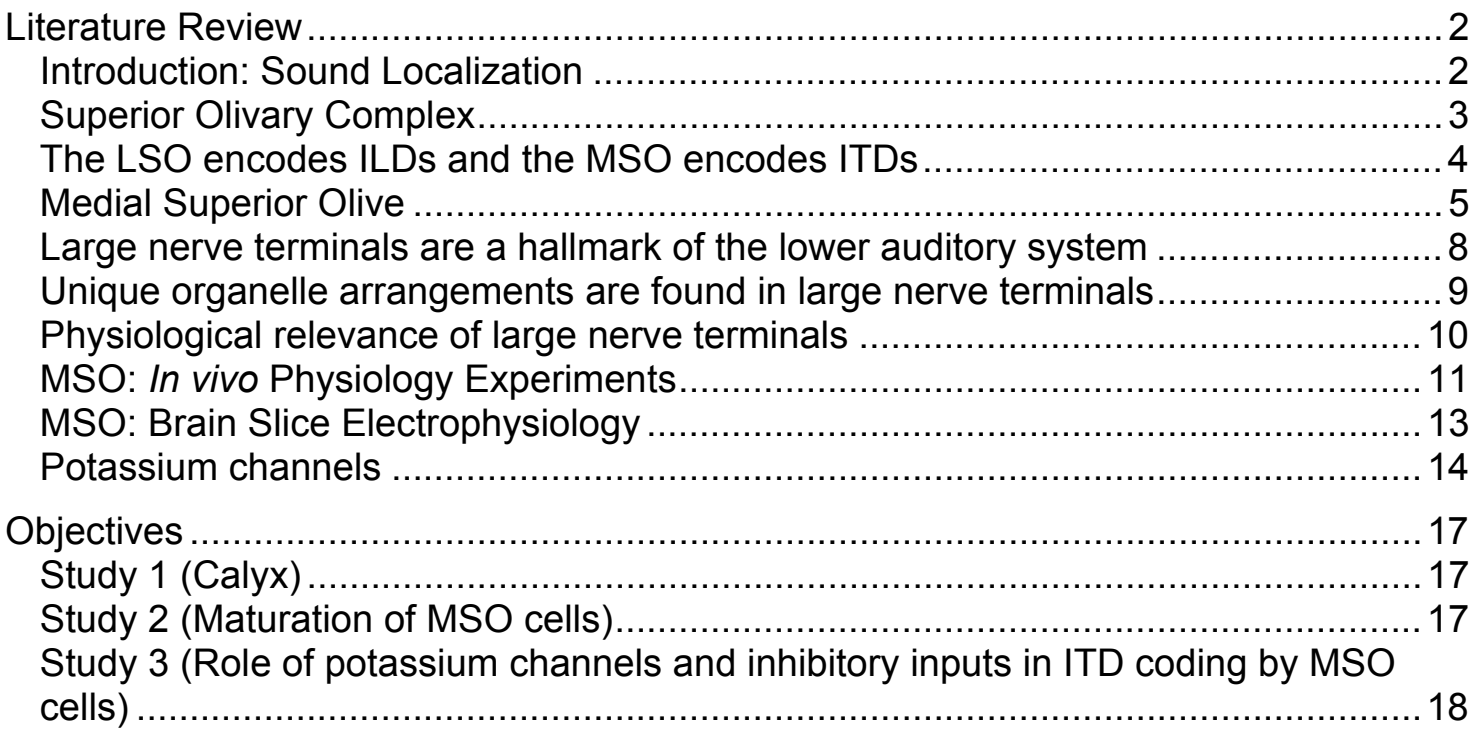

Chapter Two: Specialized Synapse-Associated Structures within the Calyx of Held .....20

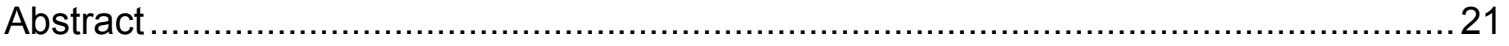

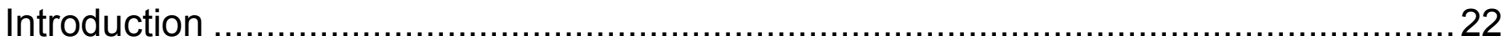

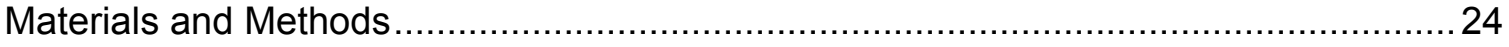

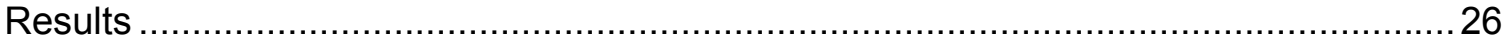

The calyx of Held is organized into distinct segments ............................................

Vesicular and tubular membrane structures in the nerve terminal............................ 30

Are there subpopulations of mitochondria in the calyx of Held? ............................. 31

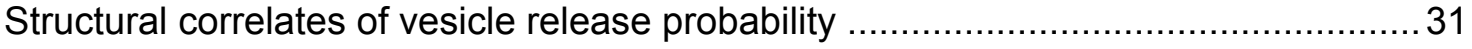




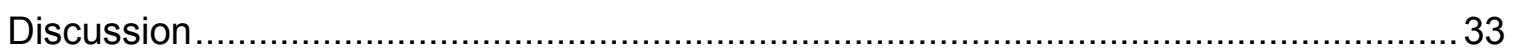

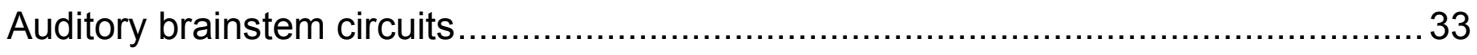

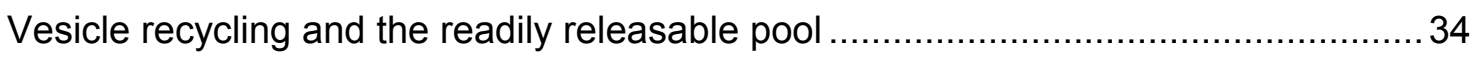

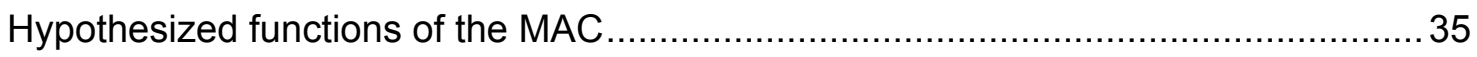

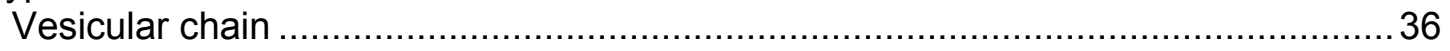

Mitochondrion, mitochondrial plaque, and filaments: a system to regulate

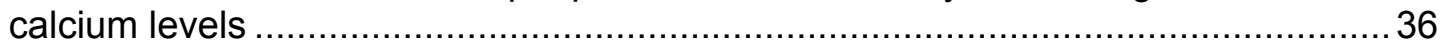

Mitochondrial geometry and cristae: an intracellular conduit for calcium movement 37

Mitochondrial biochemistry: possible source of energy and neurotransmitter ...........37

Punctum adherens: synaptic stabilization and development ............................... 38

Chapter 3: Maturation of membrane electrical properties of neurons in the medial

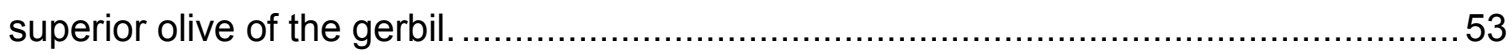

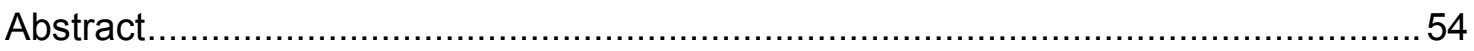

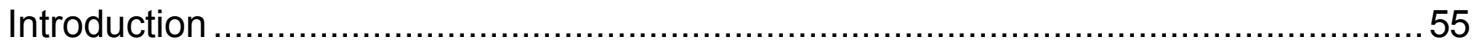

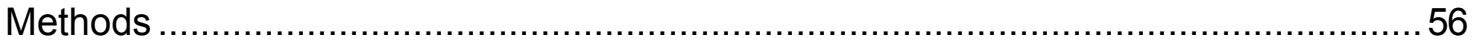

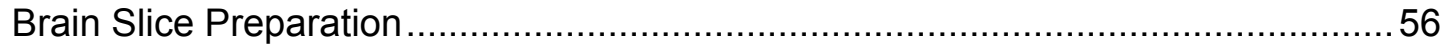

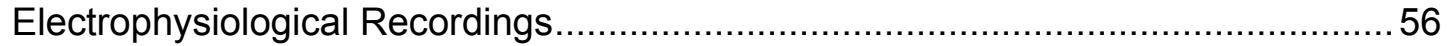

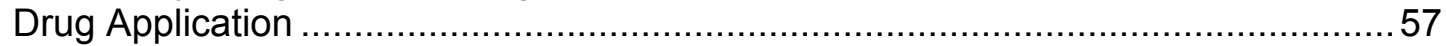

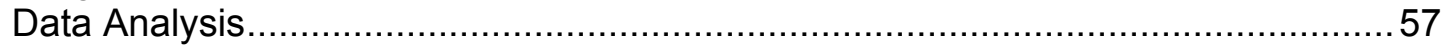

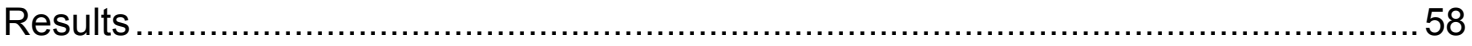

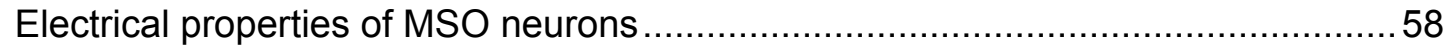

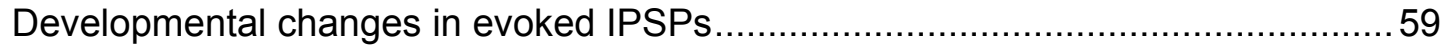

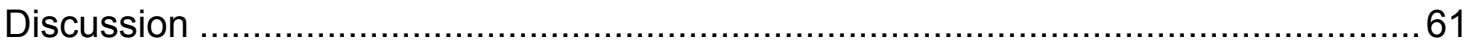

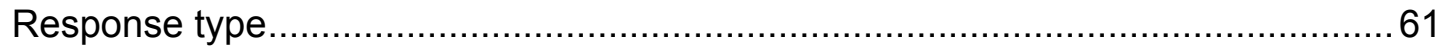

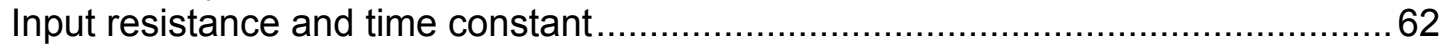

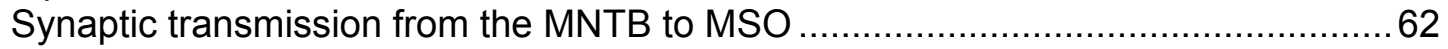

Chapter 4: Characterization of a model MSO neuron to generate simulated auditory

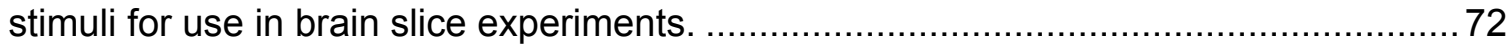

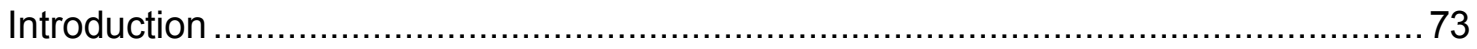

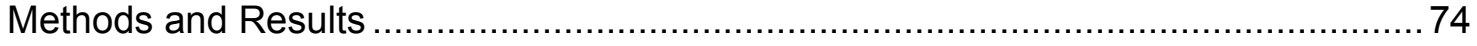

Morphology of MSO Cells-Selection of the Model Cell ..........................................74

Characterization of the Model MSO: Cell-Simulations in NEURON …....................76

Effects of changing the time to peak $(\alpha)$ of the alpha function ............................... 77

Effects of synaptic location on the EPSP shape at the soma ................................ 77

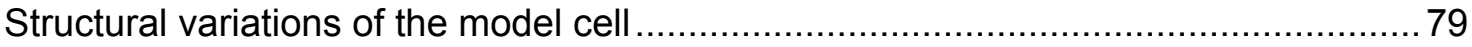

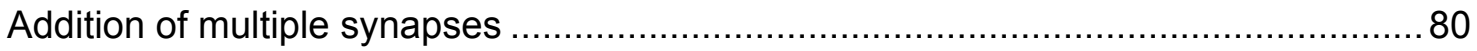


Chapter 5: The roles of voltage gated potassium channels and synaptic inhibition on the coding of ITDs by medial superior olive cells.................................................... 94

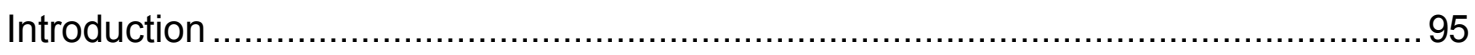

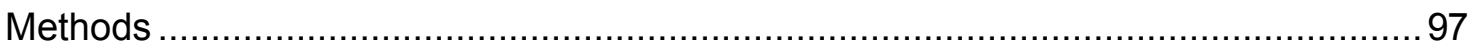

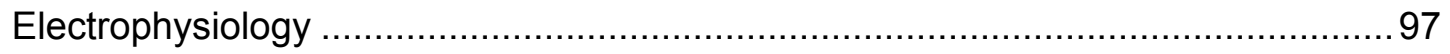

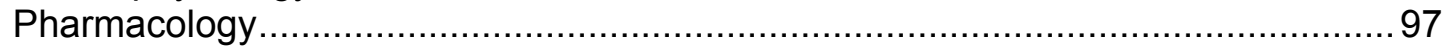

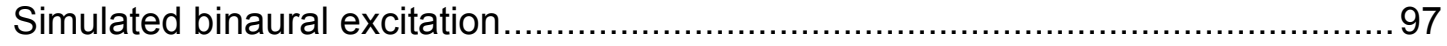

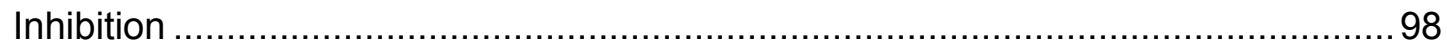

Simulated binaural excitation coupled with activation of inhibitory inputs ................ 99

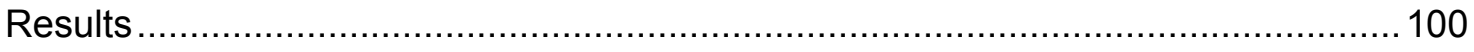

Potassium channels determine the phasic response.......................................... 100

Three types of afterhyperpolarizations follow the action potential in MSO neurons .. 101

Phasic and Tonic cells differ in sensitivity to ITD ............................................. 102

Blockade of $\alpha$-DTX sensitive potassium channels decreased the cell's sensitivity to

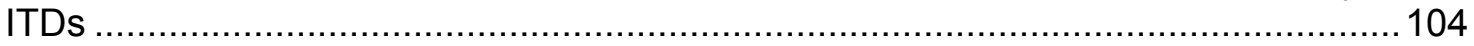

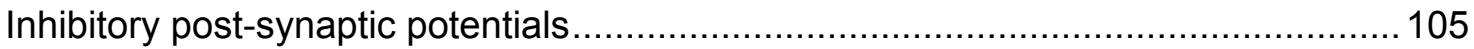
Activation of inhibitory inputs during simulated excitation: effects of lagging inhibition

Simultaneous onset of inhibition and excitation............................................. 107

Activation of inhibitory inputs during simulated excitation: effects of leading inhibition

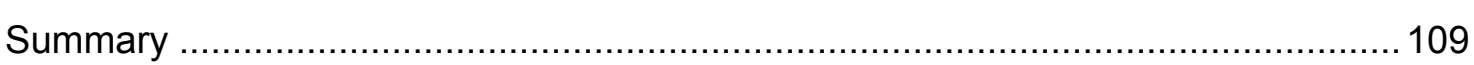

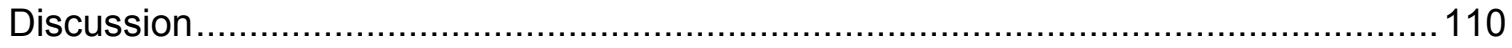

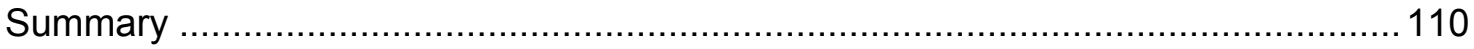

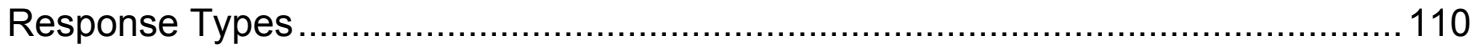

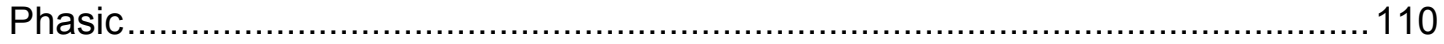

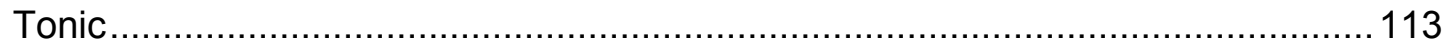

Chapter 6: Suggestions and General Discussion .......................................... 141

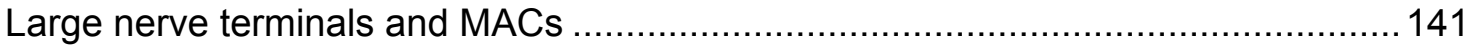

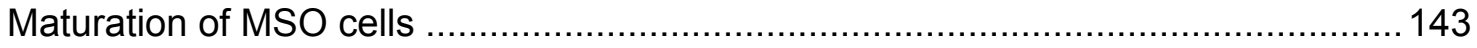

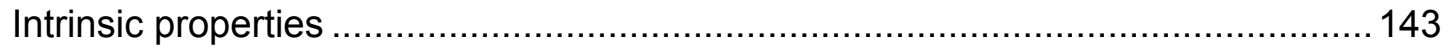

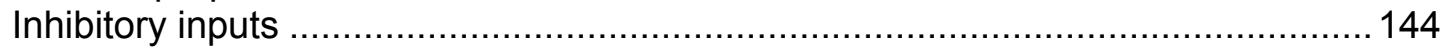

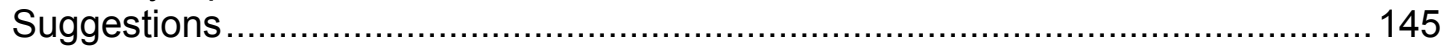

Generation of simulated ITDs; characterization of the model cell........................... 145 
The roles of voltage gated potassium channels and synaptic inhibition on the coding of ITDs by MSO cells. 146

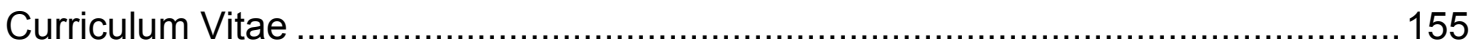

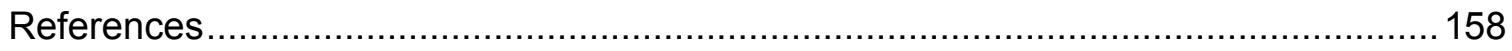




\section{LIST OF FIGURES \\ Chapter One}

Figure 1. Auditory brainstem circuitry

\section{Chapter Two}

Figure 1. The calyx of Held is a large, complex nerve terminal that envelops the postsynaptic neuron and has multiple synaptic sites.

Figure 2. Serial sections indicate that MACs are adjacent to synapses.

Figure 3. The interdigitated spatial relationship of synapses and MACs is revealed in en face views of the flattened presynaptic membrane.

Figure 4. Coated and noncoated vesicles appear fused with the vesicular chain

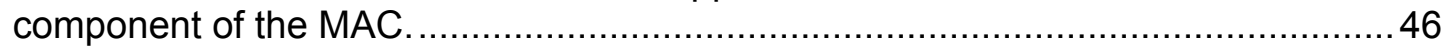

Figure 5. Mitochondria can form more than one MAC and have complex geometry. ...48

Figure 6. Synapses vary in the number of docked vesicles....................................50

Figure 7. Auditory brainstem circuitry...

\section{Chapter Three}

Figure 1. Representative records of membrane properties and firing patterns of MSO

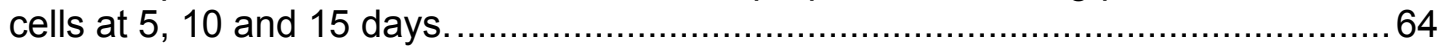

Figure 2. Distribution of cell types during the first 19 days of postnatal life. .................66

Figure 3 . The input resistance of phasic cells and tonic cells decreased during the second week of life.

Figure 4. Mean values of membrane time constant of phasic and tonic cells versus age of the animal.

Figure 5. Mean values of the resting membrane potential at each of the different ages studied

Figure 6. Temporal characteristics of IPSPs versus age.

Figure 7. Plot of average time constant of recovery of IPSPs versus the average membrane time constant.

\section{Chapter Four}

Figure 1. Cameral lucida drawings of ten intracellularly labeled MSO cells

Figure 2. Scatterplot of summed medial versus lateral lengths of thick dendrites. .........86

Figure 3. EPSPs recorded from the soma of the model cell.

Figure 4. The amplitude, time to peak and duration of EPSPs change with synaptic location.

Figure 5. Effects of structural variations of the model cell on EPSPs recorded in the soma.

Figure 6 . Addition of excitatory synapses increases the amplitude of the voltage response in the soma.

Figure 7. EPSPs recorded from the model MSO cell at different simulated ITDs.

\section{Chapter Five}

Figure 1. Semi-schematic of the superior olivary complex of the gerbil.

Figure 2. Stimulation protocol used to study the effects of varying the onset of inhibition during simulated ITD current injections.

Figure 3. Voltage responses to hyperpolarizing and depolarizing current injections. .. 119 
Figure 4. Phasic neurons have steeper ITD curves than tonic neurons.

Figure 5A-E. Graphs of control simulated ITD curves from 40 phasic cells............... 127

Figure 6. Representative ITD curves from tonic neurons....

Figure 7. Phasic neurons have low input resistances and steep ITD curves whereas tonic neurons have high input resistances and shallow ITD curves.

Figure 8. Normalized spike counts in response to simulated ITD for phasic (left) and tonic (right) neurons before and following application of $\alpha$-DTX

Figure 9. The ability of MSO cells to follow MNTB stimulation with IPSPs decline with increasing frequency.

Figure 10. Normalized IPSP amplitude versus number in the train.

Figure 11. Voltage recordings of MSO cells injected with excitatory current alone and conditions with combined excitation and inhibition.

Figure 12. ITD curves of control and lagging inhibition....

Figure 13. Voltage recordings during control and simultaneous onset of inhibition conditions.

Figure 14. Simultaneous onset of inhibitory inputs decrease the normalized spike rate of ITD curves.

Figure 15. Voltage recordings during control and leading inhibition conditions.......... 138

Figure 16. Effects of leading inhibition on the coding of ITDs.

Figure 17. Composite ITD curves for two cells.

\section{Chapter Six}

Figure 1. Integration of excitatory and inhibitory synaptic input in a model cell. 152

Figure 2. Schematic of proposed MSO model. 
Chapter One 


\section{Literature Review}

\section{Introduction: Sound Localization}

Localizing sounds is important to animals for such diverse tasks as enabling them to find mates and be alerted to potential danger. An animal's ability to localize sounds is aided by a large number of complex structural and functional specializations along the auditory pathway. The two salient cues of sound localization in the azimuthal plane are differences in the time of arrival and level of sounds at the two ears. The difference in sound pressure level at the two ears (interaural level difference or ILD) is dependent upon the frequency of the sound. The wavelengths of high frequency sounds are reflected by the head, and creates ILDs. Low frequency sounds have long wavelengths compared to the size of the head (below $1600 \mathrm{~Hz}$ for a human head) are not reflected by the head and are instead localized by interaural temporal delays (ITDs) (Rayleigh 1877; Rayleigh 1907; Thompson 1878). These two interaural cues define the basis for the duplex theory of sound localization (Rayleigh 1907; Masterton and Imig 1984). The duplex theory postulates that ITDs and ILDs are analyzed by separate neural systems: a system for localizing low frequency sounds by ITDs, and a system for localizing high frequency sounds by ILDs.

The minimum detectable movement of a sound source in azimuth yields a measure termed the minimal audible angle. Humans are able to detect differences between sound sources separated by a minimal audible angle of only one degree. Minimal audible angles of only one degree correspond to an ITD of only a few microseconds (Yost 1974) for locations directly in front of the head (Zwislocki and Feldman 1956). The ability of the auditory system to encode differences in ITD at the two ears of only a few microseconds is remarkable given that the element for neural encoding of information, the action potential, is longer than one millisecond in duration. The ability to encode such small temporal differences is accomplished by morphological physiological specializations at very early stages along the auditory pathway. The neural processes that result in the perception of sound localization are complex and are analyzed in several 
areas in the brain. Given the complexity of each of the ILD and ITD systems, my research was focused on the cellular and synaptic influences that enable cells of the lower auditory brainstem to encode the location of a sound sources by means of ITDs.

Calculations of sound source location by means of ITDs are thought to be executed by a constellation of cell groups in the lower auditory brainstem called the superior olivary complex (SOC). A description of the SOC and its role in sound localization is provided below.

\section{Superior Olivary Complex}

Early stages of neural coding of ITDs and ILDs are accomplished by cells of the superior olivary complex (SOC), where significant binaural convergence from both cochlear nuclei initially occurs (Stotler 1953). The SOC is conserved in many species, with strikingly similar arrangements of its cell groups. The SOC is located in the medulla and is composed of three principal nuclei, the medial nucleus of the trapezoid body (MNTB) and the medial and lateral superior olives (MSO and LSO respectively) (Cajal 1909; Morest 1968). The MNTB is the most medial cell group, the LSO the most lateral, and the MSO is situated between the MNTB and LSO. In some species, a prominent nucleus just dorsal to MSO called the superior paraolivary nucleus is found (SPN) (Saldana and Berrebi 2000). Surrounding the main cell groups of the SOC are glycinergic and GABAergic periolivary nuclei, which number up to eleven in some species. A schematic drawing of the afferent connections to the MNTB, MSO and LSO is depicted in Figure 1.

The overall sizes of each of the nuclei of the SOC vary among different species. Small mammals that have a low frequency range of hearing (100$60,000 \mathrm{~Hz}$ ) and utilize low frequency sounds for survival, such as detecting danger, have a well-developed MSO (e.g. gerbils) compared to other mammals similar in size. In contrast, rats have a higher frequency range of hearing, use high frequency calls for communication and have a rudimentary MSO. Also, larger mammals that use low frequency sounds for either detecting prey or for social communication have a very large MSO. Such mammals include humans, 
monkeys, dogs and cats (Moore 2000; Goldberg and Brown 1968; Schwartz 1984).

\section{The LSO encodes ILDs and the MSO encodes ITDs}

The observation of significant bilateral convergence from each $\mathrm{CN}$ to the SOC led to speculation by early anatomists of its role in sound localization (Cajal 1909). The speculation was confirmed by behavioral studies whereby lesions were made above the level of the SOC in the nuclei of lateral lemniscus, inferior colliculus, medial geniculate, or auditory cortex in experimental animals. Following the lesions, the animals' ability to locate sounds in the hemifield on the opposite side of the lesion was lost (Casseday and Neff 1975; Jenkins and Masterton 1982; Thompson and Masterton 1978). In humans, where trapezoid body function is compromised by either stroke or a demyelinating disease, listeners incorrectly report sounds originating in the opposite hemifield to directly in front of them (Furst and Algom 1995; Pratt et al. 1998).

Further experiments that specifically tested physiological responses of the LSO and MSO showed that they had distinct sensitivity to different sound localization cues. Neurons of the LSO alter output spike patterns based upon ILDs of high frequency sounds (Boudreau and Tsuchitani 1968; Goldberg and Brown 1968). LSO neurons are excited by the ipsilateral $C N$ and inhibited by the contralateral $\mathrm{CN}$ via innervation of the MNTB; the MNTB converts the excitatory signal into an inhibitory one and then projects to the LSO.

In contrast to the LSO, MSO neurons increase their firing rates with stimulation of either ear, but firing rates are greatly enhanced by binaural stimulation and are sensitive to changes in ITD (Goldberg and Brown 1968; Spitzer and Semple 1995; Yin and Chan 1990; Yin and Chan 1990). The cellular and synaptic mechanisms that govern the changes in firing rate with ITD are only beginning to be understood. 


\section{Medial Superior Olive}

\section{MSO: Excitatory Afferents}

The MSO integrates excitatory and inhibitory inputs arising from both sides of the brain. The major source of excitatory input to the MSO arises from spherical bushy cells (SBCs) of the anteroventral region of the cochlear nucleus (AVCN) (Stotler 1953; Warr 1972; Cant and Casseday 1986; Osen 1969). SBCs are activated by the large axon terminals of auditory nerve fibers called endbulbs of Held (Sento and Ryugo 1989). Large axon terminals function to reliably transmit presynaptic spike activity through the synaptic junction to the postsynaptic neuron. Therefore, SBC activity resembles auditory nerve fiber activity with very little transformation of temporal patterns in the spike train. Thus, neural input to MSO cells from SBCs strongly resembles that of the auditory nerve.

The first experiments demonstrating bilateral projections from SBCs of the AVCN to the MSO came from lesion studies of the cochlear nucleus (Stotler 1953). Following lesions, cells of the ipsilateral MSO lacked axon terminals on the dendrites oriented towards the lesioned CN (lateral dendrites) where under normal conditions the dendrites were studded with axon terminals. The contralateral MSO also lacked axon terminals on the dendrites directed towards the lesioned cochlear nucleus (medial dendrites), indicating a bilateral projection from the $\mathrm{CN}$ to the MSO of both sides. Other investigations using the lesion technique have come to the same conclusion (Warr 1972; Clark 1969a; Clark 1969b; Perkins 1973a). These studies provide evidence that the MSO is contacted from both $\mathrm{CN}$ and the projections from each $\mathrm{CN}$ innervate the neuron's dendritic pole oriented toward that $\mathrm{CN}$.

MSO cells are contacted by many bouton-type nerve endings innervating the soma and dendrites. Nerve terminals fixed with aldehydes can reveal the excitatory or inhibitory nature of the terminal by the shape of synaptic vesicles (Uchizono 1965; Bodian 1966). Round synaptic vesicles are an indicator that the neurotransmitter contained within the vesicles is excitatory, whereas flattened or pleomorphic vesicles indicate inhibitory neurotransmitter. Most of the terminals 
contacting the MSO cell body and dendrites contain large, round synaptic vesicles (Berrebi and Spirou 1998; Perkins 1973c; Schwartz 1972; Lindsey 1975b; Kiss and Majorossy 1983; Russell and Moore 2002; Smith et al. 1993). More direct evidence that excitatory terminals contact the MSO was obtained by a study where single SBC axons were labeled with horseradish peroxidase (HRP) and the nerve terminals contacting MSO cells were viewed with the electron microscope. The synaptic vesicles contained within the HRP-labeled nerve terminals were large and round indicating that the SBC input to MSO cells is excitatory (Smith et al. 1993). Although morphological studies only provide indirect evidence, many studies indicate that the inputs from SBCs are excitatory.

Evidence for bilateral excitatory innervation to MSO cells has been demonstrated by using brain slices. Excitatory post-synaptic potentials (EPSPs) were evoked in MSO cells when fibers on either side of the MSO were stimulated (Grothe and Sanes 1993; Sanes 1993; Smith 1995). The results of these studies did not identify the cell types being activated in the $\mathrm{CN}$ but demonstrated bilateral innervation to the MSO from excitatory inputs.

MSO: Inhibitory Afferents

In addition to the excitatory input from the $\mathrm{CN}$, MSO cells are substantially innervated by inhibitory inputs, as evidenced from immunocytochemistry, electron microscopy and tract-tracing studies. Axon terminals, immunopositive for the inhibitory neurotransmitters glycine and GABA, surround MSO cell bodies (Roberts and Ribak 1987; Glendenning and Baker 1988; Helfert et al. 1989; Spirou and Berrebi 1996). The sources of inhibitory input to MSO cells lie from within the SOC. Tract tracing and intracellular labeling studies indicate that, in addition to a strong input to the LSO, collaterals of MNTB axons make synaptic connections ending on MSO cells (Adams and Mugnaini 1990; Banks and Smith 1992; Kuwabara and Zook 1992; Smith et al. 1998). Injection of the retrograde tracer, HRP, into the MSO also revealed another inhibitory input from the lateral nucleus of the trapezoid body (LNTB) (Cant and Hyson 1992a). Both the MNTB and posteroventral LNTB (pvLNTB) label strongly for the inhibitory neurotransmitter glycine (Wenthold et al. 1987; Peyret et al. 1987; Helfert et al. 
1989; Adams and Mugnaini 1990; Henkel and Brunso-Bechtold 1995; Ostapoff et al. 1997; Spirou and Berrebi 1997). Other cells of the LNTB show moderate immunoreactivity (main or mLNTB) or are immunonegative (hilus or hLNTB) to glycine. In addition to glycinergic cells, the mLNTB also contains a population of neurons that are GABAergic (Spirou and Berrebi 1997). Assuming that immunoreactivity is indicative of neurotransmitter phenotype, then the LNTB contains a mixture of both glycinergic and GABAergic cells that could work to inhibit cells of the MSO.

Further evidence for inhibitory inputs to the MSO are shown in electron micrographs of MSO cells. Many of the axon terminals make synaptic contact onto the cell body and dendrites contain vesicles that are flattened or pleomorphic in shape (Perkins 1973b; Schwartz 1972; Lindsey 1975a; Kiss and Majorossy 1983; Russell and Moore 2002; Smith et al. 1993), indicating that the vesicles contained in those terminals contain inhibitory neurotransmitter.

Stimulation of both the medial and lateral fibers leading to the MSO in brain slices, while pharmacologically blocking excitatory neurotransmission, reveals the presence of bilaterally activated inhibitory inputs (Grothe and Sanes 1993). Based upon the previously mentioned morphological studies, these investigators deduced that the MNTB provides contralaterally activated inhibition and the LNTB provide ipsilaterally activated inhibitory inputs.

\section{MSO: Efferents}

The efferent projections of MSO cells provide further evidence for their pivotal role in sound localization. Axons of MSO neurons project ipsilaterally to the central nucleus of the inferior colliculus and likely convey ITD sensitivity to target neurons (Adams 1979; Brunso-Bechtold et al. 1981; Elverland 1977; Henkel and Spangler 1983; Roth et al. 1978; Spangler et al. 1985; Beyerl 1978; Glendenning and Masterton 1983; Jones and Cullen 1979; Kudo et al. 1990; Okoyama et al. 1995; Oliver et al. 1995). Some axon terminals originating from the MSO also innervate cells of the ventral nucleus of the lateral lemniscus (VNLL)(Glendenning et al. 1981; Henkel and Spangler 1983) and dorsal nucleus of the lateral lemniscus (DNLL) (Glendenning and Masterton 1983). The MSO 
also innervates the contralateral inferior colliculus (IC), but the projection is only a small component of the efferent projection (Brunso-Bechtold et al. 1981; Nordeen et al. 1983; Roth et al. 1978; Adams 1979; Glendenning et al. 1981; Henkel and Spangler 1983). In most mammals, MSO cells are immunonegative to the neurotransmitters, glycine, GABA and the synthesizing enzyme for GABA, glutamic acid decarboxylase (GAD). The evidence is indirect, but suggests that the primary projection of the MSO is an ipsilateral, excitatory projection to the IC.

In summary, cells of the MSO are excited bilaterally from SBCs of each cochlear nucleus and are inhibited by contralaterally activated cells of the MNTB and from ipsilaterally activated cells of the LNTB. The MNTB, LNTB and SBCs are all activated by unusually large synaptic nerve terminals. These terminals are thought to act in conjunction with their postsynaptic target cells to provide secure excitatory and inhibitory timing information about the stimulus of the sound to MSO cells. A review of the terminals found in the lower auditory brainstem is provided below.

\section{Large nerve terminals are a hallmark of the lower auditory system}

One of the striking features of the central auditory system is the size of the nerve terminals contacting many neurons of the lower auditory brainstem. Activation of the inhibitory cells that project to the MSO occurs via globular bushy cells (GBCs) of the cochlear nucleus. GBCs, mostly located in the ventral root of the $\mathrm{CN}$, innervate cells of the ipsilateral LNTB and project across the midline to the contralateral MNTB. GBCs share similarities with SBCs in that they are contacted by synaptic terminals called end-bulbs of Held (Cant and Morest 1979; Ryugo and Sento 1991; Ryugo et al. 1996; Ryugo et al. 1997). End-bulbs contacting SBCs are larger than the terminals innervating GBCs. When viewed under the electron microscope, end-bulbs have an abundance of round synaptic vesicles (Gentschev and Sotelo 1973; Sotelo et al. 1976; Neises et al. 1982; Wang and Kaczmarek 1998) and more than 150 synaptic junctions (also called active zones) as estimated by serial section electron microscopy of complete reconstructions of end-bulbs (Nicol and Walmsley 2002). The large number of synaptic contacts in a given nerve terminal indicate that a large number of 
synapses are active for every incoming action potential and point to the mechanism by which signals are faithfully transmitted from one neural station to the next in the pathway.

Cells of the MNTB are contacted by a single, large calyceal terminal called the calyx of Held (Held 1893; Cajal 1909). Cells of the MNTB are innervated by only one calyx of Held per cell, but a single GBC axon can split and give rise to two calyces on two separate cells (Spirou et al. 1990; Smith et al. 1991; Kuwabara et al. 1991). The calyx of Held is perhaps the largest nerve terminal in the central nervous system and in some electron micrographs envelopes more than half of the MNTB somal surface area (Smith et al. 1991). Calyces take on a claw-like shape, which have finger-like processes, called stalks. Stalks end in swellings, which are linked to smaller swellings by thin 'necks' (Rowland et al. 2000).

The GBC input to cells of the pvLNTB take on a similar form to calyces, but, rather than a single nerve terminal, large bouton endings link together into complex pericellular nests around pvLNTB cells (Spirou and Berrebi 1996). We recently examined the large terminals contacting cells of the LNTB (Spirou et al. 1998). Individual electron micrographs showed that over $18 \%$ of the nerve terminals contacting pvLNTB cells were greater than 10 microns in length, compared to only $3 \%$ contacting cells in the main region of the LNTB, illustrating the large size of these terminals compared to terminals found in other nuclei of the brain (Spirou et al. 1998). The input from the large nerve terminals is excitatory as indicated by the overwhelming majority of round synaptic vesicles found in the terminals of the MNTB and LNTB (MNTB:Lenn and Reese 1966; Rowland et al. 2000; LNTB:Smith et al. 1991; Spirou et al. 1998) which do not immunoreact to antibodies against glycine and GABA (Spirou et al. 1998).

\section{Unique organelle arrangements are found in large nerve terminals}

We recently made electron microscopic observations in the terminals contacting pvLNTB cells (Spirou et al. 1998) of an unusual assemblage of organelles, originally described in nerve terminals of the spinal cord (Gray 1963) and later in end-bulbs contacting SBCs (Cant and Morest 1979). We named this 
unique assembly of organelles the mitochondria-associated adherens complex (MAC). The central feature of a MAC is a mitochondrion positioned close to (about $250 \mathrm{~nm}$ ) a contact point of the presynaptic membrane. The contact point of the presynaptic membrane is a set of symmetrical pre- and postsynaptic electron dense plaques, thought to hold those two membranes together, typically called punctum adherens. (For further description of MACs see Spirou et al., 1998). We observed in several electron micrographs that MACs were positioned nearby synaptic vesicle docking sites called active zones. The observations of MACs in the large nerve terminals contacting pvLNTB cells and their relative proximity to active zones prompted our investigation to determine the existence, frequency and spatial relationship between MACs and synapses in calyces of Held (described in detail in Chapter 2).

\section{Physiological relevance of large nerve terminals}

The morphological features of the calyx and large nerve terminals of the LNTB suggest that calyces are able to provide extremely well timed, high fidelity inhibitory input to their postsynaptic targets. The precise timing of inputs is assisted by extremely short synaptic delays (Forsythe and Barnes-Davies 1993a) and low temporal variability in release of neurotransmitter by synaptic vesicles. The variability in vesicle release is known as synaptic jitter. When an action potential invades the calyx, enough neurotransmitter is released onto the postsynaptic cell to cause the cell to reach threshold and generate an action potential. This one-to-one pre- to postsynaptic spiking was suggested by investigators conducting morphological studies (Morest 1973) and confirmed by electrophysiologists (Forsythe and Barnes-Davies 1993a; Borst et al. 1995). The calyx has also been shown to follow high rates of repetitive stimulation (Guinan, Jr. and Li 1990; Wang and Kaczmarek 1998). Despite the vast literature on the physiology of the calyx/MNTB synapse, a defined role in the processing of ITDs has not been made. 


\section{MSO: In vivo Physiology Experiments}

The physiological responses of MSO cells to binaural stimulation have long been considered to act in accordance with a model proposed by Jeffress (Jeffress 1948). The Jeffress model states that a binaural comparator nucleus encodes ITDs and that neural delay lines to the nucleus offset the delays imposed by the location of the sound source. The Jeffress model also assumes that input from each ear is below action potential threshold of the binaural comparator cell and only sums to generate an action potential when input from both sides arrive in coincidence. If the sound moves from the original position, the input from each side becomes temporally mismatched and activity decreases in that cell, whereas in other cells activity increases. If the ITDs yielding maximal activity are mapped across the axis of the cell column, then the location of active cells becomes the code for sound localization.

The first physiological study that pointed to the MSO as the anatomical substrate of the Jeffress model was reported by Moushegian (Moushegian et al. 1964). In these experiments, the activity rate of MSO cells increased dramatically under binaural stimulation, as expected for cells bilaterally innervated from each cochlear nucleus. The first experiments revealing sensitivity of the MSO cells to ITDs were single unit recordings made in dogs (Goldberg and Brown 1969). The spike rate of MSO cells increased under binaural stimulation and responded vigorously when the sounds at the two ears were presented over a small range of ITDs. At certain ITDs, however, the spike rate fell below that seen for either the monaural and spontaneous rates. Goldberg and Brown proposed that inhibition caused the decrease in spike rate at 'unfavorable' ITDs.

In the experiments by Goldberg and Brown the maximum spike rate in the response (called the peak ITD) occurred when the contralateral sound was presented prior to the ipsilateral sound (Goldberg and Brown 1968) indicating that the MSO is biased towards contralateral sounds (Yin and Chan 1990; Spitzer and Semple 1995). One of the assumptions of the Jeffress model is that the maximum spike rate encodes the location of the sound source and therefore 
the spike output of each cell represents a discrete position on the azimuth (Jeffress 1948). The peak ITD however, often occurred at an ITD longer than the animal could experience based upon the size of the head. Therefore, the peak ITD is outside the physiological range and away from midline. The paradox between peak ITDs and the physiological range of ITD has been addressed in recent studies of ITD sensitive neurons in the IC (McAlpine et al. 2001) and MSO (Brand et al. 2002). The studies of McAlpine and colleagues suggest that the peak ITD is unimportant compared to the position on the ITD curve that generates the greatest change in spike rate. They showed that irrespective of the best frequency of the neuron, the greatest change in neural discharge rates occurred at midline (0 ITD). The results of the McAlpine study suggest that when the sound source is directly in front of the head and generates no ITD, the overall activity level of the MSO on both sides operates at $50 \%$ of the maximum spike rate. When the sound moves away from midline, activity increases in the contralateral MSO, whereas it decreases in the ipsilateral MSO. These data were used to explain the increased resolution of sound source discrimination at midline ITDs.

The mechanism of the shifting of the peak ITDs away from midline ITDs so that the largest slope of the ITD fell around midline ITDs was recently hypothesized to result from a precisely timed inhibitory input (Brand et al. 2002). Responses of ITD sensitive neurons were studied before and following application of iontophoretically applied strychnine (Brand et al. 2002). Blockage of glycine receptors by strychnine increased the discharge rate, and, interestingly, the peak ITDs shifted from outside of the physiological range to values centered on zero. Their neural simulations suggested that peak ITDs are shifted outside the physiological range by a precisely timed inhibitory input that arrives $200 \mu$ secs prior to the excitatory input. The results by Brand et al. are still in question because the physiological experiments were performed on only five cells and the proposed inhibitory mechanism was supported only through neural simulations of ITD. 
The arrival time of inhibitory postsynaptic potentials (IPSPs) relative to excitatory postsynaptic potentials (EPSPs) in the MSO is not known, but the possibility that IPSPs arrive prior to excitation, despite a delay imposed by another neural station, is plausible. This can be accomplished in part by the fast conduction velocity and extremely short synaptic delays recorded from the calyx to MNTB synapse (Taschenberger et al., 2000S) causing the IPSPs to arrive prior to EPSPs in the MSO. The timing of inhibition to the MSO could have profound effects on whether EPSPs sum to generate action potentials and therefore shape ITD curves. Studying the temporal aspects of inhibition in vivo is very difficult because the temporal activation of inhibitory inputs cannot be experimentally manipulated. Effects of temporal variations of inhibitory inputs are best studied by use of the brain slice preparation or from computer simulations. We aimed to study the effects of inhibitory inputs on ITD coding by using a brain slice preparation in young gerbils.

\section{MSO: Brain Slice Electrophysiology}

MSO neurons, due their compact arrangement and generation of large potentials by acoustic stimuli, are difficult to isolate in single unit recordings. Determining the mechanisms responsible for the shaping of ITD curves is made easier by the use of brain slice electrophysiology as the technique allows for direct access to the cells of interest.

Recordings made from principal cells of the MSO in brain slices are very similar to the so-called type II responses that have been recorded from cells located in the anteroventral cochlear nucleus (AVCN) (Oertel 1983; Wu and Oertel 1984), MNTB (Brew and Forsythe 1995; Banks and Smith 1992) and nucleus laminaris, the avian homologue of the MSO (Zhang and Trussell 1994; Manis and Marx 1991). Type II responses are characterized by one or two action potentials at the start of a sustained, depolarizing current injection followed by a steady state voltage without the generation of action potentials.

The lack of spikes after stimulus onset in type II cells is the result of an increased conductance to potassium ions at depolarized membrane potentials, as was first studied by using voltage clamp by Manis and Marx (Manis and Marx 
1991). The increased conductance to potassium ions can be blocked by tetraethylammonium (TEA), 4-aminopyridine (4-AP), and dendrotoxin (DTX) and switches the response of the cell from a single spike at current onset (phasic) to a cell that generates repetitive action potentials (tonic)(Manis and Marx 1991; Banks and Smith 1992; Forsythe and Barnes-Davies 1993b; Smith 1995; Brew and Forsythe 1995).

\section{Potassium channels}

Potassium channels were among the first channels investigated by use of the voltage clamp technique (Hodgkin et al. 1949) and affect the shape, threshold, and firing cadence of action potentials (Hille 1992; Golowasch et al. 1992; Ma and Koester 1996). There are four main families of voltage-gated potassium channels, named Kv 1, 2, 3 and 4. Each family is distinct in its voltage dependence and kinetic properties (Jan and Jan 1997).

At least two potassium conductances shape the intrinsic responses of neurons of the auditory brainstem. One of the conductances is activated at membrane potentials near rest and is termed a "low threshold" potassium current. The other is a current that is activated at potentials near action potential threshold $(-20 \mathrm{mV})$ and is termed a "high threshold" potassium current.

There is a suggestion from a study using immunocytochemistry that Kv1.1 and 1.2 are expressed by cells in the murine MSO (Grigg et al. 2000). The investigators did not distinguish between the MSO and LSO and analyses of their immunoreactivity were combined. The MNTB was examined alone, and had the highest immunoreactivity for $\mathrm{Kv} 1.1$ and 1.2 channels among all the nuclei studied. Other immunocytochemical data indicate that the MNTB expresses Kv1.1, 1.2, and 1.6 (Dodson et al. 2002a). The similar response patterns to intracellular current injection exhibited by MNTB and MSO neurons indicate that they may express similar types of $\mathrm{Kv}$ channels. However, there are no immunocytochemical studies focused on the MSO to indicate the kinds of voltage-gated potassium channel proteins that are expressed by these cells.

A pharmacological approach using toxins that block specific ion channels can provide clues to the types of channels found in particular cells. Studies from 
cloned potassium channels indicate that toxins isolated from the African green mamba snake (Dendroaspis augusticeps) selectively block specific members of the Kv 1 family. One of these toxins, named $\alpha$-dendrotoxin ( $\alpha$-DTX), selectively blocks Kv1.1, 1.2, and 1.6 channels in the nanomolar range (Harvey and Karlsson 1980; Harvey and Karlsson 1982; Grissmer et al. 1994; Ferroni et al. 1992; Werkman et al. 1992; Swanson et al. 1990). When $\alpha$-DTX was superfused into the bath during current-clamp recordings of MNTB cells, cells confirmed by immunocytochemistry to contain $\mathrm{Kv1} 1,1.2$ and 1.6 channels, the phasic response was abolished and tonic responses were initiated (Dodson et al. 2002a). In the same study, when potassium currents were isolated under voltage-clamp, $\alpha$-DTX blocked potassium currents that began activation at a very low voltage (slightly more positive than $-70 \mathrm{mV}$ ) and only slightly inactivates. The current, referred to as a "low threshold" potassium current $\left(I_{K L T}\right)$, is similar to currents recorded from isolated bushy cells VCN (Manis and Marx 1991), octopus cells of the CN (Bal and Oertel 2001; Ferragamo and Oertel 2002), and onset cells of the inferior colliculus (Sivaramakrishnan and Oliver 2001).

In addition to low threshold potassium channels, some neurons of the auditory brainstem express potassium channels that are strongly activated at depolarized voltages (about $-20 \mathrm{mV}$ ), called "high threshold" potassium channels (Rathouz and Trussell 1998; Perney and Kaczmarek 1997; Parameshwaran et al. 2001). Studies indicate that MNTB neurons contain a potassium channel, specifically $\mathrm{Kv} 3.1$, distinguished by a high activation threshold and lack of inactivation during high frequency stimulation (Wang et al. 1998). Activation of high threshold potassium currents provides a rapid repolarization during action potentials, thus making action potentials more brief and allowing for high frequency spike generation. The importance of $\mathrm{Kv} 3.1$ channels in high frequency firing was demonstrated by block of the high threshold current with TEA. When TEA was added to the bath, the ability of the cell to follow frequencies beyond $200 \mathrm{~Hz}$ was eliminated (Wang et al. 1998). To date, there are no studies that indicate the presence of Kv3 channels in MSO cells.

A type of potassium channel that carries potassium ions and is activated 
by calcium $\left(\mathrm{K}_{(\mathrm{Ca})}\right)$ can effect the hyperpolarization phase of the action potential (Cangiano et al. 2002). Recently, the Slack gene (sequence like a Ca-activated $\mathrm{K}$ channel) was identified by using immunocytochemistry in neurons of the MNTB (Bhattacharjee et al. 2002). The presence of Slack or other $\mathrm{K}_{(\mathrm{Ca})}$ channels in other nuclei of the auditory brainstem is not known. 


\section{Objectives}

\section{Study 1 (Calyx)}

Large nerve terminals are a hallmark of lower auditory brainstem circuitry. We aimed to further describe the morphology of the giant nerve terminal, the calyx of Held. We also report on an arrangement of organelles, consisting of a mitochondrion located near the presynaptic membrane, tethered via filaments to a punctum adherens, which we named the mitochondria-associated adherens complex (MAC)(Spirou et al. 1998). The MAC was first described in nerve terminals of the spinal cord (Gray 1963), and, in the auditory system, was noted in large terminals of auditory nerve fibers (Cant and Morest 1979; Tolbert and Morest 1982a). We noted the adjacency of MACs to regions of synaptic vesicle binding sites, called active zones, implying a role in synaptic transmission. Previous investigators had not published any observations on the existence of MACs in calyx of Held terminals.

We aimed to determine the spatial relationship between MACs and active zones in the calyx of Held. We provide hypotheses that a close spatial relationship between MACs and synapses would aid calyces of Held in conveyance of a well-timed, highly precise inhibitory input to cells of the MSO.

\section{Study 2 (Maturation of MSO cells)}

The basic elements of the circuitry of the lower auditory brainstem that compute sound source location by ITDs have been identified, but the physiological mechanisms employed by those elements are poorly understood. Electrophysiological recordings from brain slice preparations from young animals are easy to obtain and offer direct means to study intrinsic ionic conductances. Recordings from older animals are not yet possible, due to the high density of myelinated axons in and around the MSO, thus limiting visualization of neurons. We aimed to determine the roles of potassium channels and inhibitory inputs in coincidence detection. Before performing these experiments, we studied the maturation of the membrane electrical properties to determine if a physiologically 
stable age range exists. The development of the electrophysiological properties of MSO cells has not been reported.

\section{Study 3 (Role of potassium channels and inhibitory inputs in ITD coding by MSO cells)}

Whether Kv1 channels or $\alpha$-DTX sensitive channels exist in the MSO is currently unknown. Since there are currently no studies demonstrating the contribution of potassium channels to the coding of ITDs, we aimed to determine the contribution of potassium channels to the coding of ITDs, by their blockade with $\alpha$-DTX.

A recent study suggested, using a computer model of MSO cells, that inhibitory inputs that arrive prior to excitatory inputs shape the response curve of MSO cells, such that the steepest portion of the ITD curve centers on zero ITD (Brand et al. 2002). These results imply that the timing and synaptic weight of inhibitory inputs shape the MSO response. Inhibitory inputs could work in several ways. An IPSP that arrives after the EPSP but before the EPSP peaks, could shape the EPSP by decreasing its amplitude. If the IPSP arrives at the peak or just following the peak of the EPSP, the falling phase of the EPSP will be shortened. Both of these situations decrease the effectiveness of EPSPs, so that only nearly coincident EPSPs will sum to generate action potentials. Although the MNTB is thought to provide a well-timed glycinergic input to MSO cells, other sources may contribute less precise inhibitory inputs to the MSO, mainly from the GABAergic cells of the main portion of the LNTB. We aimed to study the influences of differences in the variations in the arrival time of IPSPs in the MSO. 


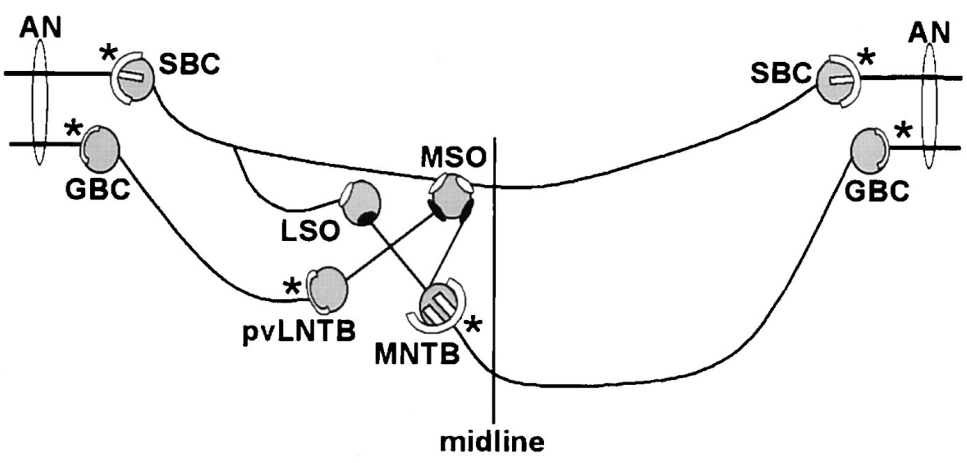

Figure 1. Auditory brainstem circuitry.

Auditory nerve (AN) fibers end in large nerve terminals onto spherical (SBC) and globular (GBC) bushy cells of the cochlear nucleus. GBCs project ipsilaterally, via modified end-bulbs, to cells of the posteroventral lateral nucleus of the trapezoid body (pvLNTB) and contralaterally, via calyces, to cells of the medial nucleus of the trapezoid body (MNTB). Cells of the medial superior olive (MSO) and lateral superior olive (LSO) compare bilateral excitatory (from spherical bushy cells) and inhibitory (from LNTB and MNTB cells) inputs to assign a location to a sound source in space. 
Chapter Two: Specialized Synapse-Associated Structures within the Calyx of Held

This work is published in the Journal of Neuroscience 2000 v.20 (24): 9135-9144. 


\begin{abstract}
The calyx of Held exhibits fast glutamatergic neurotransmission at high rates with low temporal jitter and has adapted specialized synaptic mechanisms to support its functional demands. We report the presence in calyces of an atypical arrangement of subcellular organelles, called the mitochondriaassociated adherens complex (MAC). We demonstrate that MACs are located adjacent to synapses and contain membranous elements linked with coated and uncoated vesicles. Mitochondria that form MACs have more complex geometries than other mitochondria within the calyx and can extend between clusters of synapses. We estimate that the calyx contains 1600 MACs, 2400 synapses, and 6200 readily releasable vesicles. We also identify synaptic vesicle endocytotic regions close to MACs and synapse and hypothesize that calyces are composed of multiple activity modules, each containing machinery for vesicle release and recycling.
\end{abstract}


Introduction

The calyx of Held is one of the largest nerve terminals in the CNS (Held 1893). Along with large and modified end-bulbs of Held delivered by auditory nerve fibers onto cochlear nucleus neurons (Cant and Morest 1979; Tolbert and Morest 1982b) (Fekete et al. 1984), these complex nerve terminals are key elements in brainstem circuitry that subserves sound localization (Morest 1968). Spontaneous activity (generated in the absence of sound) can exceed 100 spikes/sec at the calyx terminal, and sound-driven activity at the most sensitive frequency of the calyceal neuron can approach 600 spikes/sec (Spirou et al. 1990). Temporal synchrony of calyceal neurons to a preferred phase of a low-frequency sound exceeds that found in the auditory nerve, and it can entrain (fire on every cycle of the stimulus sinusoid) at rates approaching $1 \mathrm{kHz}$ (Joris et al. 1994a; Joris et al. 1994b).

High activity rates place demands on endocytotic mechanisms to maintain a pool of releasable vesicles. Some neurons, such as retinal bipolar cells, possess specialized structures called synaptic ribbons that are involved in vesicle trafficking, and therefore have unique mechanisms to solve their demands for synaptic activity (von Gersdorff and Matthews 1999). It is plausible that the calyceal terminal, because of its extremely high spike rate and the temporal precision required to accomplish its task of sound localization, also uses unique structures and mechanisms.

A noteworthy arrangement of organelles, consisting of a mitochondrion located near the presynaptic membrane and tethered via filaments to a punctum adherens, was first described in nerve terminals of the spinal cord (Gray 1963). In the auditory system, this structure was noted in large and modified end-bulbs of auditory nerve fibers (Cant and Morest 1979; Tolbert and Morest 1982a). More recently, we described this structure, which we named the mitochondriaassociated adherens complex (MAC), in large collateral terminals of calyceal axons that are found in the superior olivary complex (Spirou et al. 1998). Given 
the diversity of cellular elements that comprise the MAC, it could perform various functions in the nerve terminal, including calcium buffering, supplying energy for synaptic vesicle fusion and recycling, processing recycled vesicle membrane, and cell adhesion. The presence of MACs in other large terminals of the auditory brainstem prompted our investigation of their presence in calyceal terminals and a more detailed examination of their structure and relationship to synapses. In this report, we reveal an intertwined spatial arrangement of MACs and synaptic structures and offer several hypotheses about possible functions of the structural components of the MAC in synaptic transmission. 
Materials and Methods

Five adult cats were used in this study. Three cats were processed for electron microscopy and two cats were processed for PEP-19 immunocytochemistry. All animals were deeply anesthetized by an intramuscular injection of xylazine $(2 \mathrm{mg} / \mathrm{kg}$ ) and ketamine $(10 \mathrm{mg} / \mathrm{kg})$, followed by a pentobarbital injection (40 mg/kg, i.v.), before transcardial perfusion. The protocol used for PEP-19 immunocytochemistry is described elsewhere (Berrebi and Mugnaini 1991; Spirou and Berrebi 1996; Berrebi and Spirou 1998) and is summarized briefly here.

Immunocytochemistry. Two animals were perfused transcardially with $0.9 \%$ saline solution, followed by $1 \mathrm{I}$ of fixative $(4 \%$ formaldehyde, $0.5 \%$ zinc dichromate, $0.1 \%$ glutaraldehyde in $0.75 \%$ saline, $\mathrm{pH} 4.8$ ), then by 1 I of the same fixative with glutaraldehyde removed. Brains were dissected after $1 \mathrm{hr}$ and immersed in $30 \%$ sucrose in saline for cryoprotection. After cryoprotection, frozen sections taken at 25-40 $\mu \mathrm{m}$ thickness were immersed in blocking and detergent solution for $1 \mathrm{hr}(5 \%$ normal donkey serum, $0.5 \%$ Triton X-100 in $0.5 \mathrm{M}$ Tris buffer). Sections were incubated in rabbit polyclonal antiserum to PEP-19 protein (diluted to $1: 2000$ in $1 \%$ normal donkey serum and $0.1 \%$ Triton $X-100$ ), which was revealed by using the standard Elite avidin-biotin peroxidase method (Vector Laboratories, Burlingame, CA) or the peroxidase/anti-peroxidase method (Sternberger 1979).

Electron microscopy. The animals used for electron microscopy were perfused transcardially with an initial calcium-free Ringer's solution followed by a mixture of $2 \%$ paraformaldehyde and $2.5 \%$ glutaraldehyde in $0.1 \mathrm{M}$ phosphate buffer. The brainstem was cut into $150 \mu \mathrm{m}$ sections in the coronal plane, post-fixed with osmium tetroxide $(0.5 \%)$, and stained en bloc with aqueous uranyl acetate $(2 \%)$, dehydrated, and flat-embedded in Epon. Tissue containing the medial nucleus of the trapezoid body (MNTB), which is innervated by calyces of Held, was reembedded and trimmed for cutting ultrathin sections $(60-70 \mathrm{~nm})$. Sequential 
sections were collected and stained with $0.5 \%$ uranyl acetate and $3 \%$ lead citrate using an automatic grid stainer (Leica, Nussloch, Germany).

Construction of en face diagrams. Individual calyces of Held were tracked in serial ultrathin sections and photographed by using a Jeol 1010 electron microscope at $80 \mathrm{kV}$ with magnifications of 4,000-20,000x. To create en face diagrams, presynaptic membranes and mitochondria were traced from electron micrographs onto transparencies and coded as either synaptic junctions or punctum adherens. Marks for registration of serial sections were added to the tracing by visual determination of the best alignment of all mitochondria on adjacent sections. Synapses were identified as clusters of synaptic vesicles directly associated with dense projections of the presynaptic membrane. Punctum adherens were identified by symmetrically distributed membrane densities without association of synaptic vesicles. Tracings of the coded presynaptic membrane were scanned into a computer and measured using $\mathrm{NIH}$ Image. Total surface areas of synaptic junctions and puncta adherentia were calculated by summing their lengths on individual sections and by multiplying by the section thickness. En face diagrams were created by mapping the location of synapses and punctum adherens relative to register marks (as measured in $\mathrm{NIH}$ Image) using graphing software (Excel, Microsoft). The locations of the vesicular chain or mitochondrial plaque of each MAC were plotted on the en face diagram by placing a symbol over its corresponding punctum adherens. Distances from the edges of the vesicular chain or mitochondrial plaque to the nearest synapse were measured on the en face diagram by using NIH Image. Data are reported as mean \pm SD unless indicated otherwise.

Mitochondria morphology. To create three-dimensional models of mitochondria, electron micrographs were scanned and imported into software for alignment of serial sections (Align, Kristen Harris), and outlines of the mitochondria were marked using software (Trace, Kristen Harris; synapses.bu.edu). Virtual reality modeling language files were created (Trace) and viewed with a threedimensional graphics program (Amapi3D, TGS). 
Results

\section{The calyx of Held is organized into distinct segments}

The calyx of Held is a complex nerve terminal composed of numerous branches linked to swellings of various sizes that, in composite, envelope the postsynaptic cell body. A camera lucida drawing of a calyx and a photomicrograph through a single focal plane (Fig. $1 A$, inset) are shown in Figure $1 A$. Leading to the calyx is a large axon, 5-10 $\mu \mathrm{m}$ diameter in cats, which at the base of the terminal branches into two to four thick stalks (each 3-5 $\mu \mathrm{m}$ diameter; labeled st in Fig. $1 A, B)$ that extend along the postsynaptic cell body surface to the opposite pole of the cell (Fig. 1B). These stalks usually terminate abruptly, but give rise along their distance to thin processes termed necks (Lenn and Reese 1966) (labeled $n$ in Fig. $1 A, E$ ), which connect in series to large and small swellings (labeled $s w$ in Fig. $1 A, E)$. The necks have varying lengths $(2.83 \pm 2.0 \mu \mathrm{m} ; n=70)$ but a relatively consistent diameter of $\sim 1 \mu \mathrm{m}(0.99 \pm 0.42 \mu \mathrm{m} ; n=70)$.

Synaptic junctions are found in the calyceal stalks and swellings. Numerous zones containing synapses alternate along the entire length of the stalks with regions where the presynaptic and postsynaptic membranes separate (Fig. 1B, asterisks; boxed area amplified in $D$ ). Synaptic junctions are also formed with somatic appendages (labeled sa in Fig. 1B) that extend away from the cell body along the sides and end of each stalk. Synaptic junctions are rarely found on the side of the stalk that faces away from the postsynaptic cell body. That side of the stalk is usually apposed to glial processes and myelinated axons. The same arrangement of synapses is found in large and small swellings (data not shown). Necks are not in apposition to the postsynaptic surface, contain neurofilaments, are unmyelinated, and lack synaptic junctions (Fig. 1E).

Regions of apposition of the presynaptic and postsynaptic membranes contain not only synaptic junctions but also specialized organelle assemblies that we have named the MAC (Fig. 1C) (Spirou et al. 1998). The key elements of this 
structure are a punctum adherens $(p a)$, thought to attach the membranes of two cells, tethered via filaments ( $f$ ) that extend $\sim 180 \mathrm{~nm}(179 \pm 12.7 \mathrm{~nm} ; n=26)$ away from the membrane to a dense plaque called the mitochondrial plaque $(m p)$, which is oriented parallel to the membrane and subjacent to a mitochondrion. The mitochondrial surface is typically flattened where it faces the mitochondrial plaque, and its cristae are oriented perpendicular to its flattened surface. Within the filaments are found membranous structures that are tubular or vesicular in appearance, called the vesicular chain [ $v c$, after (Tolbert and Morest 1982a)]. MACs are common elements within the calyx, and in many individual ultrathin sections are often located adjacent to synapses (Fig. 1D). Their apparent proximity to synapses and associations with membranous, often vesicular structures are suggestive of a role for MACs in synaptic function.

The adjacency of mitochondria-associated adherens complexes and synapses is revealed by serial section electron microscopy

To determine the spatial association between MACs and synapses, serial ultrathin sections (60-70 nm) of the MNTB, where calyces of Held are located, were collected and processed for electron microscopy. Representative serial sections from a small swelling are pictured in Figure $2 A-E$. In $A$, a MAC (M1) with its mitochondrion ( $m 1)$, mitochondrial plaque, vesicular chain $(v c)$, filaments, and punctum adherens is located at the left portion of the swelling. The vesicular chain of MAC-M1 $(A-C)$ is composed of vesicular and tubular membranes. A synapse $(B-E, s 1)$ lies adjacent to MAC-M1. The dense projections of $s 1$, thought to represent vesicle-docking sites, are evident in Figure 2C-E. Mitochondrion $\mathrm{m} 1$ forms a second MAC in the three sections after Figure $2 E$ (data not shown). The region of cell membrane containing MAC-M1 and s1 at the left part of the swelling is isolated by a region of wide separation between the presynaptic and postsynaptic membrane from another region, in the middle of the swelling, which contains another MAC (M2) and synapse (s2). These enlarged spaces have been noted in calyces of several species (rat,(Lenn and Reese 1966) bat, (Nakajima 1971; Petelina 1975); cat, (Jean-Baptiste and Morest 1975; Casey and Feldman 
1985)) and are called extended extracellular spaces (EESs) (Fig. 2, asterisks). MAC-M2 exhibits a more complex arrangement of vesicular and tubular membrane (Fig. $2 B-D$ ) that merges with a group of synaptic vesicles, some of which are associated with dense projections of s2 (Fig. $2 C-E$ ). The middle region of the swelling is separated by another EES from a third region of membrane apposition at the right of the swelling. This MAC (M3) also partly overlies its adjacent synapse (Fig. $2 C-E, s 3$ ). Therefore, the spatial relationship between MACs and synapses is complex and is best revealed by mapping the location of MACs and synapses on the presynaptic membrane surface.

Maps indicating the location of MACs and synapses were made in the swelling and stalk compartments of the calyx (Fig. $3 A, B$ ). Densities of the presynaptic and postsynaptic membrane, when viewed using magnification of at least $4000 \times$, could be classified as synapse or punctum adherens regions of MACs. Electron micrographs were scanned into a computer and aligned, and the membrane areas occupied by synapses and MACs were coded in dark gray and black, respectively. The membrane of the nerve terminal that did not face the postsynaptic cell was not included in the analysis, and the remaining membrane was unfolded into a flat sheet. The resulting en face maps revealed that MACs and synapses formed clusters that were separated by large distances. Inspection of the composite electron micrographs revealed that non-MAC or synapsecontaining membrane (coded in light gray) was primarily occupied by the EES. In the en face map of the swelling shown in Figure $3 A$, the clusters were composed of MACs forming a core surrounded by synapses, except at the periphery of the swelling where synapses were found without associated MACs. Inspection of the composite micrographs revealed that these were regions along the side of the swellings that faced somatic appendages of the MNTB cell. An intertwined arrangement of MACs and synapses was also found in stalks (Fig. 3B). Here, MACs were interspersed with synapses and found in regions that apposed somatic appendages (Fig. 3B, right side). 
The adjacency of MACs and synapses was quantified by measuring the distance, on the en face diagram, from the vesicular chain or mitochondrial plaque portion of the MAC (Fig. $3 A, B$, small white circles) to the nearest synapse. The mean distance from these components of the MAC to the edge of the nearest synapse was $202 \pm 94.9 \mathrm{~nm}$, or approximately five synaptic vesicle diameters (Fig. 3C). However, the distance from some synapses to the nearest MAC could approach $1 \mu \mathrm{m}$ (Fig. 3D), consistent with the absence of MACs in some regions of membrane apposition (Fig. 3A). These distances were measured from five series of sections taken from four calyces in two animals, each having a composite thickness of 1.3-4.8 $\mu \mathrm{m}$. The same spatial relationship between MACs and synapses was also evident in another five reconstructions, taken from five calyces (four of which are from a third animal), each having a composite thickness of at least $0.5 \mu \mathrm{m}$.

Construction of en face diagrams from serial sections affords the opportunity to estimate the number of synapses and MACs in each calyx of Held. In each en face diagram, individual synapses and puncta adherentia were outlined, and their surface area was calculated by summing the lengths measured from the micrographs and then multiplying by the section thickness. The average sizes for each en face diagram are reported in Table 1. Overall, synaptic junction surface area averaged $70,079 \mathrm{~nm}^{2}$ and occupied $11.6 \%$ of the presynaptic membrane surface, whereas MACs surface area averaged $54,622 \mathrm{~nm}^{2}$ and occupied $5.8 \%$ of the presynaptic membrane surface. The postsynaptic MNTB cell body is nearly spherical in shape and $\sim 35 \mu \mathrm{m}$ in diameter. Using published measurements that the calyx covers on average $38 \%$ of the postsynaptic cell body in cats [our calculation from (Smith et al. 1991; Smith et al. 1998)], we estimate that the calyx of Held contains 2400 synaptic junctions and 1600 MACs. The number of synapses is larger than that estimated for large synaptic end-bulb terminals in the ventral cochlear nucleus (Ryugo et al. 1996). 


\section{Vesicular and tubular membrane structures in the nerve terminal}

Arrangements of membrane within the MAC can take the form of two to four linked vesicular structures or, more commonly, of combinations of tubular and vesicular membrane that can extend along the sides of the mitochondrion away from the cell membrane (Fig. 4F, dotted arrow). The tubular networks are curved and branched, have a twisted appearance, and appear to be fused or in the process of budding round vesicles that are the size of synaptic vesicles (Fig. $4 B, E, F$, arrowheads). Some membranous structures are non-round, with profiles that are approximately the size of synaptic vesicles (vesicular chain of M1 in Figs. $2 A$ and $4 E)$.

Coated vesicles in the nerve terminal, presumably recaptured from the presynaptic membrane, are the same size as synaptic vesicles and can be found in proximity to and fused to tubular networks associated with MACs (Figs. 2E, $4 A, C$, solid arrows). Coated vesicles appear to emerge from synaptic zones or adjacent areas of presynaptic membrane (Fig. 4A,F, open arrows). Synaptic zones also contain structures that could represent vesicle fusion sites or the early stages of vesicle reuptake (Fig. $2 E$, open arrows at $s 2$ ). The EES appears to be a common site of membrane recycling (Figs. $2 C, 4 D$, open arrows), as originally observed using scanning (freeze-fracture) and transmission electron microscopy (Heuser and Reese 1973; Gulley et al. 1978). All areas of the presynaptic nerve terminal membrane seem capable of endocytotic events, so we measured the prevalence of coated pits, presumably representing endocytosing vesicles, in different regions of the presynaptic membrane. Most coated pits were found immediately adjacent to the synapse $(27 \%)$ or at the edge of the EES $(42 \%)$. Large endocytotic coated pits and vesicles are often located post-synaptic to the EES or associated with membrane that faces away from the MNTB cell and are probably not involved in synaptic vesicle recycling (Fig. $2 C$, solid arrows). On the basis of the presence of MAC and synapse-containing regions of membrane apposition, flanked by EES and endocytotic profiles, we propose that the calyx is composed of numerous activity modules, each with a self-contained system of vesicle recycling. 


\section{Are there subpopulations of mitochondria in the calyx of Held?}

Mitochondria that form MACs have structural specializations in the precise orientation of their cristae and flattened edge where they abut the mitochondrial plaque. The branched and curving appearance of mitochondria in individual electron micrographs (Fig. 2, m3) was suggestive that MAC-forming mitochondria were more complex structurally than other mitochondria in the nerve terminal. To investigate this hypothesis, mitochondria were reconstructed across serial sections, and their complexity was determined by counting the number of tips, or ends, of processes. For example, the electron micrograph in Figure $5 A$ reveals what appear to be four presynaptic mitochondria. In the next two sections in the series (Fig. 5B,C), three of the profiles (labeled $m$ in Fig. $5 A$ ) merge into a single mitochondrion. Three-dimensional reconstruction of this mitochondrion (Fig. $5 D, E)$ reveals the three limbs seen in the serial sections (labeled $m$ ). The mitochondrion has four tips and forms two MACs (punctum adherens, green; mitochondrial plaque, orange) that are adjacent to synapses (red). The analysis of geometric complexity revealed distinct populations of mitochondria in the calyx. Most mitochondria did not form MACs and had two ends and were therefore vermiform in shape, although they may have a bend or curve along their length (Table 2). Mitochondria that formed MACs had a greater tendency to be complex $(p<0.001$; Fisher's Exact Test), could form MACs along more than one process, and often had a process that extended away from the membrane into the core of the stalk or swelling (Fig. 2, m3; Fig. 5F,G, top limb of mitochondrion). Location of a mitochondrion within $200 \mathrm{~nm}$ of the presynaptic membrane, in a region of presynaptic and postsynaptic membrane apposition, was a strong predictor that it formed a MAC.

\section{Structural correlates of vesicle release probability}

The multitude of synapses in a single calyx and the requirement for the calyx to transmit high rates of activity to the MNTB cell may dictate that not all synapses release a vesicle with each invading action potential. MAC-associated synapses may have greater capacity to sustain vesicle release during the later stages of a 
spike train. The number of synaptic vesicles that are in position to be released is thought to be the structural equivalent of the readily releasable pool (von Gersdorff et al. 1996) (Schikorski and Stevens 1997). We counted in serial sections the number of synaptic vesicles that were docked in calyceal synapses to determine whether MAC-associated synapses have a greater number of docked vesicles. Synaptic vesicles were classified as docked if there was no resolvable distance between their membrane and the presynaptic membrane or dense projections of the synaptic junction (Fig. 6A). There was no difference in the mean number of docked synaptic vesicles in MAC-associated synapses $(2.6 \pm 1.71)$ from those synaptic junctions positioned farther $(>500 \mathrm{~nm})$ from the MAC (2.6 \pm 1.5$)$, and therefore their distributions are combined (Fig. 6B). The low-calcium transcardial rinse that we used may have stopped vesicle fusion before fixation, but perhaps permitted reloading of vesicle docking sites in both MAC-associated and MAC-independent synapses. Using the estimate that each action potential evokes release of 0.2 of the readily releasable synaptic vesicle pool (Schneggenburger et al. 1999), we suggest that synapses having three or more docked vesicles are most likely to release a quantum of neurotransmitter upon depolarization (Fig. 6B, rightmost portion of distribution). Multiplying our distribution of the docked vesicles per synapse by our estimate of the number of synaptic junctions in the calyx, we calculate that the calyx contains 6200 readily releasable synaptic vesicles. Although the number of docked vesicles per synapse is a factor of 5-7 lower in the calyx than in hippocampal boutons (Harris and Sultan 1995; Schikorski and Stevens 1997), the great majority of hippocampal boutons contain only one active site. The large number of synapses in the calyx terminal may provide enough of a safety net that limits the rate at which individual synapses need to refill their docking sites, especially if the released fraction of vesicles declines during a spike train. Investigation of the effects of stimulus history on vesicle priming will require development of an in vitro preparation with appropriate ultrastructure preservation that permits counting of docked vesicles. 


\section{Discussion}

Our fundamental finding is that the MAC is intertwined with synapses and is found in the prototypical fast, glutamatergic nerve terminal, the calyx of Held. Because of its proximity to synapses and the component organelles from which it is constructed, we hypothesize that the MAC plays a central role in high rate, temporally precise neurotransmission that is a hallmark of the calyx terminal (Guinan, Jr. and Li 1990). The presence of coated, presumably endocytosing, vesicles adjacent to fusion sites and in locations encircling MAC/synapse clusters suggests that the calyx is composed of multiple activity zones, each being an engine for releasing and recharging its vesicle store. Increasingly, evidence points to a central role for mitochondria in regulating exocytosis on a fast time scale (Kaftan et al. 2000) (see below). The MAC is the most highly ordered structural arrangement of which we are aware, linking mitochondria to synaptic junctions, and it may play an integral role in synaptic transmission in the calyx.

\section{Auditory brainstem circuits}

The presence of MACs in end-bulbs and modified end-bulbs of Held in the cochlear nucleus (Cant and Morest 1979; Tolbert and Morest 1982a), in large collateral terminals of calyceal axons in the superior olive (Spirou et al. 1998), and in calyces of Held implies their importance in neural circuits that subserve the early stages of sound localization (Fig. 7A). MAC-containing end-bulbs activate spherical bushy cells (Cant and Morest 1979), which excite binaural cells of the medial superior olive (MSO) bilaterally and lateral superior olive ipsilaterally. MAC-containing modified end-bulbs activate globular bushy cells (Tolbert and Morest 1982a), which in turn excite ipsilateral cells of the lateral nucleus of the trapezoid body (LNTB) via MAC-containing modified end-bulbs (Spirou et al. 1998 ) and contralateral cells of the MNTB via calyces (now shown to also contain MACs). LNTB cells provide inhibitory input to the MSO (our unpublished data), and MNTB cells inhibit cells of the MSO and LSO (Moore and Caspary 1983; Smith 1995). Therefore, MAC-containing terminals drive both excitatory and inhibitory inputs to binaural comparator neurons. 
MACs are also present in rat calyces of Held (our unpublished data) [also see (Casey and Feldman 1985), their Figs. 5, 8, 19, 20, 22, although the presence of MACs was not noted by these authors], but their prominence and association with synapses has not been determined. MACs have been described in terminals of some olivo-cochlear fibers that project from cells of the superior olive onto cell bodies of spiral ganglion neurons [monkeys: (Kimura et al. 1987); humans: (Rask-Andersen et al. 2000)]. It is not known whether these olivo-cochlear neurons generate high rates of temporally synchronous activity, in common with other MAC-containing auditory system neurons. The only other report of MACs of which we are aware is by Gray (Gray 1963) in his original description of this structure in the spinal cord. The cell type giving rise to nerve terminals containing MACs was not determined, nor have we found subsequent descriptions of this structure in more recent studies of the spinal cord. Structures bearing similarity to MACs, called filamentous contacts, have been described in thalamic relay nuclei (Colonnier and Guillery 1964; Lieberman and Spacek 1997). However, filamentous contacts are segregated from most synapses in the terminal. They do exhibit a vesicular chain-like membrane (called agranular reticulum) located between mitochondria and the presynaptic punctum adherens. The mitochondrial cristae are less strictly oriented than in the MAC and occasionally filaments extend from the reticular membrane to the punctum adherens, but there is no mitochondrial plaque. Filamentous contacts feature a similar network of punctum adherens, filaments, and reticulum in the postsynaptic cell, incorporate tight junctions, and are also found at dendrodendritic and somasomatic junctions. They most likely play roles in intercellular communication that are distinct from MACs.

\section{Vesicle recycling and the readily releasable pool}

The vesicular chain component of MACs and synapses points to roles in vesicle membrane recycling. Generally two modes of vesicle recycling are thought to occur at nerve terminals: full fusion of vesicles with the presynaptic membrane followed by clathrin-mediated endocytosis (Heuser and Reese 1973) and partial 
fusion of vesicle membrane followed by rapid, clathrin-independent disassociation from the presynaptic membrane (also known as "kiss-and-run") (Ceccarelli et al. 1973); for review, see (von Gersdorff and Matthews 1999). Clathrin-coated endocytotic vesicles may fuse with endosomal intermediates that bud new synaptic vesicles, but recent evidence indicates that fusion with endosomes may not be part of the life cycle of the vesicle (Murthy and Stevens 1998), even in the case of clathrin-coated vesicles (Takei et al. 1996). Coated vesicles are a common feature in calyx terminals and bud from synaptic zones, but more frequently from edges of the EES that form a border around MACsynapse clusters. Coated vesicles are frequently located near and sometimes appear fused with the vesicular chain component of the MAC, so recycling through endosomal intermediates may support replenishment of vesicle stores in the calyx of Held. Therefore in the calyx the distinction between recycling and reserve (to sustain high activity rates) vesicle pools may be blurred (Kuromi and Kidokoro 1999). Kiss-and-run may also be at play in calyx terminals but cannot be resolved with certainty using electron microscopy.

The calyx, like many other central synapses, exhibits frequency-dependent synaptic depression that is caused mainly by depletion of the readily releasable pool of synaptic vesicles (Borst et al. 1995; von Gersdorff and Matthews 1997) (Wang and Kaczmarek 1998). In rats, the readily releasable pool size in the calyx was estimated to be 700 vesicles (Schneggenburger et al. 1999). Using docked vesicles as a morphological indicator, we estimate that 6200 vesicles constitute the readily releasable pool in calyces, which may reflect the larger size of calyces in cats than in rats.

\section{Hypothesized functions of the MAC}

The conglomerate elements of the MAC result in a structure that, because of its uniqueness, is somewhat enigmatic in nature. In this section we offer several hypotheses (schematized in Fig. 7B) about the function of several MAC components and characteristics as a framework for investigating MAC functions in large auditory system nerve terminals. 


\section{Vesicular chain}

Recovery from synaptic depression of postsynaptic current amplitude is more rapid at high rates of stimulation at calyceal and hippocampal synapses (Stevens and Wesseling 1998; Wang and Kaczmarek 1998). Despite the reduced amplitude of postsynaptic currents, postsynaptic action potentials are reliably generated in brain slices at stimulus rates exceeding $500 \mathrm{~Hz}$ (Wu and Kelly 1993). We hypothesize (Fig. 7B, \#1) that vesicular chain membrane provides a reservoir for rapid formation of new vesicles under conditions of sustained, high rates of activity.

\section{Mitochondrion, mitochondrial plaque, and filaments: a system to regulate calcium levels}

In the calyx, multiple calcium channels open to induce the release of single quanta, so the calcium load on the terminal can be very high (Borst and Sakmann 1999a). Accumulating evidence indicates that mitochondria in nerve terminals, especially those nearest the synapse, increase their calcium conductance and sequester calcium during a train of impulses (David et al. 1998; Jonas et al. 1999; Pivovarova et al. 1999; Zenisek and Matthews 2000). Activity-dependent changes in presynaptic calcium concentration can reach 5-10 $\mu \mathrm{M}$ at $200 \mathrm{~nm}$ from the synapse (Neher 1998) and are likely detected by the mitochondrial component of the MAC, tethered at about that distance. Indeed, immediately after tetanic pulse trains, recovery from depression in the calyx is independent of membrane calcium currents (Forsythe et al. 1998) and may be caused in part by calcium re-release from MAC components. We hypothesize (Fig. 7B, \#2) that the mitochondrial (and perhaps vesicular chain) components of the MAC are positioned precisely by the mitochondrial plaque and filaments to cooperate with other calcium-buffering entities in the terminal. Proper calcium buffering will affect facilitation or depression of vesicle release during successive action potentials (Forsythe et al. 1998; Bellingham and Walmsley 1999; Borst and Sakmann 1999b; Wu and Borst 1999). 


\section{Mitochondrial geometry and cristae: an intracellular conduit for calcium movement}

Mitochondria most likely participate with endosomal structures, such as endoplasmic reticulum, in an elaborate calcium-buffering network that can shuttle calcium signals through the cytoplasm within organelles (Babcock et al. 1997) (Rizzuto et al. 1998). We hypothesize (Fig. 7B, \#3) that the complex geometry of many MAC-associated mitochondria, perhaps together with the vesicular chain, may subserve this function, shuttling calcium from one activity module across the EES to another activity module and thereby affecting release probability at synapses several micrometers away. Furthermore, we propose (Fig. 7B, \#4) that the cristae of MAC-associated mitochondria are oriented perpendicular to the membrane to facilitate diffusion away from the synaptic region to other ends of the mitochondrion. Cristae of neuronal mitochondria can form lamellar, rather than tubular, structures (Perkins et al. 1997) that should have high capacity for calcium diffusion.

\section{Mitochondrial biochemistry: possible source of energy and neurotransmitter}

Tethering mitochondria near synaptic structures may link the upregulation of Krebs' cycle enzymatic activity by calcium to increased ATP production (Denton and McCormack 1980) to support synaptic mechanisms activated by neural activity. Also, mitochondria can manufacture glutamate from $\alpha$-ketoglutarate, which in pancreas has a direct effect on insulin secretion (Maechler and Wollheim 1999). We hypothesize (Fig. 7B, \#5) that MAC-associated mitochondria may manufacture glutamate for use as a neurotransmitter, which is then used to refill recycled synaptic vesicles (Maycox et al. 1988). 


\section{Punctum adherens: synaptic stabilization and development}

We have hypothesized previously (Fig. $7 B, \# 6$ ) that the punctum adherens component of the MAC provides needed stability to the calyx-MNTB neuron contact to sustain high rates of neural activity and high-capacity membrane recycling (Spirou et al. 1998). The punctum adherens component of the MAC may play an additional role during early formation of the synapse. The cadherin protein family may be sufficiently diverse (Kohmura et al. 1998; Brose 1999; Serafini 1999) to underlie the tonotopic pattern by which calyceal growth cones innervate the appropriate MNTB cells to preserve this fundamental organizing principle of auditory neural circuits (Fig. $7 B, \# 7$ ). A similar role in forming retinotopic connections was proposed for filamentous contacts (Colonnier and Guillery 1964). The development of suitable experimental preparations and probes will form the basis for investigating these and other hypotheses about functions of this atypical organelle assembly. 
Acknowledgements

We thank Albert Berrebi for expertise in PEP-19 immunocytochemistry, Brian Pope and Marcia Feinberg for technical assistance, and Erika Hartweig for training in the collection of serial sections. We thank members of the Sensory Neuroscience Research Center, William Wonderlin, and Philip Smith for critical review of this manuscript, Peter Mathers for insightful discussions of data, and George Augustine for reading an earlier version of this paper. 


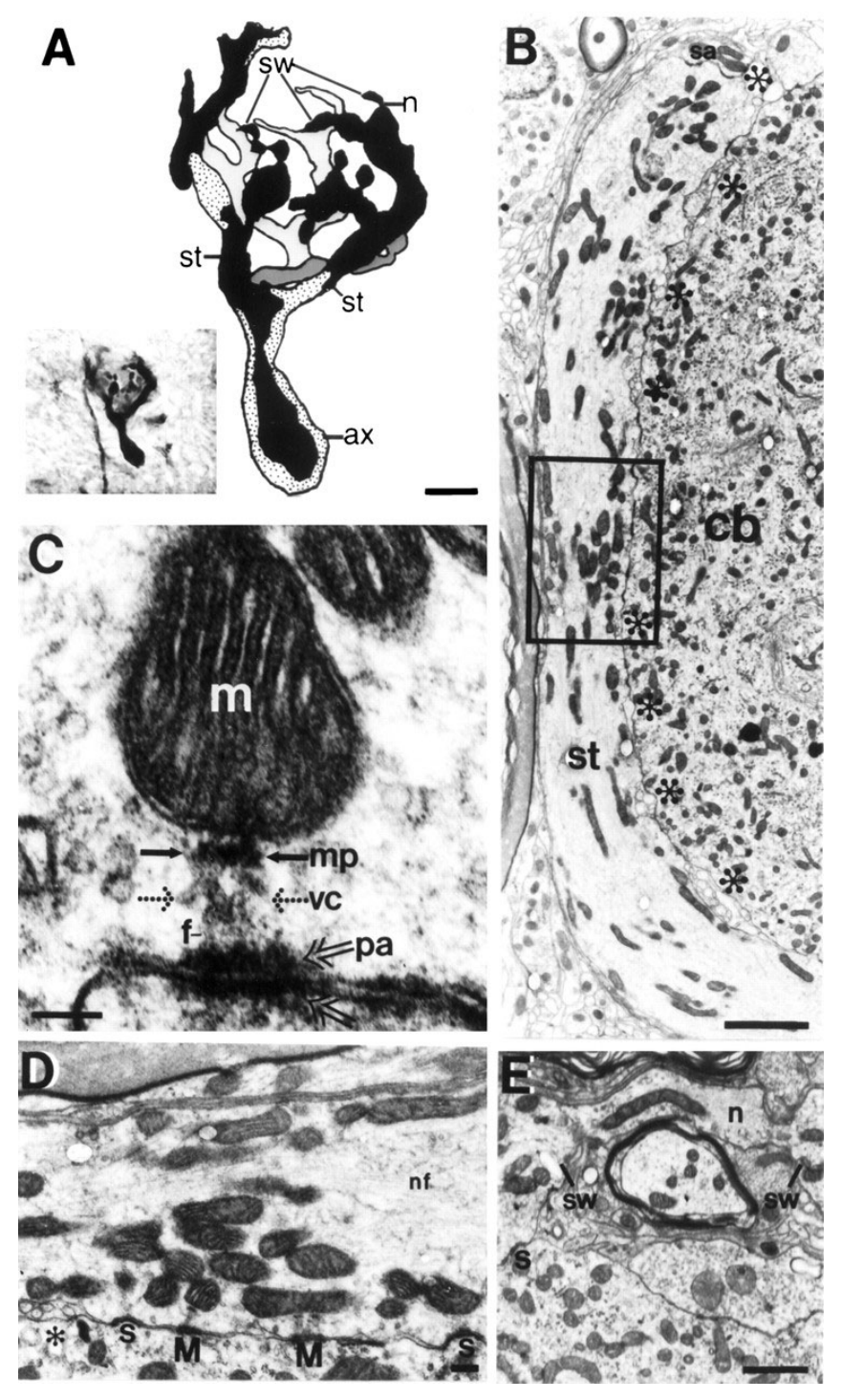

Figure 1. The calyx of Held is a large, complex nerve terminal that envelops the postsynaptic neuron and has multiple synaptic sites.

A, Camera lucida and light micrograph (inset) of a calyx revealed by antibodies against the putative calcium binding protein PEP-19. The calyceal axon (ax) branches into four stalks (st, two of which are labeled), which themselves branch through narrow necks (n) of varying length into bulb-shaped swellings (sw) of various sizes. Black and stippled areas represent more superficial focal planes than light and dark gray areas. Scale bar, $5 \mu \mathrm{m}$. B, Electron micrograph of a stalk (st) portion of the calyx of Held that wraps halfway around the MNTB cell body 
(cb). Regions of close apposition between presynaptic and postsynaptic membrane alternate with regions of wide separation, called the EES (next to asterisks). The end of the stalk contacts a somatic appendage (sa). Boxed region is shown in D. Scale bar, $2 \mu \mathrm{m}$. C, Elements of the MAC: mitochondrion (m), mitochondrial plaque ( $\mathrm{mp}$, solid arrows), filaments (f), punctum adherens (pa, open arrows), and vesicular chain (vc, dotted arrows). Scale bar, $100 \mathrm{~nm}$. D, Electron micrograph of the boxed outline in $B$ reveals mitochondria surrounding a central core of neurofilaments (nf). Synapses (s) and MACs (M) are interspersed along the stalk and occupy most of the regions of apposition between presynaptic and postsynaptic membranes. Asterisk underlies the extended extracellular space. Scale bar, $250 \mathrm{~nm}$. E, Electron micrograph of a neck (n) that connects two swellings (sw), the latter of which contains synapses (s). Scale bar, $1 \mu \mathrm{m}$. 


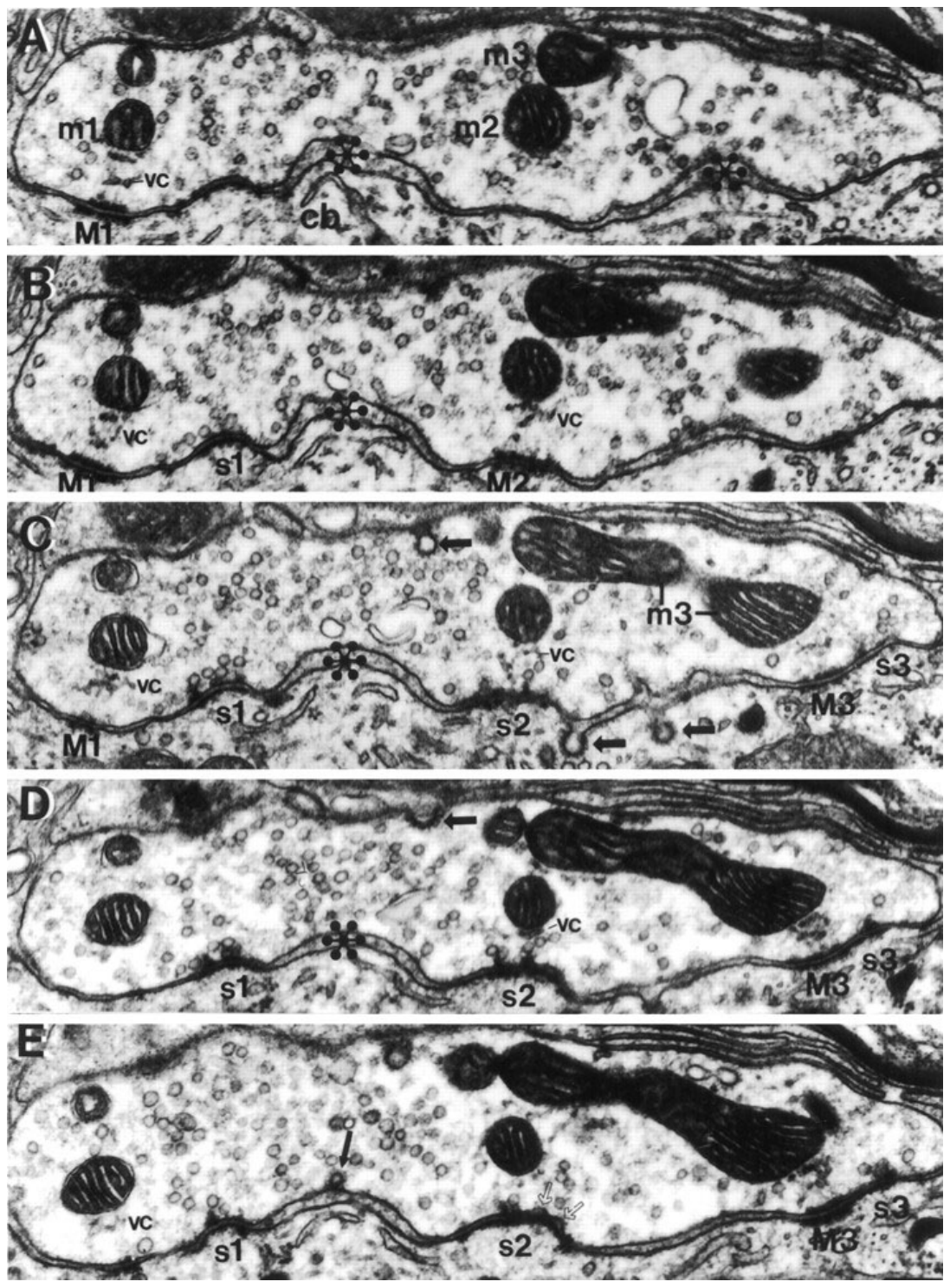

Figure 2. Serial sections indicate that MACs are adjacent to synapses.

These serial sections of a swelling in contact with an MNTB cell body $(c b)$ reveal three regions of membrane apposition (left, middle, and right) separated by an EES (asterisks; rightmost EES is only marked in $A)$. Three MACs $(M 1, A-C ; M 2$, $B ; M 3, C-E)$ and their associated mitochondria ( $m 1, m 2$, and $m 3$ in $A$; $m 3$ labeled again in $C$ ) and vesicular chain ( $v c)$ are adjacent to synapses (s1, s2, and $s 3$ ). Large coated vesicles are budding from the side of the terminal facing away from the MNTB cell and postsynaptically from the extended extracellular space $(C, D$, solid arrows). Bumps in the presynaptic membrane ( $E$, open arrows) at the 
synapse could indicate vesicle fusion or retrieval sites. A coated vesicle ( $E$, solid arrow) is located adjacent to the presynaptic membrane. Scale bar (shown in $E$ for $A-E): 200 \mathrm{~nm}$. 


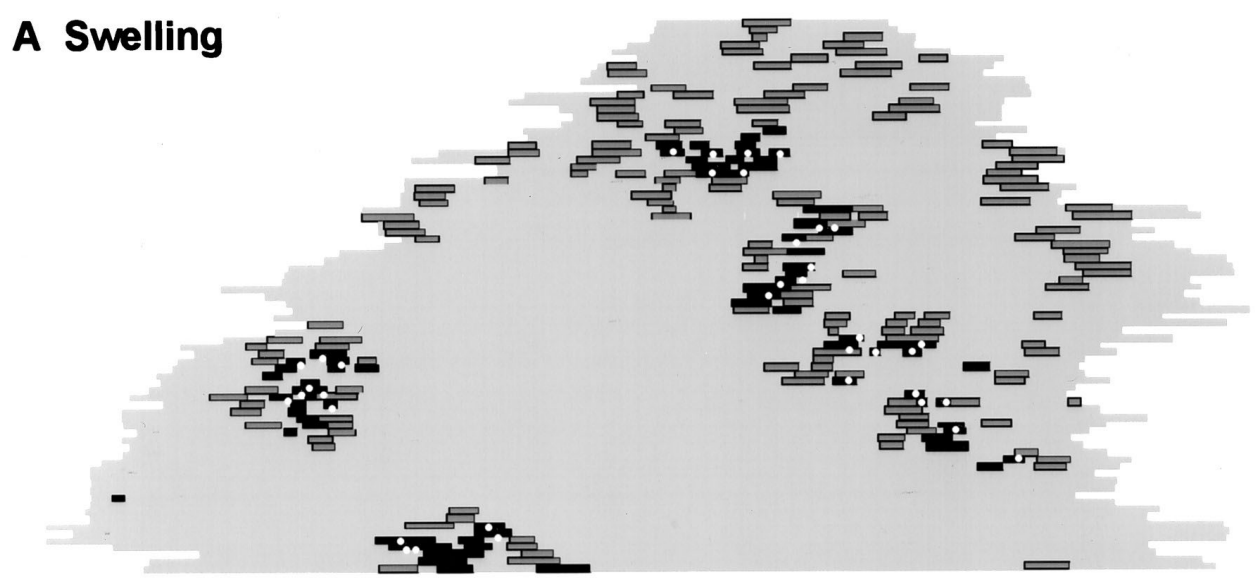

B Stalk

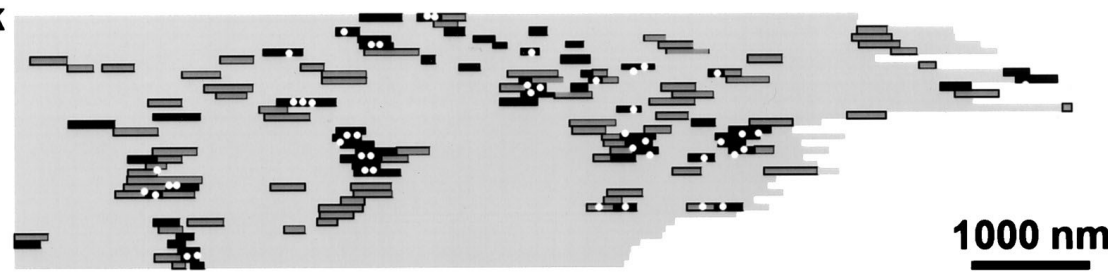

C
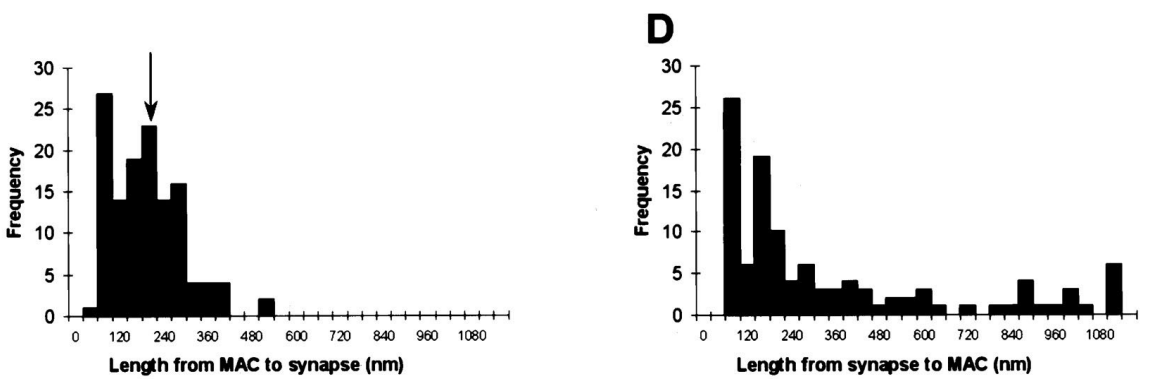

Figure 3. The interdigitated spatial relationship of synapses and MACs is revealed in en face views of the flattened presynaptic membrane.

Lengths of membrane that contain synapses are coded dark gray, and punctum adherens components of MACs are coded black. Other regions of membrane, primarily composed of extended extracellular space, are coded light gray. Small white circles represent the vesicular chain and mitochondrial plaque components of MACs. A, En face diagram through one swelling portion of a calyx (77 serial sections). B, En face diagram through one stalk portion of a calyx (36 serial sections). C, The shortest distance from the mitochondrial plaque or vesicular chain components of MACs to synapses averaged $202 \pm 94.9 \mathrm{~nm}$ (arrow). D, The shortest distance from synapses to the nearest MAC often could exceed $500 \mathrm{~nm}$, 
reflecting a category of synapses that are not located near MACs (as shown in A). 

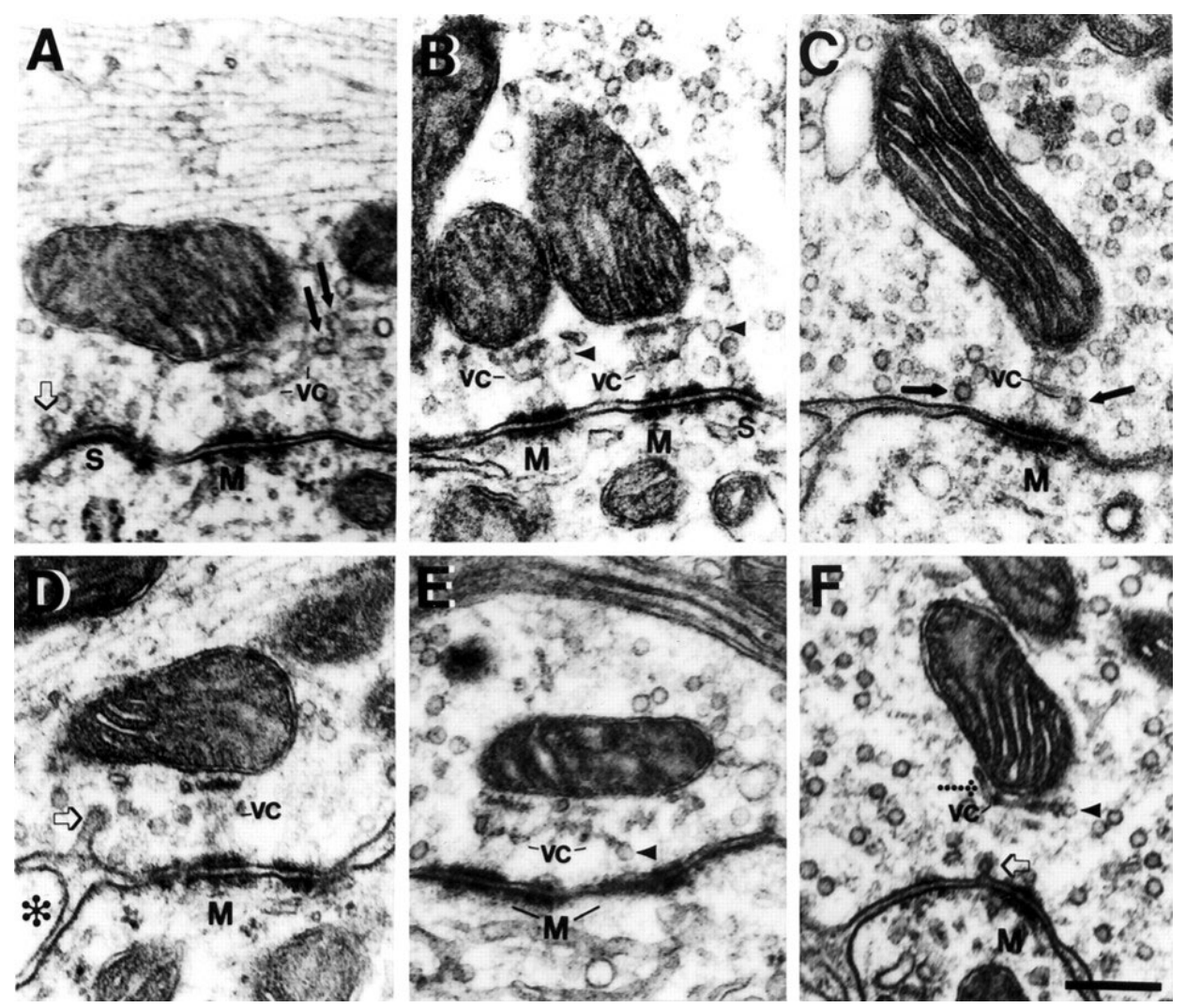

Figure 4. Coated and noncoated vesicles appear fused with the vesicular chain component of the MAC.

$A$, A mitochondrion forms a MAC $(M)$ adjacent to a synapse $(s)$. The vesicular chain $(v c)$ leads into a branched tubular network adjacent to the mitochondrion, to which coated vesicles (solid arrows) appear fused. A coated vesicle at the 7synapse (open arrow) appears to be in the process of endocytosis. $B$, Two mitochondria form side-by-side MACs $(M)$; the edge of a synapse $(s)$ is adjacent to one of the MACs. The vesicular chain forms a tubular or flattened structure to which vesicles appear fused (arrowheads). C, Coated vesicles (solid arrows) are located next to the vesicular chain and just inside the presynaptic membrane. $D$, A coated pit (open arrow) is located at the end of a membrane invagination, at the edge of the EES (asterisk is within glial process inside the EES), and near a MAC. E, F, Noncoated vesicles (arrowheads) appear fused to the vesicular chain, which appears flattened in $E$ and tubular, bending along the side of the 
mitochondrion (dotted arrow), in F. A coated vesicle (open arrow) appears to be in the final stage of budding from the presynaptic membrane. Scale bar (shown in $F$ for $A-F): 250 \mathrm{~nm}$. 

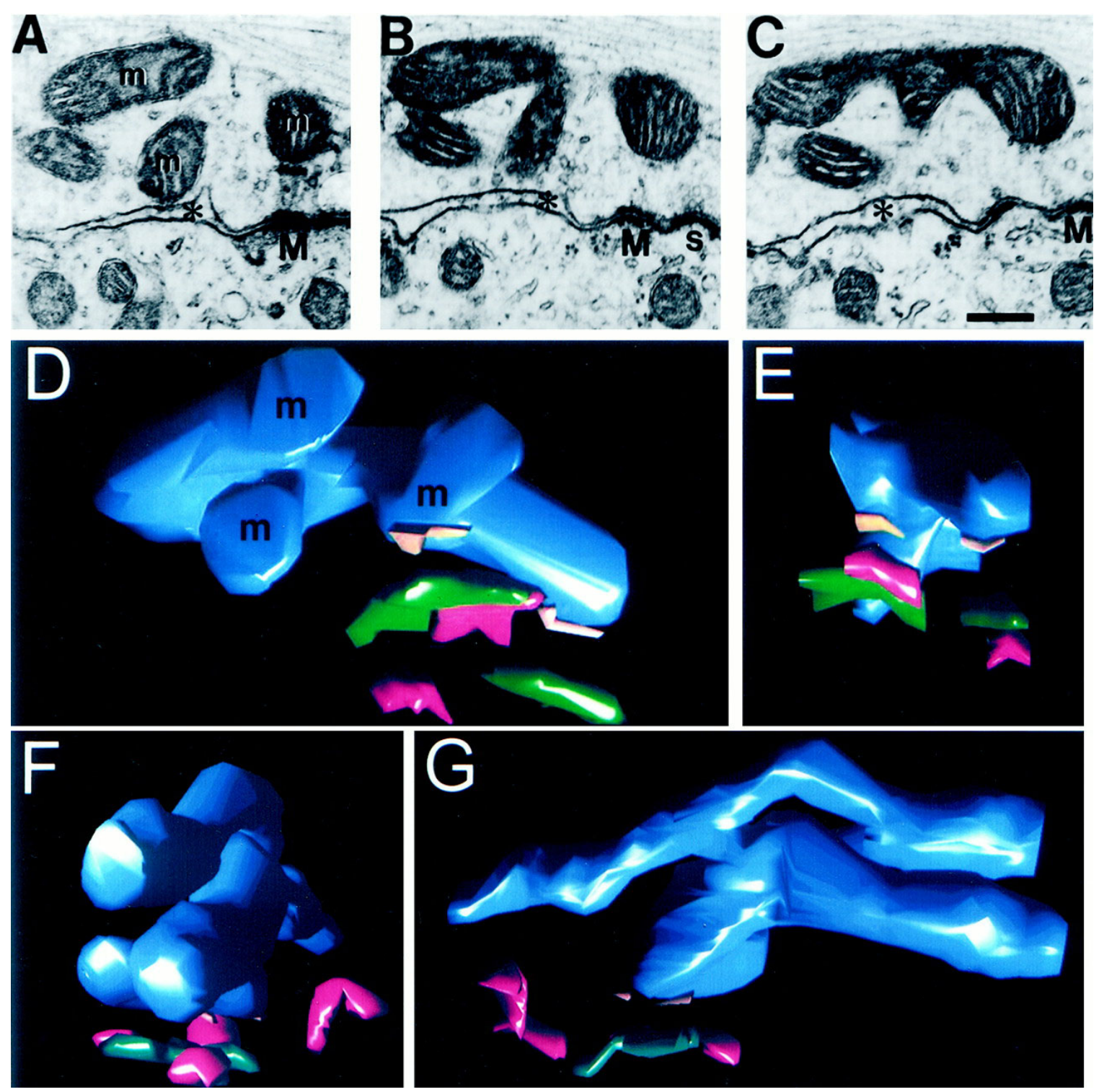

Figure 5. Mitochondria can form more than one MAC and have complex geometry.

$A-C$, Three limbs of a single, MAC-forming mitochondrion $(m)$. One $\operatorname{MAC}(M)$ and its adjacent synapse (s) are pictured. Asterisks indicate EES. Scale bar, $250 \mathrm{~nm}$. $D, E$, Orthogonal views of a three-dimensional reconstruction of the complex 
mitochondrion pictured in $A-C$. The mitochondrion (b/ue), mitochondrial plaques (orange bars), puncta adherentia (green), and synapses (red) are depicted. F, G, Three-dimensional reconstruction of a second complex mitochondrion (end and side views) with its associated MAC and synaptic structures. The two long limbs of this mitochondrion extend $>1 \mu \mathrm{m}$ in length. 

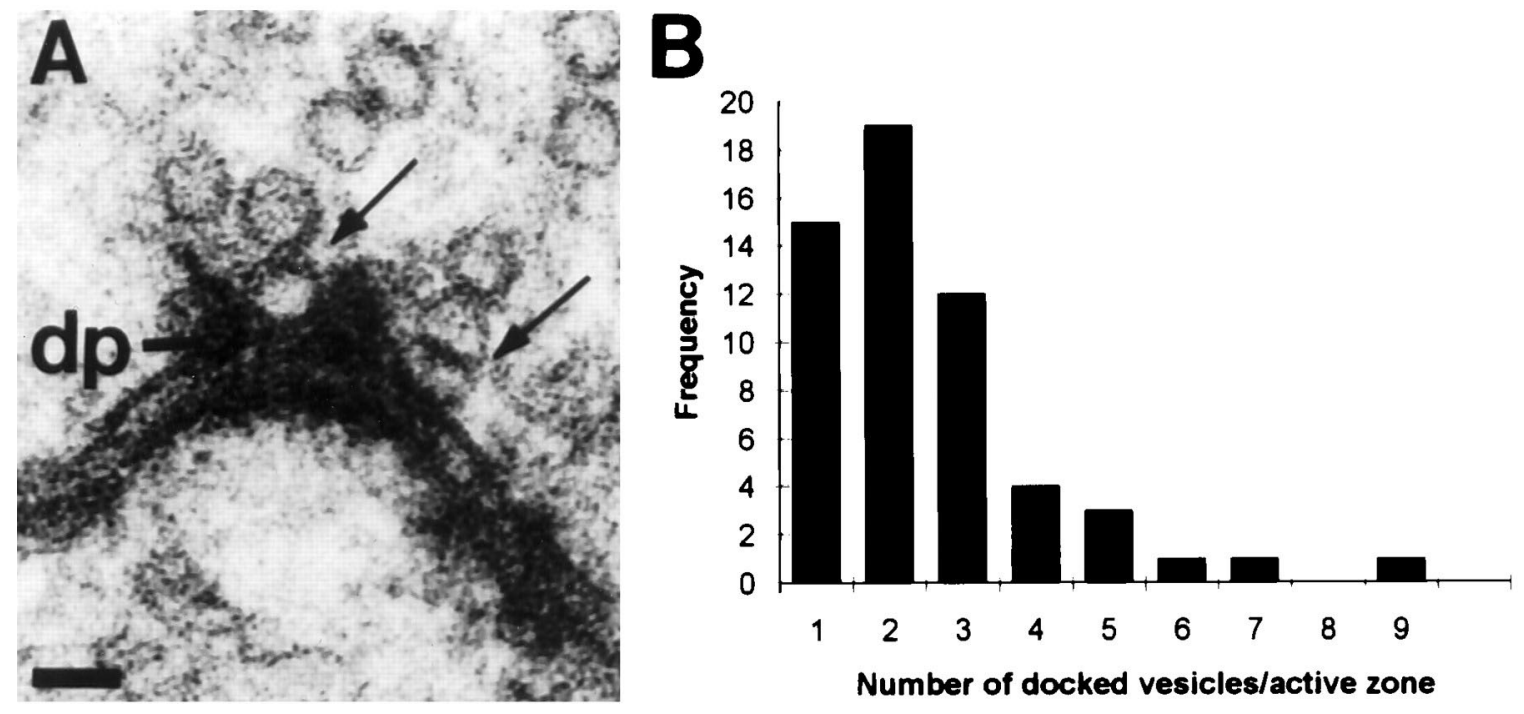

Figure 6. Synapses vary in the number of docked vesicles.

$A$, Two synaptic vesicles (arrows) are docked at the presynaptic membrane, alongside hypothesized docking structures called dense projections $(d p)$. $B$, Distribution of the number of docked vesicles found in individual synapses. Mean number of docked vesicles is $2.53 \pm 1.61 ; n=56$. 


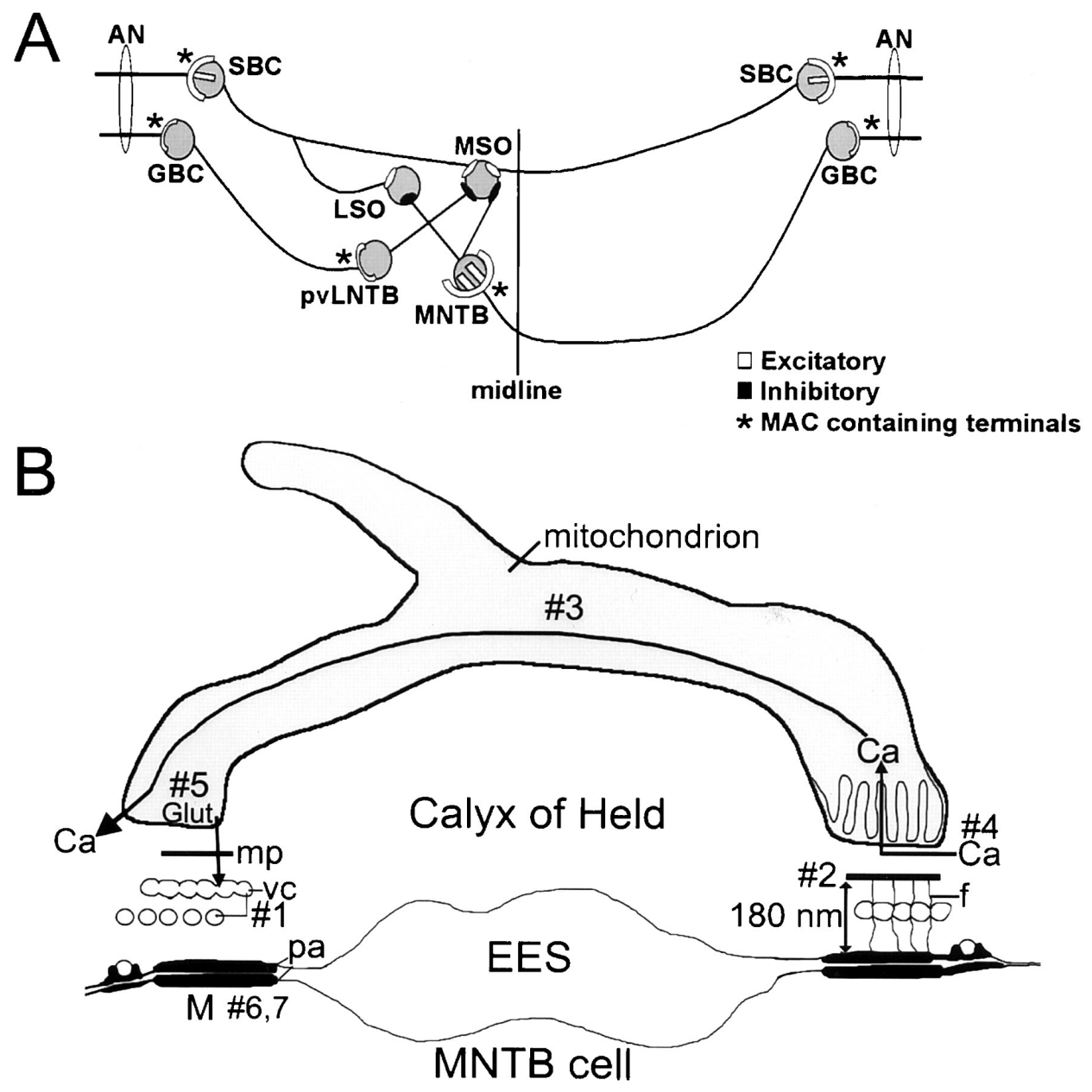

Figure 7. Auditory brainstem circuitry.

$A$, Auditory nerve $(A N)$ fibers end in large, MAC-containing nerve terminals $\left({ }^{*}\right)$ onto spherical $(S B C)$ and globular $(G B C)$ bushy cells of the cochlear nucleus. GBCs project ipsilaterally, via MAC-containing modified end-bulbs ( $\left.{ }^{*}\right)$, to cells of the posteroventral lateral nucleus of the trapezoid body ( $p V L N T B)$ and contralaterally, via MAC-containing calyces $\left({ }^{*}\right)$, to cells of the medial nucleus of the trapezoid body (MNTB). Cells of the medial superior olive (MSO) and lateral superior olive (LSO) compare bilateral excitatory (from spherical bushy cells) and inhibitory (from LNTB and MNTB cells) inputs to assign a location to a sound source in space. $B$, Hypothesized functions of the MAC $[M$; composed of a mitochondrial plaque $(m p)$, vesicular chain $(v c)$, filaments $(f)$, punctum adherens (pa), and a mitochondrion]. MNTB postsynaptic cell is at bottom; EES, extended extracellular space. Hypotheses: \#1, The vesicular chain is a reservoir for newly 
formed synaptic vesicles. \#2, The close proximity of mitochondria to the presynaptic membrane allows for calcium buffering at synapses. \#3, Calcium $\mathrm{Ca}$ ) is shuttled through the mitochondrion to other active zones of the terminal. \#4, Orientation of cristae facilitates calcium diffusion. \#5, Mitochondria support neurosecretion by manufacture of glutamate (G/ut) neurotransmitter and refilling synaptic vesicles. \#6, Stabilization of the presynaptic membrane during high rates of activity is achieved by the punctum adherens. \#7, Puncta adherentia determine tonotopic innervation of the MNTB during development. 
Chapter 3: Maturation of membrane electrical properties of neurons in the medial superior olive of the gerbil. 


\section{Abstract}

The maturation of the electrical membrane properties of medial superior olive (MSO) neurons has not been well studied. We performed current clamp recordings from neurons of the principal cell column of the MSO in postnatal day (PD) 4-19 gerbils (Meriones unguiculatus) to examine the postnatal changes in the electrical membrane properties and inhibitory postsynaptic potentials (IPSPs). MSO neurons were placed into two groups: postnatal day (PD) 4-11 and PD 12-19 based upon the onset of hearing in the gerbil (PD 12). The MSO cell column was found to contain two different cell types, those that respond phasically or tonically to rectangular pulses of depolarizing current. The proportion of phasic cells dramatically increased after the first week of life and between PDs 4 and 19, the input resistance (Ri) of phasic cells (mean Ri= 205 $\mathrm{M} \Omega$ in PD 4-11 and $56 \mathrm{M} \Omega$ in PD 12-19) and membrane time constant of phasic cells (time constant $=16.8 \pm 4.7$ in PD $4-11$ and $2.3 \pm 0.9 \mathrm{~ms}$ in PD 12-19) decreased with age.

The developmental properties of inhibitory neurotransmission in the MSO has not been well-studied. We found that evoked IPSPs became faster with increasing age (IPSP time constant $=29.6 \pm 7$ and $3.0 \pm 2.0 \mathrm{~ms}$, PD 4-11 and 1219 , respectively). There was a strong correlation between the decrease in the membrane time constant and the IPSP recovery time constant (correlation coefficient, $R^{2}=.88$ ) indicating that the increased speed of IPSPs between PDs 4-19 is mainly due to the membrane time constant becoming faster. 


\section{Introduction}

Our investigation of cellular events that underlie encoding of sound location relies on techniques that are most easily performed on young animals. Therefore, as a first step we investigated the maturation of MSO neurons between postnatal day (PD) 4-19, to identify the youngest age range at which cellular properties become adult-like and on which to focus our studies.

Several studies of the MSO have employed intracellular recordings of adult neurons in brain slices by using sharp electrodes that penetrate the cell column to record from neurons that are not in view (Smith 1995). The narrow cell column of the MSO renders this approach to be of low yield in achieving stable recordings. In addition, intracellular recordings with sharp electrodes are more susceptible to movement artifacts that result from positioning other probes near the recording site. Visualization of cells in the slice with differential interference contrast (DIC) optics under infrared illumination is one means to increase the yield from this type of experiment. However, cells are most clearly identified in young animals because axons are usually unmyelinated and therefore do not scatter light, as happens in adults. Therefore, our strategy was to identify the age after which electrical properties of the cell were mature so that interpretation of data was not confounded by consideration of developmental processes. Since our investigation of ITD coding by MSO cells was focused on the roles of potassium channels and neural inhibition, we investigated the maturation of membrane electrical properties and inhibitory postsynaptic potentials (IPSPs). 


\section{Methods}

\section{Brain Slice Preparation}

Male or female gerbils, four to nineteen days old were decapitated by a scalpel and the brainstem caudal to the superior colliculus was dissected rapidly in oxygenated ice-cold artificial cerebral spinal fluid (ACSF). The ACSF in all experiments, unless otherwise noted, was composed of (in $\mathrm{mM}$ ) $\mathrm{NaCl}(124), \mathrm{KCl}$ (4), $\mathrm{KH}_{2} \mathrm{PO}_{4}$ (1), $\mathrm{NaHCO}_{3}$ (26), glucose (10), $\mathrm{CaCl}_{2}$ (3), $\mathrm{MgSO}_{4}$ (1). The cerebellum was removed by using iridectomy scissors so that the brainstem could be positioned more stably for sectioning. The brainstem was then glued (Loctite Corporation) to a petri dish, mounted against a piece of $4 \%$ agar under ice-cold ACSF and coronal sections $(200-250 \mu \mathrm{m})$ containing the SOC were taken by using a vibratome. Slices were transferred to a homemade holding chamber, which contained perfused, aerated ACSF $\left(95 \mathrm{O}_{2} / 5 \% \mathrm{CO}_{2}\right)$, at $37^{\circ} \mathrm{C}$ for at least one hour and then allowed to cool to room temperature. Slices were then transferred to a recording platform, attached to a fixed microscope stage and were constantly perfused with oxygenated ACSF at a flow rate of 2-3 mls per minute. The ACSF was maintained by feedback by a temperature controller (Warner Instruments) to $30^{\circ} \mathrm{C}$ during recording sessions. The $30^{\circ} \mathrm{C}$ temperature was chosen due to, for unknown reasons, the difficulty in obtaining recordings when the temperature was set to $37^{\circ} \mathrm{C}$ in our earliest experiments (data not shown). Slices were then viewed with a water immersion objective (40X) on a fixed staged, Axioskop microscope equipped with infrared/DIC optics. Video images were projected on a television monitor by using a CCD camera (MTI).

\section{Electrophysiological Recordings}

Recordings were made from principal cells of the MSO by using patch pipettes. Pipettes were pulled from $1.5 \mathrm{~mm}$ outer diameter borosilicate glass (World Precision Instruments) with a pipette puller (Brown Flaming or Mecanex) in 2-4 stages to tip diameters between 1-2.5 microns and filled with intracellular recording solution (in mM) Kgluconate (130), NaCl, EGTA (.2), HEPES (10), 
$\mathrm{MgCl}_{2}$ (2), NaATP (2). IPSPs were evoked by shocking the ipsilateral MNTB with parallel bipolar electrodes placed directly onto cells of the MNTB. The shock stimulus used was $\pm 1-100 \mathrm{~V}$ and $200 \mu \mathrm{s}$ in duration. The lowest voltage that elicited IPSPs was used in the experiment and the value varied among preparations. Data were collected at a sampling rate of $10-20 \mathrm{kHz}$ with a Multiclamp 700A amplifier (Axon instruments, Foster City, CA) controlled by Clampex 8.0 software (Axon instruments).

\section{Drug Application}

The shock-evoked postsynaptic potentials have both an excitatory and inhibitory component due to the activation of SBC axons that course in and around the MNTB en route to the MSO. The excitatory component is mediated by the neurotransmitter glutamate. These glutamatergic receptors were blocked by adding CNQX to block AMPA receptors and APV and MK801 to block NMDA receptors (10 $\mu \mathrm{M}$ each) in the ACSF.

\section{Data Analysis}

Data were analyzed offline with Clampfit software (Axon Instruments). The time constant, input resistance and spike properties of neurons were analyzed from voltage traces elicited by injecting a series of hyperpolarizing and depolarizing current pulses. The input resistance was measured by taking the slope of current-voltage (I-V) plots. The voltage used for I-V plots was taken from the steady state voltage near the end of the current stimulus. The membrane time constant was measured by single exponential fits of voltage responses to small rectangular, hyperpolarizing current injections.

IPSP durations were measured from the time when the voltage began to hyperpolarize to the time point when it recovered to the baseline voltage. IPSP recovery time constants were fit between the time of the maximum hyperpolarization to the end of the IPSP. Means and standard deviations were calculated for the time constant and input resistance for each age. Graphs are plotted as means \pm standard deviation. Statistical significance was determined by using analyses of variance (ANOVA) for between group comparisons. 


\section{Results}

\section{Electrical properties of MSO neurons}

Recordings were made from a total of 146 neurons before hearing onset (PD 4-11) and 124 neurons after hearing onset (PD 12-19). We were able to achieve stable recordings of the voltage of MSO neurons in response to hyperpolarizing and depolarizing current pulses injected through the recording pipette at all ages studied. The responses to current injections allowed us to classify the pattern of spike generation and to determine resting membrane potential, input resistance and membrane time constant. There were two distinct firing patterns elicited from depolarizing current injections, termed phasic and tonic. Cells that generated 1-3 action potentials at the onset of current injection and did not generate any more action potentials were classified as phasic. Cells that generated repetitive action potentials throughout the duration of the depolarizing current injection were classified as tonic. We qualitatively assessed that action potentials became shorter in duration as the age of the animal increased for both phasic and tonic cells (Figure 1).

Upon hyperpolarizing current injection, older neurons exhibited a voltage "sag" back toward the resting membrane potential after the peak voltage deflection. Voltage sags were found in both phasic and tonic neurons. Examples of voltage recordings of phasic and tonic cells at 5,10 and 15 days are shown in Figure 1.

The proportion of cells classified as tonic or phasic changed with age. During the first week of postnatal life (PD 4-7), 66\% (29 out of 44 cells) of the cells responded with a phasic firing pattern, and $34 \%$ (15 out of 44 cells) responded tonically. After PD 7 , the percentage of cells that responded with a phasic firing pattern steadily increased to $70 \%$ (158 out of 226 cells), whereas the percentage of cells with tonic responses fell to $30 \%$ (68 out of 226 cells). The numbers of tonic and phasic cells recorded at each age are plotted in Figure 2.

We calculated the input resistance of the cell from slopes of currentvoltage (I-V) plots elicited from rectangular depolarizing and hyperpolarizing current injections (see insets in Figure 1). Phasic neurons after PD11 
demonstrated steeper IV relationships than older phasic neurons, indicating a higher input resistance. We found that the input resistance of phasic cells recorded before PD 12 was on average $205 \mathrm{M} \Omega(n=59)$, but decreased to 56 $M \Omega$ in cells recorded after PD $11(\mathrm{n}=79)$ and were statistically different $(p<0.0001$; Figure 3$)$. The average input resistance of tonic cells decreased from $267 \pm 36 \mathrm{M} \Omega$ (PD 4-11; $\mathrm{n}=47$ ) to $175 \pm 65 \mathrm{M} \Omega$ (PD 12-19; $\mathrm{n}=28 ; P<0.01$; Figure $3)$.

The membrane time constant of phasic neurons ranged from $23.4 \mathrm{~ms}$ (PD 8) to $1.2 \mathrm{~ms}$ (PD 19). The average membrane time constant of phasic cells became faster with age and decreased from $16.8 \pm 4.7 \mathrm{~ms}$ (PD 3-11) to $2.3 \pm 0.9$ ms (PD 12-19); $\mathrm{n}=78$ and 86 , respectively $p<0.0001$ ). The average membrane time constant of tonic cells however, declined from $17.7 \pm 3.5$ (PD 3-11) to $12.4 \pm$ $3.8 \mathrm{~ms}(p<0.03$; PD 12-19). Plots of membrane time constant versus age for phasic and tonic cells are shown in Figure 4.

We measured the resting membrane potential (RMP) of the cell as the voltage recorded when the seal was first ruptured. There was no difference in the average resting membrane potential (RMP) between phasic and tonic neurons (phasic cells $=-45.7 \mathrm{mV} \pm 5.8 \mathrm{mV}$; tonic cells $=-47.7 \pm 4.7 \mathrm{mV}$ ) and this parameter did not vary with age (average RMP $=-44.1 \mathrm{mV}(\mathrm{PD} 3-11)$ and $46.1 \mathrm{mV}$ (PD11-19); $p=0.25$; Figure 5). These voltages are not corrected for a calculated liquid junction potential of $15 \mathrm{mV}$ (Clampex, Axon Instruments).

\section{Developmental changes in evoked IPSPs}

IPSPs were elicited by stimulation of the ipsilateral MNTB with shocks from bipolar electrodes. Prior to the first week of life, there was a decreased ability to evoke IPSPs in the MSO. We therefore report only on the development of IPSPs after the first week of life (PDs 7-19).

In PD 7-11 neurons, IPSPs tended to be relatively long and could be over $50 \mathrm{~ms}$ in duration in some recordings (Figure 6A). The duration of IPSPs tended to decrease with age of the animal, and could be as short as $5 \mathrm{msec}$ at PD 15 
(Figure 6A). The average duration of IPSPs prior to PD 12 was $23 \pm 8 \mathrm{~ms}$. After PD 11 the average IPSP duration was $7 \pm 1 \mathrm{~ms}$ (Figure 6B).

Another measure of the kinetics of the IPSPs is the speed of recovery to the baseline. We made measurements of the time constant of recovery of IPSPS (Figure 6) and found that the greatest change in IPSP recovery time constant that occurred with age was at PD 11. Prior to PD 11, the average IPSP recovery time constant was $29.6 \pm 7 \mathrm{~ms}(n=13)$, whereas after PD 11 the average IPSP recovery time constant decreased to $3.0 \pm 2 \mathrm{~ms}(n=63 ; p<0.0001$; Figure $5 \mathrm{C})$. 


\section{Discussion}

In summary, we made current clamp recordings from principal cells of the MSO in early postnatal gerbils and found that there are two distinctive cell types in response to current injections, termed phasic and tonic. Our results indicate that there is a much higher proportion of phasic cells versus tonic cells in PD 714 animals. We also found that the resting membrane potential did not change with age, but the input resistance and membrane time constant of phasic cells significantly decrease after the onset of hearing. Therefore, we conclude that most cells of the MSO after the onset of hearing, and likely into adulthood, are phasic cells, which have a low input resistance and a fast membrane time constant. Due to the difficulty in recording from the MSO beyond the second week of life we are unable to draw conclusions regarding the membrane electrical properties of MSO cells beyond PD 15.

\section{Response type}

Studies from other investigators indicate that the phasic response is probably due to one or more potassium conductances activated beyond resting membrane potential (Smith 1995; Manis and Marx 1991). Because of the negative reversal potential of potassium, an increased conductance is proposed to limit the ability of the cell to generate repetitive action potentials. The role of potassium channels in generating the response types of MSO cells will be considered in Chapter 5.

An argument could be made that that the differences recorded with age could be the result of differences in slice viability between younger and older animals. We conclude that this is not the case, because the resting membrane potential did not change with age and stable recordings could be made in some neurons for over an hour at all ages studied. Phasic responses could also be caused by damage of the cell by the recording electrode and effectively decreasing the resistance of the cell by making the cell "leaky" to ions. Since the resting membrane potential between phasic and tonic cells did not differ and cells 
generated brief, (1-2 ms) overshooting action potentials, we suggest that the response type was not due to cellular damage.

\section{Input resistance and time constant}

The input resistance of MSO cells and time constant of the membrane both decrease with age. The most significant decline was observed after 11 days of age. The membrane time constant is determined by the voltage dependence, kinetics and density of ion channels in the membrane of MSO cells. The time constant of the membrane is the product of the resistance of the membrane multiplied by the specific membrane capacitance. We find that for phasic cells the decline in total input resistance is not sufficient to explain the decline in the membrane time constant. The greater decrease in the membrane time constant is most likely due to a decrease in the overall cell surface area, thereby decreasing the total input capacitance. A decrease in cell surface area could result from a decrease in cell body size or from pruning of dendritic branches with age. These results are in contrast to previous studies, where in cells of the nucleus laminaris, the avian homologue of the MSO, a similar decrease in membrane time constant between embryonic and post-hatch chicks was observed (from $18.4 \mathrm{~ms}$ to $3.2 \mathrm{~ms}$ ) but no changes in the input capacitance were observed with age (Kuba et al. 2002).

\section{Synaptic transmission from the MNTB to MSO}

We reliably evoked IPSPs in the MSO from shocks of bipolar electrodes placed directly onto cells of the MNTB in PD 7-19 neurons. The inability to evoke IPSPs in younger animals may be the result of shaving the afferent input from the MNTB during the slicing procedure. Investigators who studied the development of inhibitory currents in the MSO had similar difficulties, where only 10 out of the reported 133 IPSCs were recorded before PD 8 (Smith et al. 2000).

The increased speed and duration of IPSPs may result from three mechanisms. First, the cell's input impedance and a derived measure, the time constant, shape postsynaptic potentials. Decreases in these parameters shorten the length of IPSPs. We found that after the onset of hearing the membrane time 
constant and impedance are low, which correlated well with a decrease in the durations of IPSPs. The high correlation coefficient indicates that the decrease in the IPSP time constant was almost completely dependent upon the decrease in the membrane time constant.

Second, the IPSP shape is determined in part by the inhibitory postsynaptic current (IPSC). In a study of IPSCs in PD 3-14 in the MSO of the rat, IPSCs were greatly accelerated after PD 11 (Smith et al. 2000). In younger neurons IPSCs were fit with a two component decay; the fast component decayed with a time constant of $7.8 \mathrm{~ms}$ and the slow component decayed with a time constant of $38 \mathrm{~ms}$. After PD 11 the time constant of the fast component was $3.9 \mathrm{~ms}$ and the slow component became negligible. The results of these studies correlate well with our findings that IPSPs had a time constant of recovery of about $3.0 \mathrm{~ms}$ a difference of only about $25 \%$ which is most likely due to species differences and experimental conditions.

Third, the movement of inhibitory inputs to the cell body during development (Kapfer et al. 2002) could also decrease the duration of IPSPs. We discuss in the next chapter that the placement of excitatory inputs on dendrites can affect the duration of EPSPs.

Overall, we conclude that the decrease in the membrane time constant is the main factor in the acceleration of IPSPs between younger and older animals. After PD 12 the decrease in the IPSP time constant may result from the increased speed of the IPSC and refinements of inputs to the cell body. 

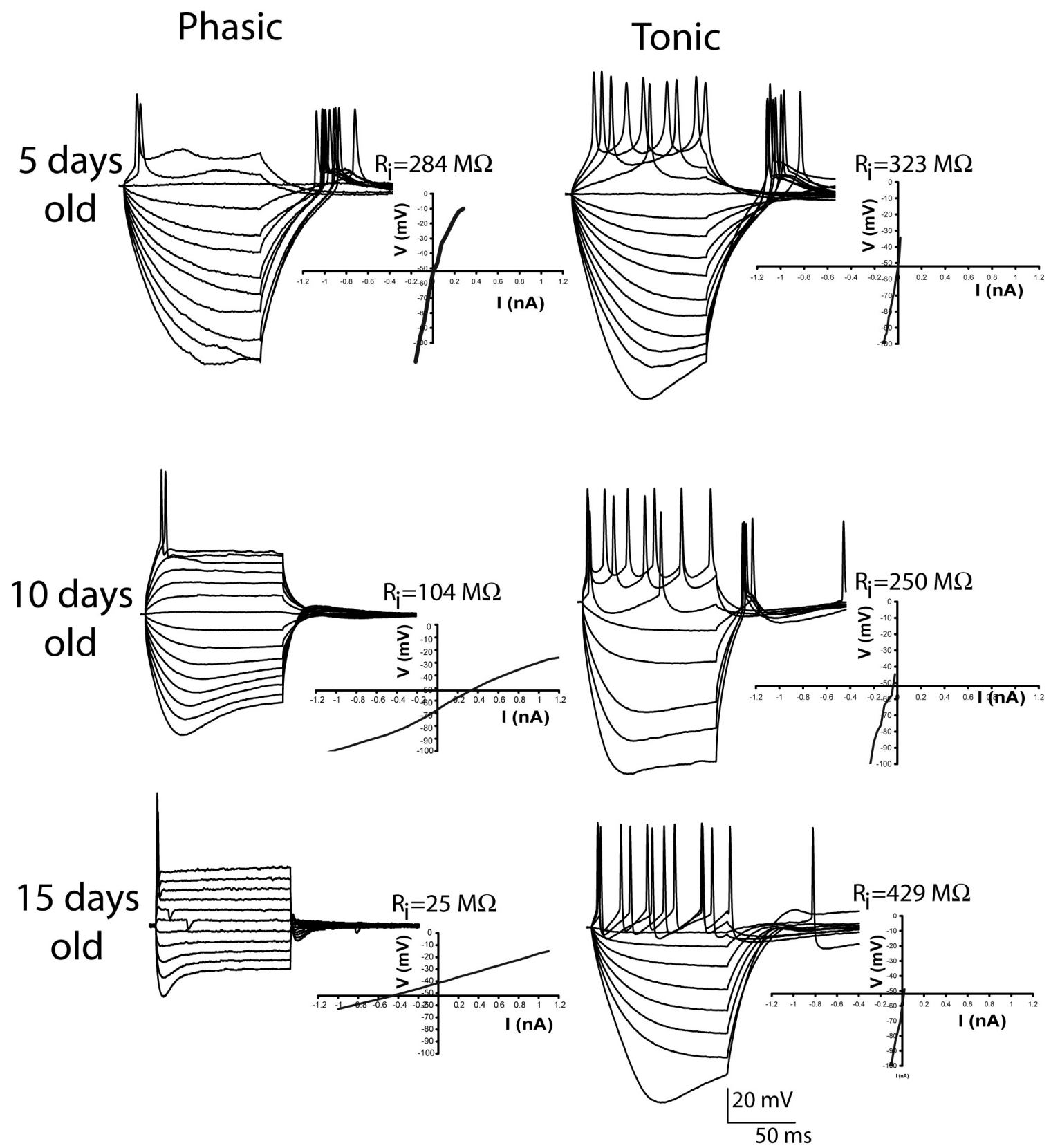

Figure 1. Representative records of membrane properties and firing patterns of MSO cells at 5,10 and 15 days.

Injections of depolarizing square current pulse injections resulted in either a few spikes at current onset (Phasic) or repetitive generation of action potentials (Tonic). Phasic neurons recorded from young animals (PD 3-11) had slow 
membrane time constants, whereas in older neurons the membrane time constant became dramatically faster. Note that the duration to reach steady state voltage is greatly reduced in older animals. A decrease in the membrane time constant of tonic cells was not observed. The input resistance (Ri) as measured from the slopes of I-V curves (inset to right of each series of traces) was reduced after PD 11 in phasic neurons; the $\mathrm{Ri}$ of tonic neurons remained elevated throughout the time period studied. Note that we made no adjustments to the resting membrane potential by an injection of any holding currents, therefore the baseline accurately reflects the resting membrane potential of the cell. 


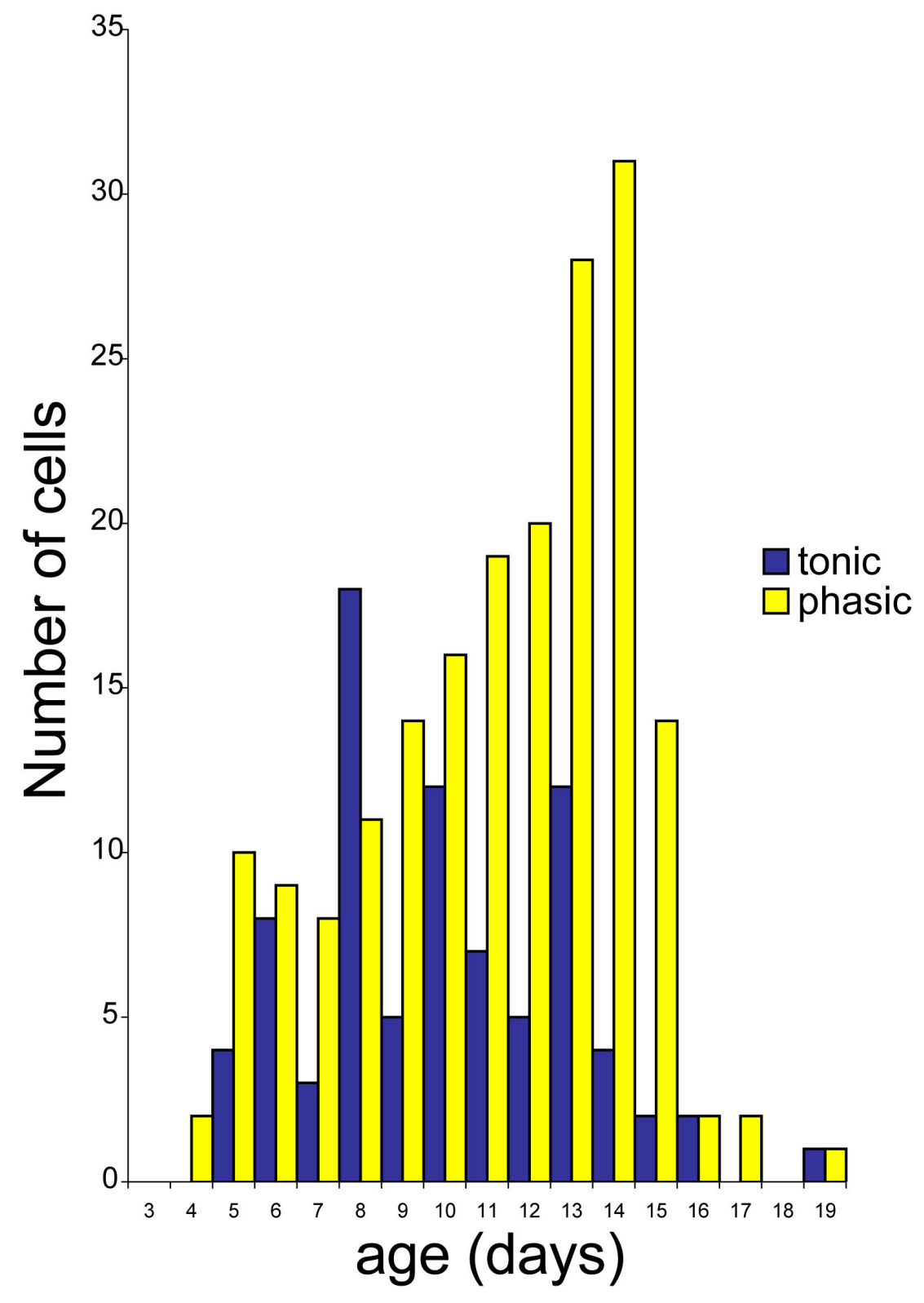

Figure 2. Distribution of cell types during the first 19 days of postnatal life.

After the first week of life, there was a dramatic increase in the number of phasic cells compared to tonic cells. 

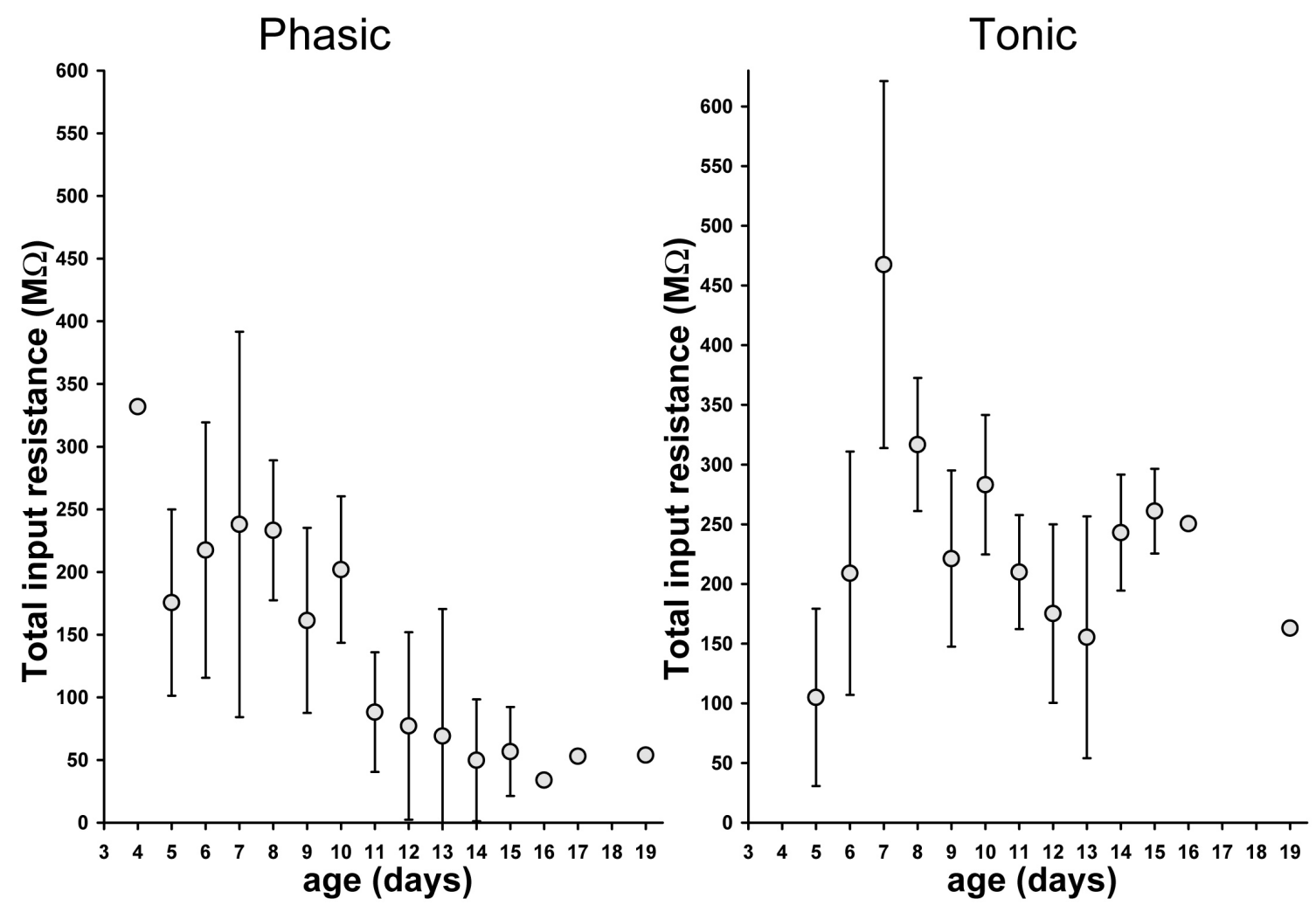

Figure 3. The input resistance of phasic cells and tonic cells decreased during the second week of life. 

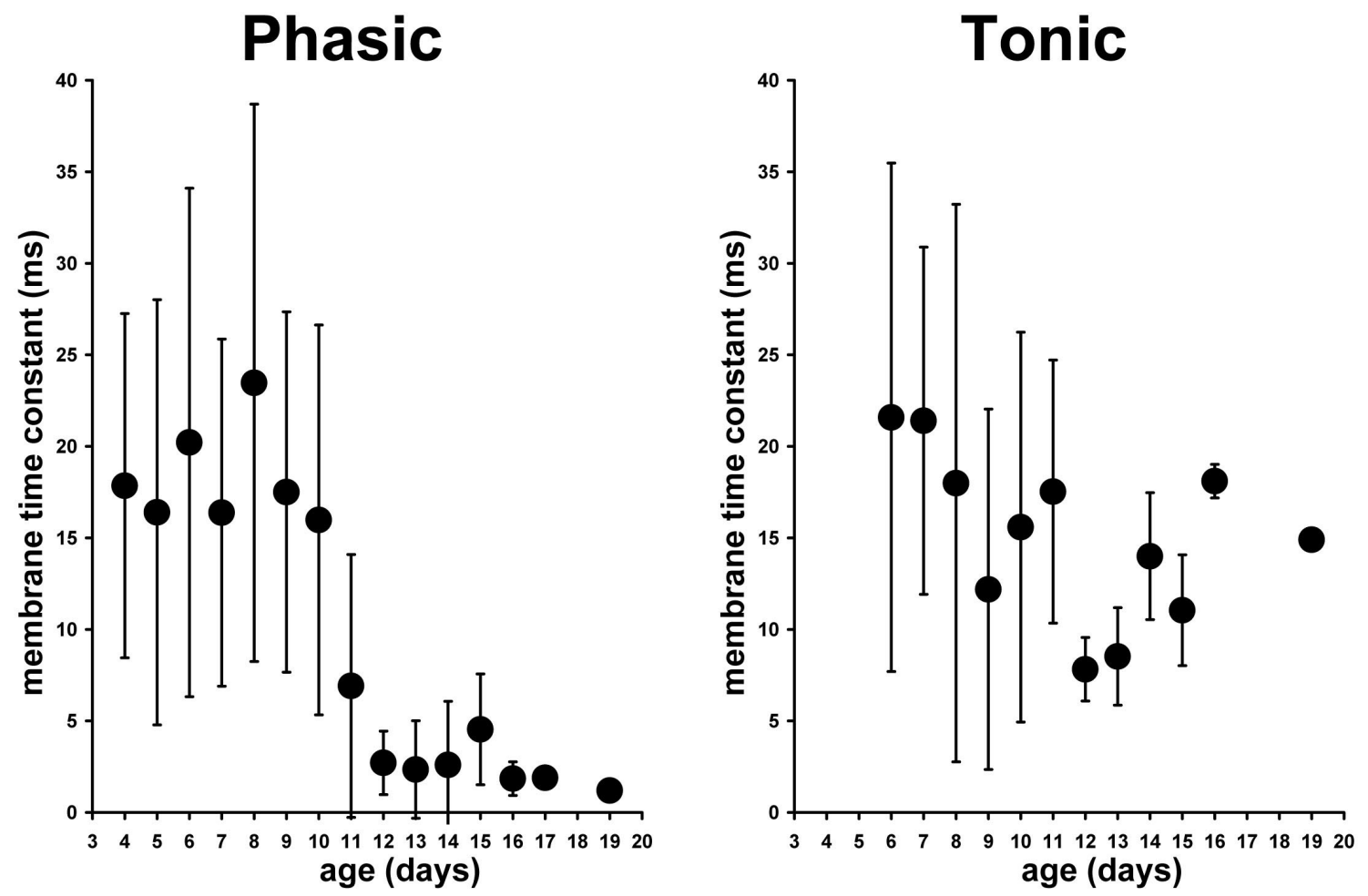

Figure 4. Mean values of membrane time constant of phasic and tonic cells versus age of the animal.

The membrane time constant of phasic cells decreased after PD 12. There was no obvious decrease in the membrane time constant with age for tonically active cells. 


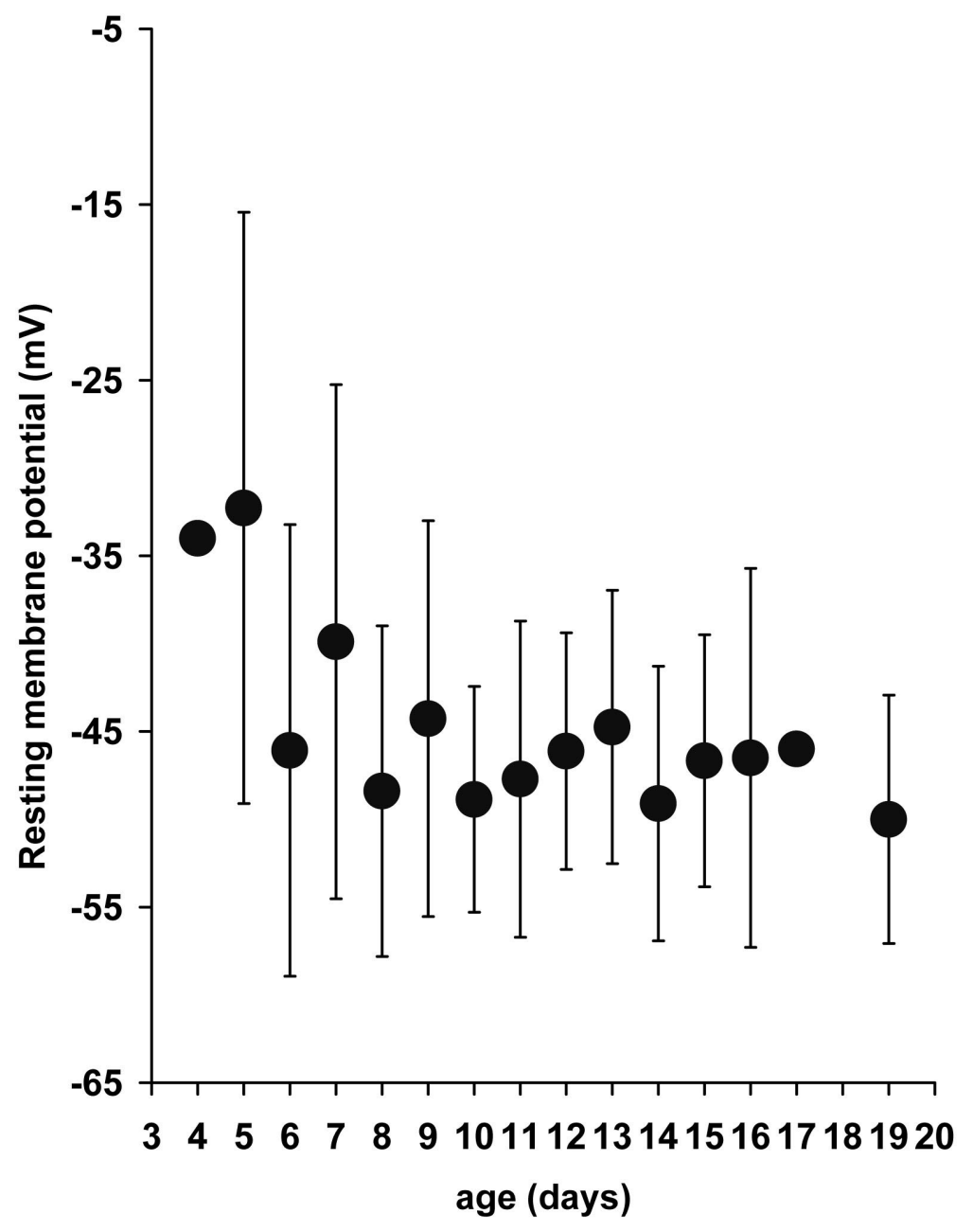

Figure 5. Mean values of the resting membrane potential at each of the different ages studied.

The average resting membrane potential did not vary with increasing age. 

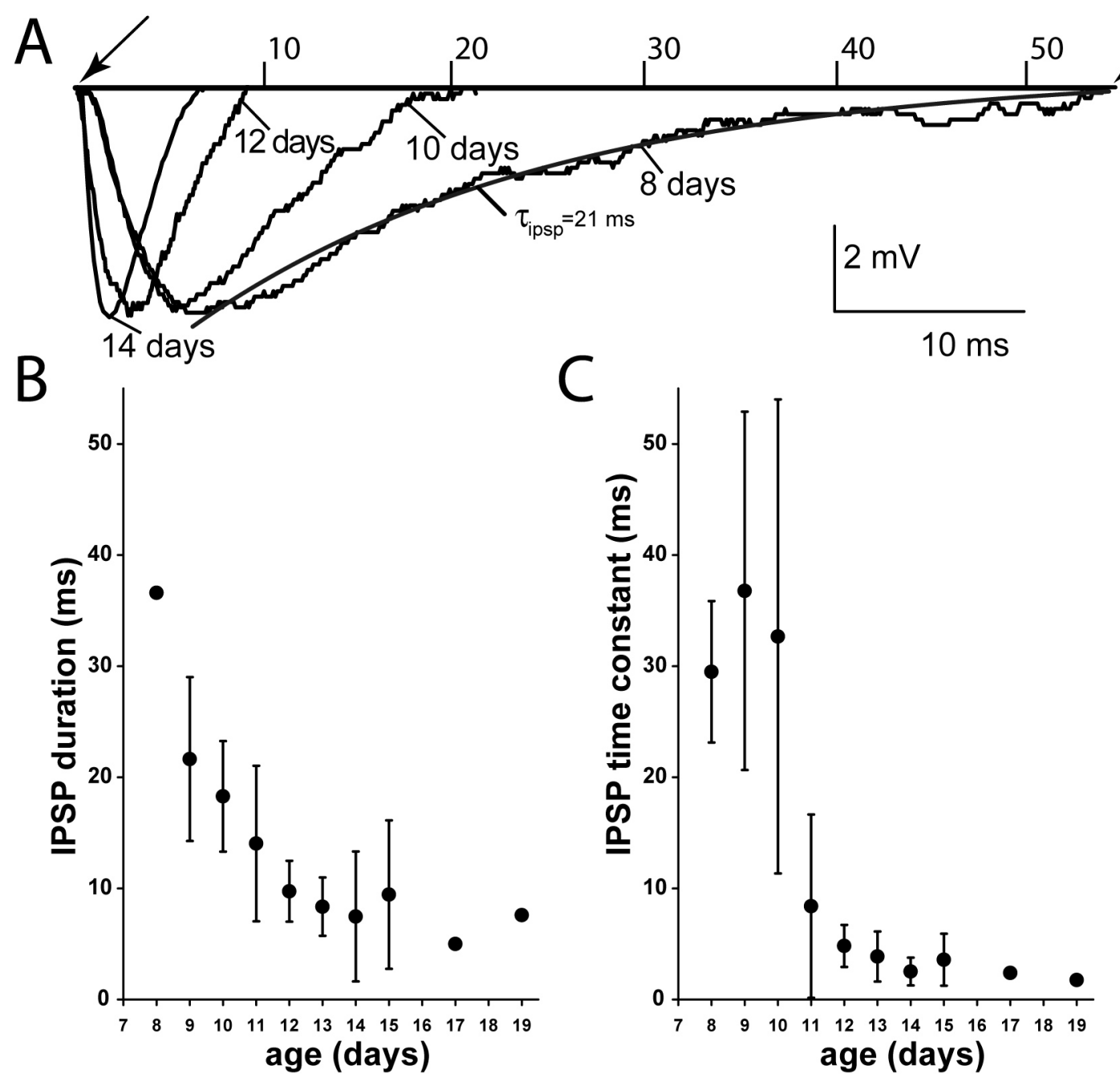

Figure 6. Temporal characteristics of IPSPs versus age.

A. Examples of IPSPs recorded from PD 8, 10, 12, and 14 animals. Arrows mark the time points used to measure the duration of the IPSP. An example of an exponential fit is shown in the PD 8 IPSP.

B. The duration of IPSPs steadily declined with increasing age of the animal. C. The time constant of recovery of IPSPs was longer in younger animals, but dramatically decreased after PD 12. 


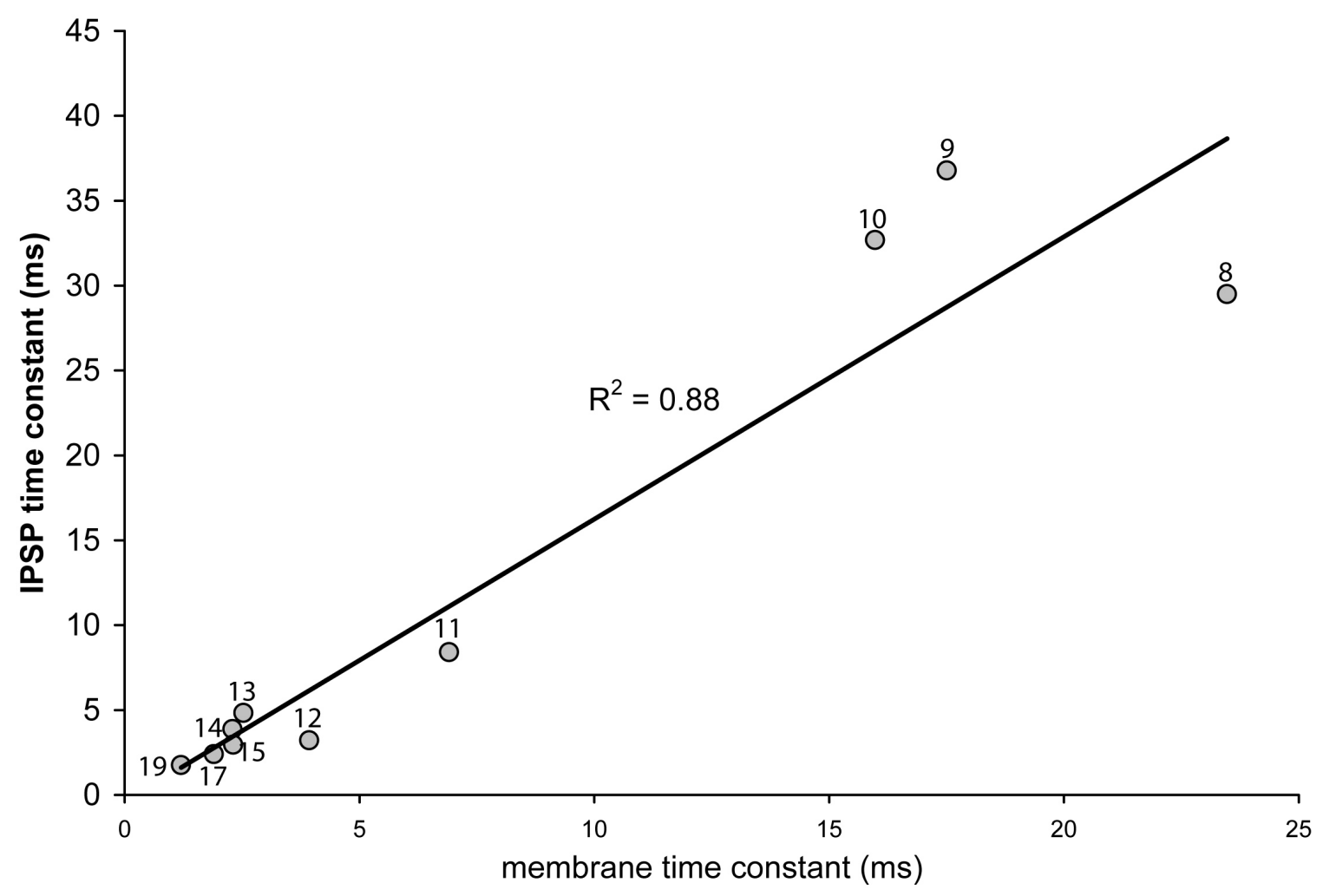

Figure 7. Plot of average time constant of recovery of IPSPs versus the average membrane time constant.

The time constant of recovery of IPSPs between PDs 8-19 were strongly correlated $\left(R^{2}=0.88\right)$ with the observed decrease in the membrane time constant over the entire age range studied. The PD is labeled beside each data point. 
Chapter 4: Characterization of a model MSO neuron to generate simulated auditory stimuli for use in brain slice experiments. 


\section{Introduction}

The roles of voltage-gated potassium channels and synaptic inhibition in the ability of MSO cells to code ITDs are unknown. In Study 3 we aimed to dissect the contributions of these cellular mechanisms by use of in vitro MSO cell recordings coupled with injection of simulated auditory stimuli through the recording pipette. This approach is advantageous because it enables the investigator to study the role of intrinsic membrane properties in ITD coding in the absence of confounding in vivo synaptic processes. These processes include depletion of synaptic vesicles during sustained stimuli (synaptic depression) and the temporal variations in neurotransmission (also called synaptic jitter) through the synapse. Most important, these methods allow for the study of excitatory and inhibitory influences on time-critical binaural computations both separately and together.

We employed a technique to simulate synaptic inputs that utilized a morphological reconstruction of a real MSO cell that was imported into modeling software. In the previous study, we labeled MSO cells and then made morphological observations and measurements of the dendritic fields. The cell selected for the modeling synaptic inputs fell in the mid-range of the measured dendritic lengths for our entire population of labeled MSO neurons. The model cell was then imported into a neuronal simulation program (NEURON) and used to generate stimuli designed to mimic the net synaptic current generated in the soma of a MSO cell. We simulated conditions where synaptic inputs driven from each ear arrive in coincidence and then simulated conditions of non-coincident inputs. The results of the simulation (composite EPSP waveforms recorded from the model cell soma) were used to stimulate living MSO cells in a brain slice preparation. We coupled this technique with the electrical activation of inhibitory inputs while recording from MSO cells in brain slices and, in separate experiments, pharmacological block of specific voltage-gated potassium channels (Chapter 5). 


\section{Methods and Results}

\section{Morphology of MSO Cells-Selection of the Model Cell}

We labeled cells in Study 2 and used one of those cells to construct a model MSO cell used to generate stimuli for the study of ITD coding (Study 3 ). We analyzed the morphology of MSO cells in animals older than postnatal day 11 from Study 2, and then added cells to our database from recordings made during Study 3. The complete set of MSO neurons is described here. The morphology of MSO cells was revealed from labeling cells with markers included in the electrode filling solution. Pipettes (described in Chapter 3 ) were filled with Neurobiotin (1\%) and Lucifer Yellow (0.1\%) that were added to a standard intracellular recording solution (in mM) K-gluconate (130), $\mathrm{NaCl}_{4}$, EGTA (.2), HEPES (10), $\mathrm{MgCl}_{2}$ (2), NaATP (2). After the recording sessions (Study 2), slices were fixed in $4 \%$ paraformaldehyde and $0.1 \%$ glutaraldehyde in a $0.12 \mathrm{M}$ $\mathrm{NaPO}_{4}$ buffer solution overnight. Slices were then rinsed in $0.12 \mathrm{M} \mathrm{NaPO}_{4}$ solution and Lucifer yellow was revealed by using immunocytochemistry. Neurobiotin was revealed using the avidin-biotin complex reaction ( $A B C)$ intensified by the heavy metals nickel and cobalt. These reactions confirm the location of the recording site and reveal the morphology of the cell body, dendrites and axon. Slices were then mounted on slides using Cresyl gel, allowed to dry and coverslipped. Camera lucida drawings were made by using a $100 \mathrm{X}$ oil immersion lens on an Olympus microscope.

We quantified the dendritic arrangements emanating from MSO cell bodies as a first step in investigating the effects of varying the location of synaptic inputs in the model. We made measurements from 29 camera lucida drawings of MSO cells from animals that were at least 12 days of age. Examples of camera lucida drawings of the cells are shown in Figure 1. MSO cells typically had 1-2 thick primary dendrites (diameter 1-3 $\mu \mathrm{m}$; average equal to $2.4 \mu \mathrm{m}$ ) extending from both the medial and lateral segments of the cell soma thus giving a bipolar appearance. In 10 out of 29 labeled cells, one side had more than one primary dendrite. If a cell had two primary dendrites on one side, the cell 
typically did not have more than one primary dendrite on the other side. There were numerous branches off of the primary dendrites and most of the branches continued in the mediolateral direction and remained thick to near their terminus. Thin dendrites, which were typically branched, extended out of the ends of thick dendrites. The diameter of the thin dendrites ranged from 0.1 to $0.65 \mu \mathrm{m}$. In four cells, however, one of the primary dendrites gave rise to a branch directed dorsally (Figure 1 cells $\mathrm{C}$ and $\mathrm{E}$ ). In three cells, a fourth primary dendrite emerged from the cell body (Figure $1 \mathrm{H}$ ).

We measured the lengths of dendrites by importing images of camera lucida drawings into a Macintosh computer and tracing dendritic lengths by using the "Freehand" tool of NIH image. We measured and compared the dendritic lengths of only thick dendrites and compared medially to laterally oriented dendrites from our cells and from cells published in the literature (Russell and Moore 2002; Smith 1995). The measured dendritic lengths are listed in Table 1. We found in our sample that MSO cells (19 out of 29) were unequally weighted in their dendritic length distributions with a bias towards the lateral side. A scatter plot of the summed lengths of medial versus lateral dendrites is shown in Figure 2.

The cell chosen to model the effects of synaptic inputs had a complex set of thin, branching dendrites at the ends of its thick dendrites. The cell was selected because it contained the essential features of thick dendrites but also allowed us to more completely investigate placement of inputs onto smaller, branching dendrites. The cell fell into the mid range of summed dendritic lengths and had equal lengths of thick dendrites on each side. A cylindrical, compartmental model of the three-dimensional morphology of the MSO cell was made by using the program Neurolucida (Microbrightfield Inc.; model cell shown in Figure 1A). To construct the model cell, slides containing sections with labeled cells were placed under a 100X oil immersion objective on an Olympus microscope equipped with a motorized stage and Z-position readout. The image of the cell was captured by a video camera and viewed within Neurolucida on a computer screen. Each cylinder is determined by a length and diameter 
measurement made by the investigator. The length is measured by clicking several points along the dendritic tree. The diameter is determined by keeping Neurolucida's circular cursor the same width as the dendrite being traced. The Z-depth is automatically tracked as focus adjustments by the software. The reconstructed geometry was saved in a version 2 ASCII file format that is converted into a NEURON file (.hoc file format) by a third-party file converter CVAPP (Robert Cannon). Files containing the three-dimensional information of the cell were then imported into the neuronal simulation program NEURON (Carnevale and Hines; freely available on the web at http://neuron.duke.edu).

\section{Characterization of the Model MSO: Cell-Simulations in NEURON}

The goal of using NEURON was to generate stimuli that mimic the voltage response seen in the soma of MSO cells to different ITDs, and to use them as current injection stimuli through the recording pipette attached to a living MSO neuron. Before performing the in vitro experiments, we characterized the model cell response to simulated synaptic input. After importing the MSO cell geometry into NEURON a passive membrane model of the cell was created. A model with only passive membrane properties contains only a leakage conductance, which was set to a standard value of $.001 \mathrm{mho} / \mathrm{cm}^{2}$, and does not contain voltage-gated ion channels. The reversal potential of the leakage conductance was set to -70 $\mathrm{mV}$. We chose to use a model with only passive membrane properties in order to avoid making assumptions about types and regional distributions of ionic channels. The temperature of the simulation was set to $30^{\circ} \mathrm{C}$, which is the same temperature as the bath temperature in the in vitro brain slice experiments (Chapter 5). The axial resistance and capacitance of the membrane were set to typical values estimated from mammalian systems $\left(160 \mathrm{M} \Omega / \mathrm{cm}\right.$ and $1 \mu \mathrm{F} / \mathrm{cm}^{2}$, respectively (Jack 1983)). The parameters used in the model are listed in Table 2.

The excitatory inputs were modeled as a conductance change described by an alpha function. The synaptic current with an alpha function conductance is defined by: 


$$
I=g\left(V-V_{r}\right)
$$

Where:

$$
\begin{gathered}
I=\text { current, } g=\text { conductance, } V=\text { voltage, } V_{r}=\text { reversal voltage } \\
\qquad g=0 \text { for } t<\text { onset } \text { and assume onset at } \mathrm{t}=0 \\
\mathrm{~g}=\operatorname{gmax}^{*}(\mathrm{t}-\text { onset }) / \alpha^{*} \exp (-(\mathrm{t}-\text { onset }-\alpha) / \alpha)
\end{gathered}
$$

Where:

$$
\alpha=\text { time to peak of conductance change }
$$

\section{Effects of changing the time to peak $(\alpha)$ of the alpha function}

As a first step in a series of simulations to characterize the model cell, we injected a current described by an alpha function directly into the soma and recorded the voltage at the same site. The alpha function is a time-dependent and voltage-independent conductance change that rises to a peak value then decays more slowly than the rise to peak (Rall 1967). The alpha function has been used to model excitatory input to auditory brainstem cells (Banks and Sachs 1991; Rothman et al. 1993; Cai et al. 2000). The voltage measured in response to current injection is a solution of the cable equation for that compartment of the model (Carnevale and Hines). NEURON calculates voltage for each compartment of the model by injecting axial current from one compartment into the next compartment in the series. The voltage response of the cell to an excitatory current injection will herein be called an excitatory postsynaptic potential (EPSP). We demonstrated the effects of changing the time to peak $(\alpha)$ of the alpha-function and found that by decreasing $\alpha$, as expected, decreased the time to peak and the overall duration of EPSPs. Examples of EPSPs with different $\alpha$ 's are shown in Figure 3. The peak of the EPSP occurs later than the peak of the alpha function resulting from the delay in the charging of the membrane capacitance of the cell. In our remaining investigations of the model cell, we set the value of the alpha synapse to $2 \mathrm{~ms}$ as it resulted in EPSPs similar in duration to the EPSPs we recorded in vitro.

\section{Effects of synaptic location on the EPSP shape at the soma}

The location of synapses on dendrites can affect the amplitude, duration 
and onset time of EPSPs at the soma and affect spatial summation. Spatial summation occurs when two subthreshold events occur at different points along the dendritic membrane and summate beyond threshold and an action potential is generated. To determine the effects of moving the synaptic location on the EPSP, we systematically tested four locations for excitatory current injections in both the medial and lateral dendrites. Six synaptic inputs were placed onto thick dendrites ( 3 on each side) and two synaptic inputs ( 1 per side) were placed on distal, thin dendritic branches. The placements of synaptic inputs onto the model cell and results from these simulations are shown in Figure 4. The alpha function was set to $2.0 \mathrm{~ms}$. When the current injections were moved more distally the maximum amplitude of the EPSP declined. We compared the decay in voltage between sides and found that medial dendrites caused only a slightly greater decay in EPSP amplitude per micron than the lateral dendrites. The EPSP declined $0.1 \mathrm{mV}$ every $18 \mu \mathrm{m}$ in the medial dendrites, whereas the lateral dendrites declined $0.1 \mathrm{mV}$ every $22 \mu \mathrm{m}$ (Figure 4B and 4C).

In addition to amplitude, we measured the time at which the EPSP peaked in the soma from the eight synaptic locations shown in Figure 4A. We found that for both the medial and lateral dendrites the peak of the EPSP was delayed as the synaptic locations moved more distally. The peak of the EPSP was delayed $100 \mu$ secs for every $12 \mu \mathrm{m}$ in medially oriented dendrites and was delayed 100 $\mu$ secs every $13 \mu \mathrm{m}$ in laterally oriented dendrites. A graph of the peak of the EPSP plotted as a function of distance is depicted in Figure 4D. We conclude that the placement of synaptic input on dendrites could affect spatial summation of postsynaptic potentials. Placement of inputs is especially important in the gerbil MSO neurons, given that the head size of the gerbil is small, and sound waves at the two ears only produce a maximum ITD of $100 \mu$ secs.

As an unbiased measure of the duration of EPSPs evoked from different synaptic locations, we measured the duration of the EPSP as the time from the peak of the EPSP to the time it decayed two time constants from the peak of the EPSP (Figure 4E). We found that when we moved the synaptic injection site more distally the duration of the EPSP, by using our measure, increased by less 
than $2 \%$.

\section{Structural variations of the model cell}

The differences in the lengths of the dendrites seen in the MSO column could act to filter some of the current reaching the soma. To compare the model cell and cells with longer dendrites, we tested whether increasing the lengths of the two main primary dendrites significantly altered the response seen in the soma of the model cell. The dendritic lengths of the medially and laterally oriented dendrites were increased by $50 \mu \mathrm{m}$ by altering the dendritic lengths in the "Cell Builder" window in NEURON. The increased dendritic lengths represented a $25 \%$ increase in the total dendritic length of the cell. When we injected current into location number 3 on each side, the increased dendritic lengths caused an attenuation of the EPSP recorded in the soma. The peak of the EPSP of the cell with longer dendrites was decreased by $0.5 \mathrm{mV}$ (Figure 5). The time to peak of the EPSP was delayed by only $7 \mu \mathrm{s}$, and the duration measured at a two time constant decay from the peak increased by $17 \mu \mathrm{s}$. We conclude that changes of dendritic lengths of MSO cells of less than $50 \%$ have a significant effect on the amplitudes of EPSPs but have a relatively small effect on the time to peak of the waveform.

The effect of morphology on the shape of the EPSPs was further tested by removing either the medial or lateral dendritic fields. The dendritic fields were removed by using the "Remove Section" feature in the Cell Builder window of NEURON. Excitatory current was injected into the remaining dendrite and voltage was recorded in the soma. When either the medial or lateral dendritic fields were amputated, the amplitude of the EPSP was increased compared to the amplitude recorded in the complete model. The amplitude of the EPSP when the medial dendritic field was removed increased from 2.7 to $3.0 \mathrm{mV}$ and the time to peak decreased by $100 \mu \mathrm{s}$. When the lateral dendrites were removed the amplitude of the EPSP recorded in the soma increased from 1.48 to $1.98 \mathrm{mV}$ and the time to peak decreased by $300 \mu \mathrm{s}$. The increase in the amplitude of the response is probably due to the removal of the large current sinks that the 
dendrites pose on the cell. The importance of dendritic fields in auditory coincidence detection has been tested by other investigators (Agmon-Snir, 1998). In their neuronal simulations, they found that the opposite dendrite acts as a current sink to synaptic input on the opposite dendritic pole. Thus, if two simultaneous inputs arrive on the same dendrite the threshold for action potential is greater than if they occurred on opposite dendrites.

\section{Addition of multiple synapses}

We simulated the effects of adding multiple synapses (three to each side in different locations) to the model MSO cell at approximately the same electrotonic distance. Addition of synapses only increased the amplitude of the voltage response and did not significantly change the time course of the synaptic events (Figure 6). Dividing the conductance for each synapse by the total number of synapses generates a voltage response nearly equal to the response seen with only one synapse. Therefore we conclude that our simulations of multiple, simultaneous arriving synaptic input at equidistant electrotonic distances can be achieved by the scaling of EPSPs.

\section{Generation of Simulated Auditory Stimuli}

Sounds originating away from the midline introduce a difference in the arrival time of a sound between the two ears (interaural temporal delay or ITD). The delay is transferred to the arrival times of inputs onto the medial and laterally oriented dendrites of MSO cells. We simulated ITDs by delaying input onto the dendrites and recording EPSPs in the soma of the model cell. The recorded EPSPs were later used as current injection stimuli through the recording pipette into living MSO cells (described elsewhere). We placed three synaptic inputs onto midway points of second order dendrites on both the medial and lateral dendrites (locations numbered in Figure 6 Top). The synaptic current injection was the same as described above, with the alpha function set to a value of $2 \mathrm{~ms}$. We adjusted for variations in EPSP amplitudes of each side, to make each side equally weighted by adjusting the conductance of the excitatory synaptic current injection. ITDs were simulated by delaying the activation of one dendritic field 
relative to the other in increments of $100 \mu \mathrm{sec}$ to a value of 1.5 msecs. We found that increasing the simulated ITD produces a biphasic EPSP and causes a decrease in the amplitude of EPSPs with increasing ITD.

The results from our simulations produced EPSPs that were long in duration (greater than $20 \mathrm{~ms}$ ). Based upon these results and results from our studies of inhibition (Chapter 5), we decided to scale the EPSPs so that they were shorter in duration. We scaled the EPSPs, by decreasing the $\alpha$ function so that the EPSP was completed within $10 \mathrm{~ms}$ and therefore can operate at a frequency of $100 \mathrm{~Hz}$. The scaling was done because we ultimately wanted to study the interactions between excitatory and inhibitory inputs at equally matched frequencies $(100 \mathrm{~Hz})$. We chose this frequency because inhibitory post-synaptic potentials recorded from MSO cells begin to fail above $100 \mathrm{~Hz}$. The simulated ITD current injection stimuli that were used in the in vitro experiments are shown in Figure 7.

We used a morphologically realistic cell to characterize the passive electrical properties of a MSO cell. For the most part, the model cell responses to simulated excitatory current injection were in accordance with other modeling studies (Agmon-Snir, 2002). Our most interesting result however, is the finding that by moving excitatory synapses more distally the peaks of the EPSPs were delayed $100 \mu$ secs for approximately every $13 \mu \mathrm{m}$. The relatively small differences in the placement of synaptic inputs greatly affected the timing of the peak of EPSPs, which can have profound effects on the spatial summation of inputs and therefore the acuity to which sounds can be localized. The sensitivity of MSO cells to changes in EPSP shapes are explored and discussed in the following two chapters. 


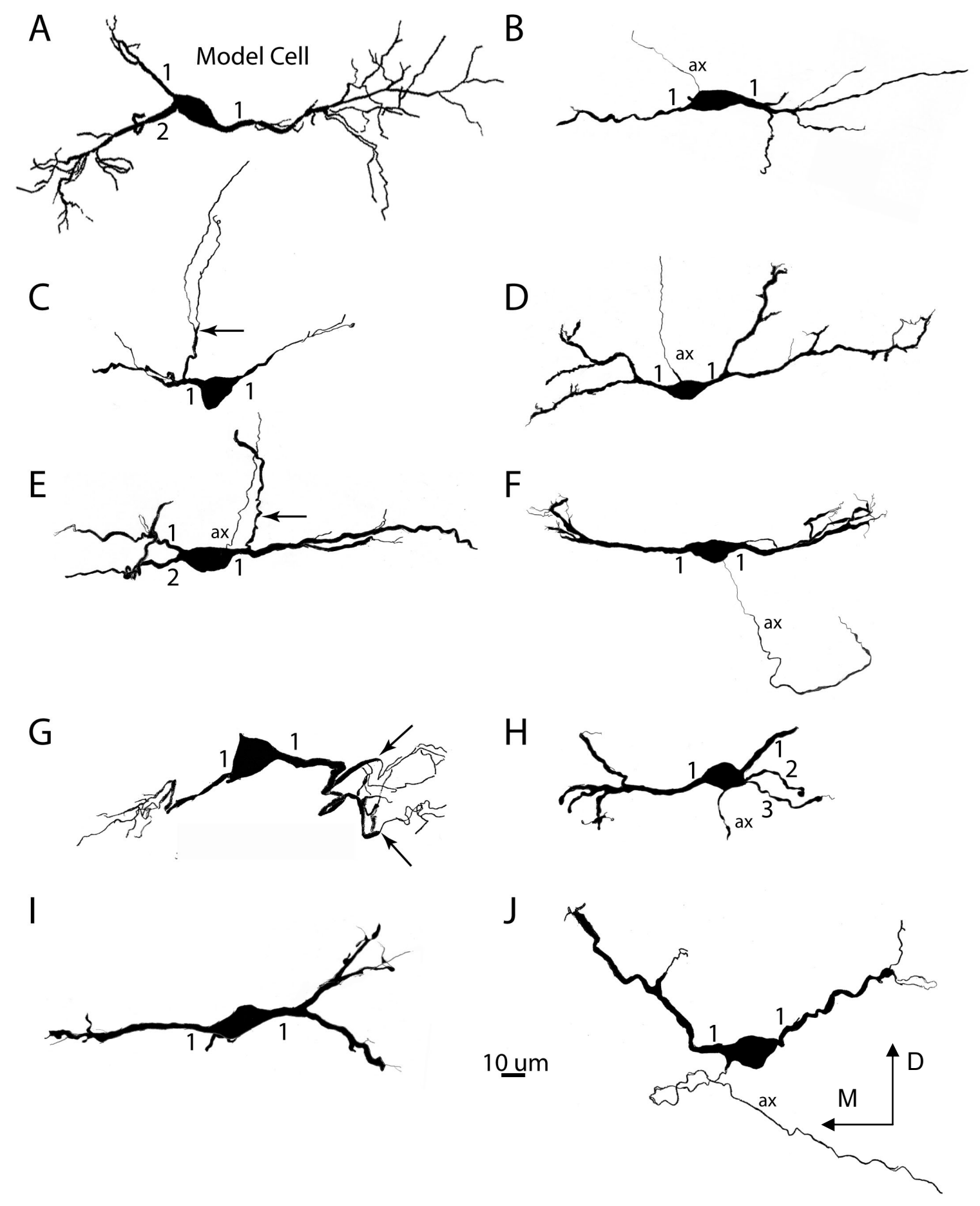

Figure 1. Cameral lucida drawings of ten intracellularly labeled MSO cells. 
MSO cells were labeled by using a patch pipette that contained Neurobiotin and Lucifer yellow. MSO cells are bipolar in appearance having 1-3 primary dendrites per side (numbered in each drawing) directed in the mediolateral direction. In some cases, primary dendrites give rise to a dendritic branch that coursed dorsally (arrows label dorsal dendrites in cells $\mathrm{C}$ and $\mathrm{E}$ ). Axons typically originate from the cell body. (labeled ax in cells $D, E, F, H$, and J). ax, axon D, dorsal. M, medial. 


\begin{tabular}{|c|c|c|c|c|c|c|c|c|}
\hline Cell & $\begin{array}{l}\text { Number of } \\
\text { medial } \\
\text { primary } \\
\text { dendrites }\end{array}$ & $\begin{array}{l}\text { Number of } \\
\text { lateral } \\
\text { primary } \\
\text { dendrites }\end{array}$ & $\begin{array}{c}\text { Medial } \\
\text { primary } \\
\text { dendrite } \\
\text { lengths } \\
(\mu \mathrm{m})\end{array}$ & $\begin{array}{l}\text { Lateral } \\
\text { primary } \\
\text { dendrite } \\
\text { lengths } \\
(\mu \mathrm{m})\end{array}$ & $\begin{array}{l}\text { Summed } \\
\text { lengths of } \\
\text { medial } \\
\text { dendrites } \\
(\mu \mathrm{m})\end{array}$ & $\begin{array}{c}\text { Summed } \\
\text { lengths of } \\
\text { lateral } \\
\text { dendrites } \\
(\mu \mathrm{m})\end{array}$ & $\begin{array}{l}\text { Medial/ } \\
\text { Lateral }\end{array}$ & Axon \\
\hline Model & 2 & 1 & 14,19 & 32 & 126 & 124 & 1.01 & none \\
\hline $6 / 12 / 01$ & 2 & 1 & 89,75 & 8 & 164 & 303 & .54 & cell body \\
\hline $7 / 25 / 02 \mathrm{~A}$ & 1 & 1 & 72 & 55 & 72 & 119 & 0.61 & cell body \\
\hline $7 / 25 / 02$ B & 2 & 1 & 48,25 & 46 & 152 & 181 & 0.84 & cell body \\
\hline $7 / 13 / 01$ & N/A & 2 & $\mathrm{~N} / \mathrm{A}$ & 46,55 & N/A & 115 & N/A & none \\
\hline $6 / 11 / 01 \mathrm{~A}$ & 1 & 1 & 48 & 65 & 48 & 72 & 0.67 & none \\
\hline $6 / 11 / 01 B$ & 1 & 1 & 9 & 43 & 53 & 43 & 1.23 & none \\
\hline 6/19/02 & 1 & 3 & 54 & $49,50,61$ & 199 & 162 & 1.23 & cell body \\
\hline 7/30/01 & 1 & 2 & 14 & 38,61 & 132 & 151 & 0.87 & $\begin{array}{l}\text { lateral } \\
\text { dendrite }\end{array}$ \\
\hline $4 / 10 / 01$ & 1 & 1 & 36 & 32 & 97 & 143 & 0.68 & cell body \\
\hline $6 / 13 / 01 \mathrm{~A}$ & 1 & 1 & 13 & 90 & 116 & 90 & 1.29 & cell body \\
\hline 6/13/01 B & 1 & $\begin{array}{l}1 \text { plus } 1 \\
\text { dorsal }\end{array}$ & cut & cut & 204 & $\mathrm{~N} / \mathrm{A}$ & $\mathrm{N} / \mathrm{A}$ & none \\
\hline $8 / 6 / 01$ & 1 & 1 & 74 & 12 & 74 & 116 & .63 & none \\
\hline $6 / 18 / 02 \mathrm{~A}$ & 1 & 1 & 46 & 11 & 46 & 69 & .66 & cell body \\
\hline $6 / 18 / 02$ B & 1 & 1 & 54 & 8 & 54 & 138 & .39 & cell body \\
\hline $6 / 24 / 02$ & 2 & 1 & 38,86 & 19 & 124 & 182 & .67 & cell body \\
\hline $6 / 30 / 02$ & 1 & 1 & 71 & 41 & 151 & 202 & .74 & cell body \\
\hline $7 / 22 / 02 \mathrm{~A}$ & 1 & 1 & 84 & 15 & 84 & 281 & .29 & cell body \\
\hline $7 / 22 / 02$ B & 1 & 1 & 56 & 20 & 83 & 150 & .55 & $\begin{array}{l}\text { lateral } \\
\text { dendrite }\end{array}$ \\
\hline $7 / 22 / 02 \mathrm{C}$ & 1 & 1 & 21 & 18 & 202 & 265 & .76 & cell body \\
\hline $7 / 22 / 02 \mathrm{D}$ & 2 & $\begin{array}{l}1 \text { plus } 1 \\
\text { ventral }\end{array}$ & 51,88 & 5 & 139 & 180 & .77 & none \\
\hline $7 / 22 / 02 \mathrm{E}$ & 1 & 1 & 27 & 28 & 162 & 193 & .83 & cell body \\
\hline 6/29/02 & 1 & 1 & 10 & 44 & 79 & 154 & .51 & none \\
\hline $6 / 22 / 02 \mathrm{~A}$ & 1 & 1 & 79 & 16 & 156 & 119 & 1.3 & none \\
\hline $6 / 22 / 02$ B & 1 & $\begin{array}{c}2 \text { plus } 1 \\
\text { dorsal }\end{array}$ & 14 & 12,22 & 162 & 82 & 1.9 & cell body \\
\hline $6 / 24 / 02$ & 1 & 1 & 67 & 26 & 67 & 26 & 2.5 & cell body \\
\hline $6 / 29 / 02$ B & 1 & 2 & 17 & 36,64 & 98 & 100 & .98 & cell body \\
\hline $6 / 29 / 02 \mathrm{C}$ & 1 & 1 & 45 & 10 & 45 & 53 & .84 & cell body \\
\hline $6 / 29 / 02$ D & 1 & 1 & 64 & 5 & 120 & 209 & .57 & cell body \\
\hline $\begin{array}{c}\text { Russell and Moore, } \\
1999 \mathrm{~A}\end{array}$ & 2 & 1 & 27,26 & 3 & 88 & 58 & 1.5 & cell body \\
\hline $\begin{array}{c}\text { Russell and Moore, } \\
1999 \mathrm{~B}\end{array}$ & 1 & 1 & 44 & 6 & 44 & 139 & .31 & cell body \\
\hline Smith A & 1 & 1 & 15 & 6 & 650 & 601 & 1.08 & $\begin{array}{c}\text { lateral } \\
\text { dendrite }\end{array}$ \\
\hline Smith B & 1 & 1 & 192 & 137 & 789 & 192 & 4.1 & $\begin{array}{c}\text { lateral } \\
\text { dendrite }\end{array}$ \\
\hline Smith C & 1 & 1 & 74 & 151 & 141 & 151 & .93 & $\begin{array}{c}\text { lateral } \\
\text { dendrite }\end{array}$ \\
\hline Smith D & 1 & 1 & 98 & 161 & 574 & 302 & 1.6 & $\begin{array}{c}\text { lateral } \\
\text { dendrite }\end{array}$ \\
\hline Smith $\mathrm{E}$ & 2 & 1 & 174,222 & 248 & 396 & 248 & 1.5 & $\begin{array}{l}\text { lateral } \\
\text { dendrite }\end{array}$ \\
\hline
\end{tabular}

Table 1. Dendritic Morphology of MSO cells. Shaded values from Russell and Moore, 1999 and from Smith, 1993. From our labeled neurons, 10 out of 29 neurons had more 
than one primary dendrite per side, whereas the remainder had only one dendrite per side. The cells from our study and from Russell and Moore, 1999 come from gerbils and the cells from Smith, 1993 come from guinea pigs. 


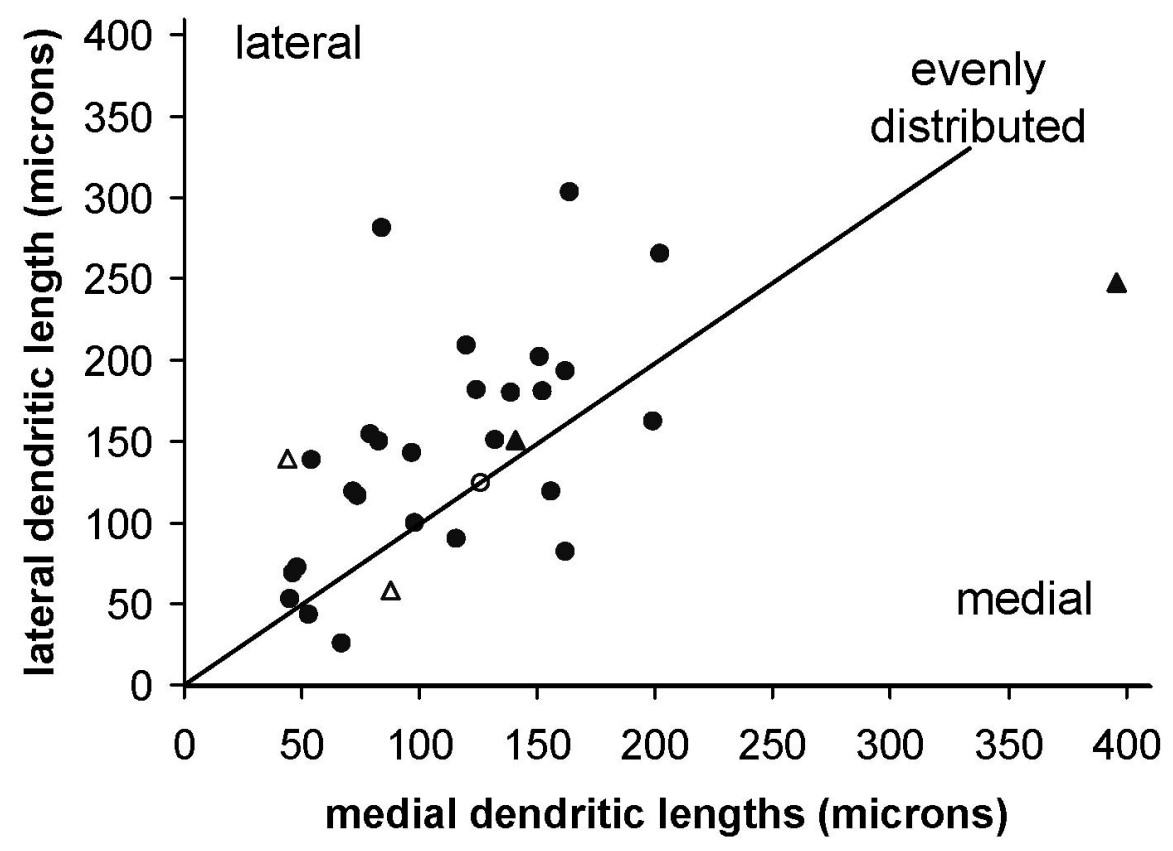

Figure 2. Scatterplot of summed medial versus lateral lengths of thick dendrites.

The lengths of only thick dendrites of MSO cells were measured and the medially oriented dendrites were compared to laterally oriented dendrites. Data points above the diagonal line represent a bias in summed dendritic lengths towards the lateral side. Data points falling directly on the line had evenly weighted dendritic fields. The cell used to create the model is plotted as an open circle and falls directly on the line. Cells from Russell and Moore, 1999 and Smith, 1993 are plotted as open and filled triangles, respectively. 


\begin{tabular}{lc}
\hline Model Parameter & Value \\
\hline temperature & $30^{\circ} \mathrm{C}$ \\
axial resistance & $160 \mathrm{M} \Omega / \mathrm{cm}$ \\
membrane capacitance & $1 \mu \mathrm{F} / \mathrm{cm}^{2}$ \\
dt (integration interval) & $0.1 \mathrm{msecs}^{*}$ \\
leakage conductance & $.001 \mathrm{mho} / \mathrm{cm}^{2}$ \\
number of inputs & $1-6$ \\
$\begin{array}{l}\left(\alpha_{\text {ex }}\right) \text { time to peak of alpha } \\
\text { function } \\
\text { excitatory synapse reversal } \\
\text { potential }\end{array}$ & $0.1-2(\mathrm{~ms})$ \\
\end{tabular}

Table 2. List of parameters used in the model MSO cell. * The value of $\mathrm{dt}$ was selected to be identical to the sampling frequency during electrophysiological experiments $(10 \mathrm{kHz})$. 


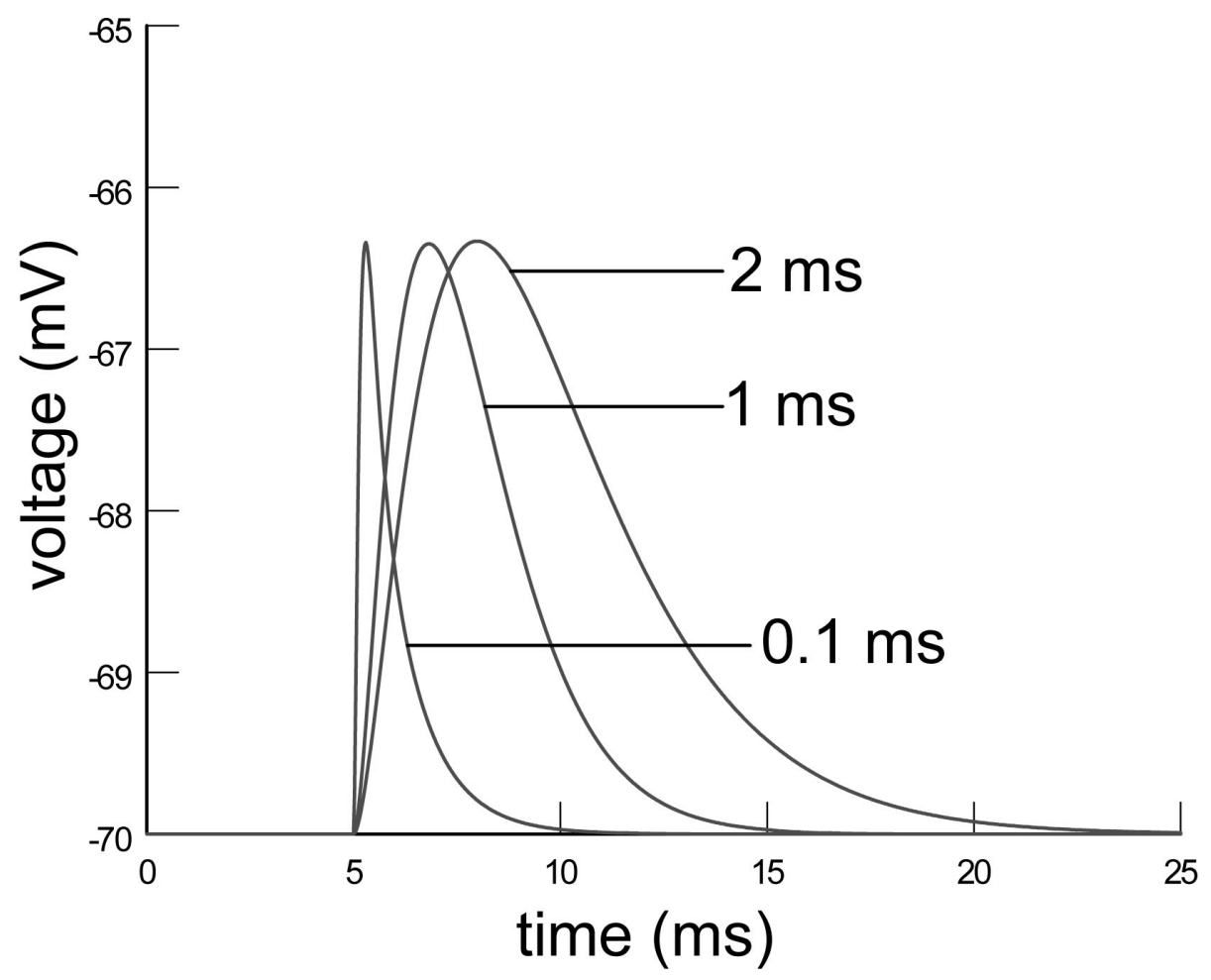

Figure 3. EPSPS recorded from the soma of the model cell.

Decreasing $\alpha$ (the value of $\alpha$ is listed beside each trace) decreased the time to peak and duration of the EPSP. 


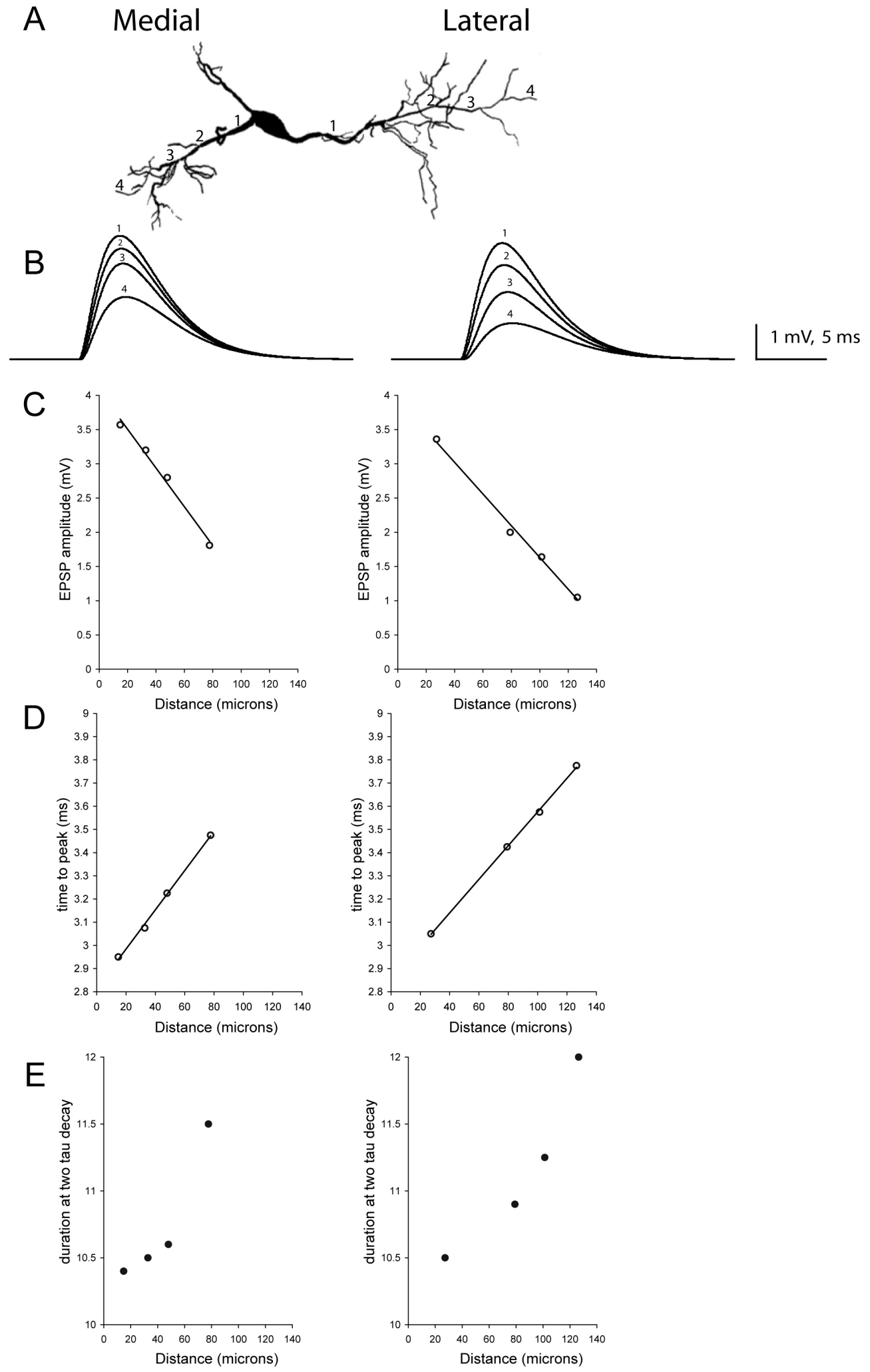

Figure 4. The amplitude, time to peak and duration of EPSPs change with synaptic location. 
A. Synapses, numbered 1-3, were placed on thick dendrites, whereas number 4 was placed on a thin dendrite. The placements of synaptic inputs are indicted by numbers. B. EPSPs recorded from the soma from each of the synaptic locations are indicated by numbers. C. The peak amplitude of the EPSP declined with increasing distance from the somal recording site. D. The time to peak of the EPSP was delayed as the synaptic inputs were placed more distally. E. The duration of the EPSP was measured as the time taken to decay two time constants from the peak of the EPSP. As the synaptic inputs were placed more distally, the total duration of the EPSP increased. 

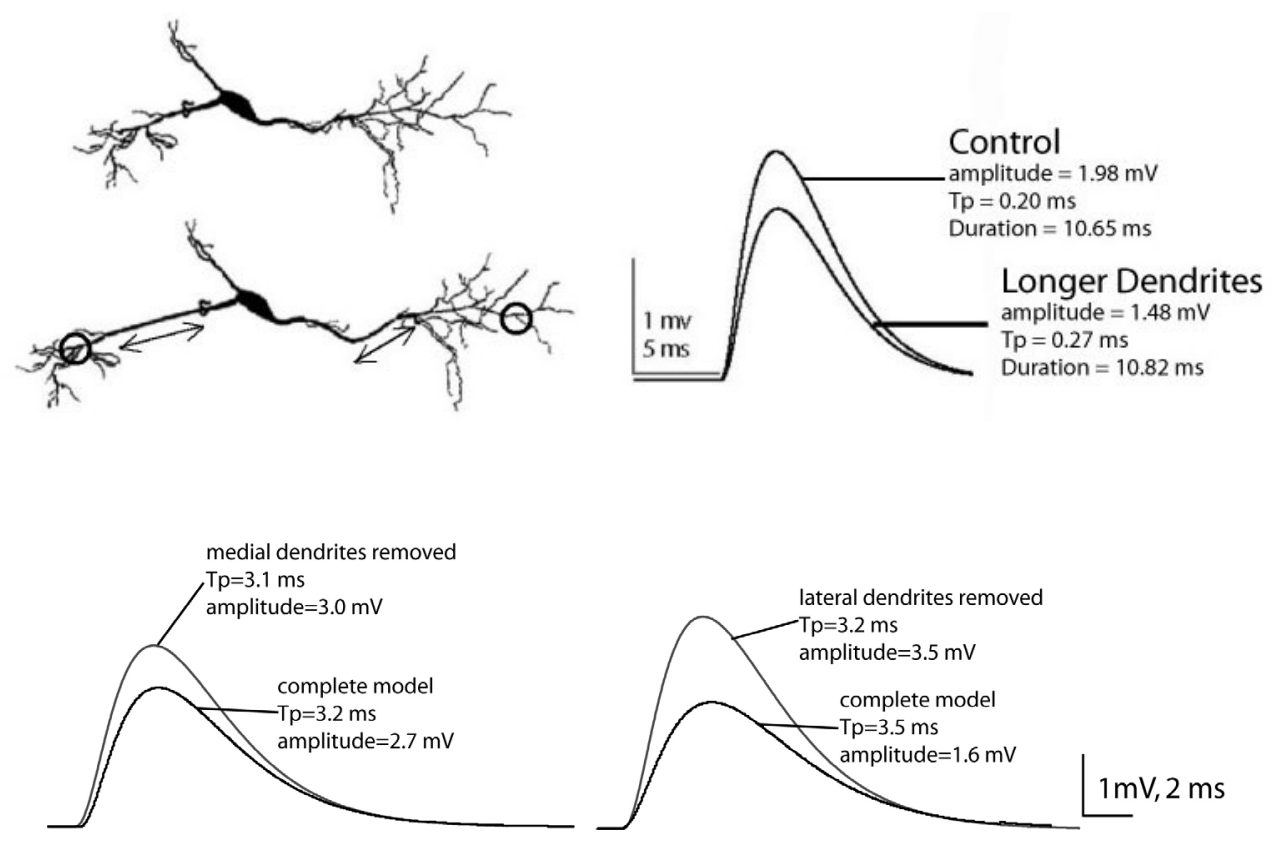

Figure 5. Effects of structural variations of the model cell on EPSPs recorded in the soma.

Top: To test the effects of dendritic length on the EPSP, we increased the lengths of two primary dendrites of the model MSO cell and recorded EPSPs in the soma. We injected excitatory current injections at the positions indicated by circles (position 3 in Figure 4A). Top right: The amplitude of EPSPs in the cell with the longer dendrites was attenuated by $0.5 \mathrm{mV}$. Bottom: We further tested the model by removing either the medial or lateral dendrites and found that the amplitudes of EPSPs of the cell with dendrites removed were increased. 

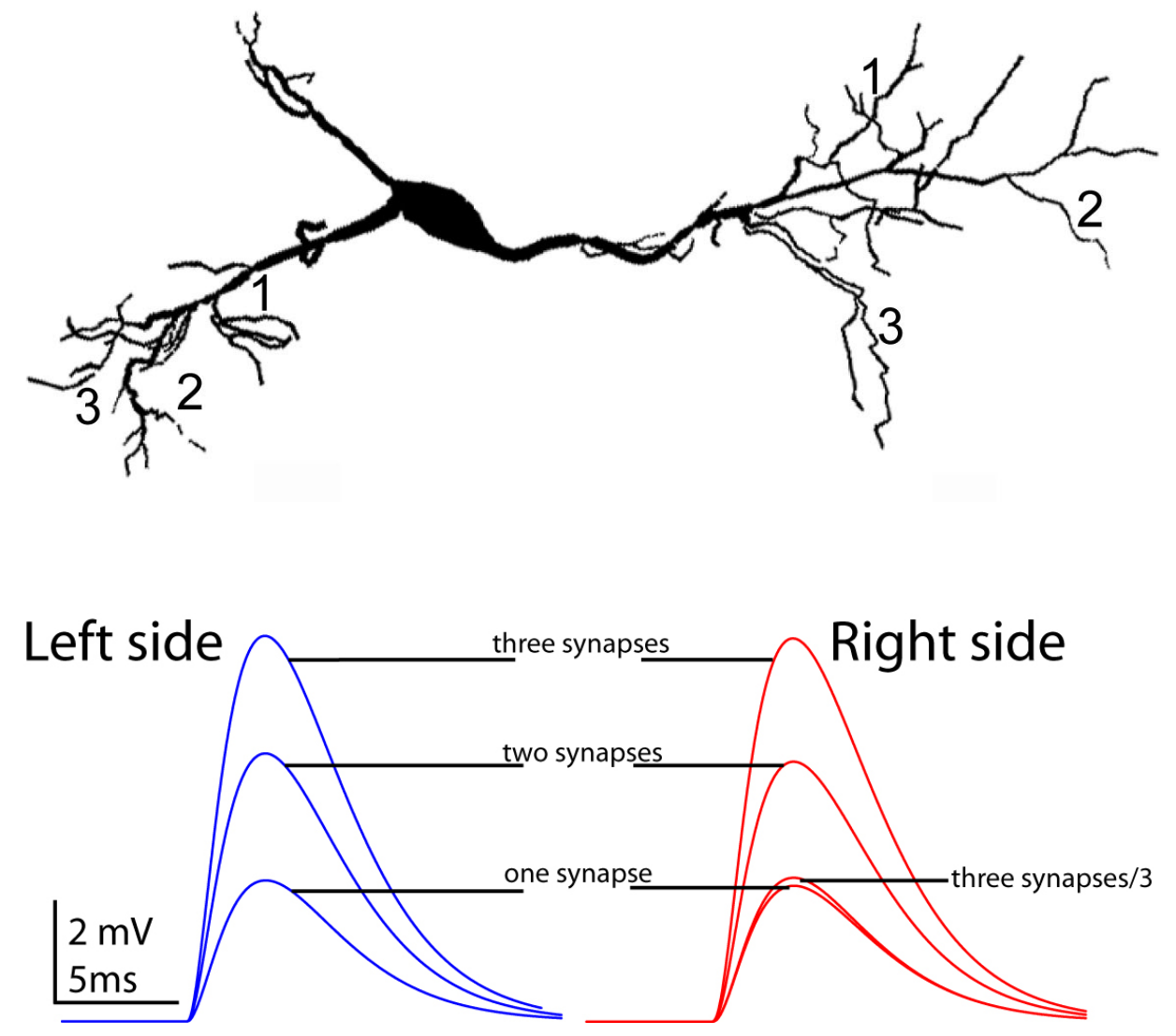

Figure 6. Addition of excitatory synapses increases the amplitude of the voltage response in the soma.

Top: Three synapses (excitatory conductance $0.001 \mu \mathrm{S}$ ) were added one at a time to each side of the passive model MSO cell. The numbers next to each dendrite indicates the placements of synaptic inputs. Bottom: Voltage recordings from the soma of the model cell. Addition of the synapses increased the amplitude of the EPSP but did not change the time to peak or the overall duration of the voltage response. Dividing the conductance of each of the synapses by the total number of synapses per side reduces the amplitude to nearly the same response as one synapse acting alone. (Marked as three synapses/3 on right side and is superimposed on the lowest trace on the left side. 


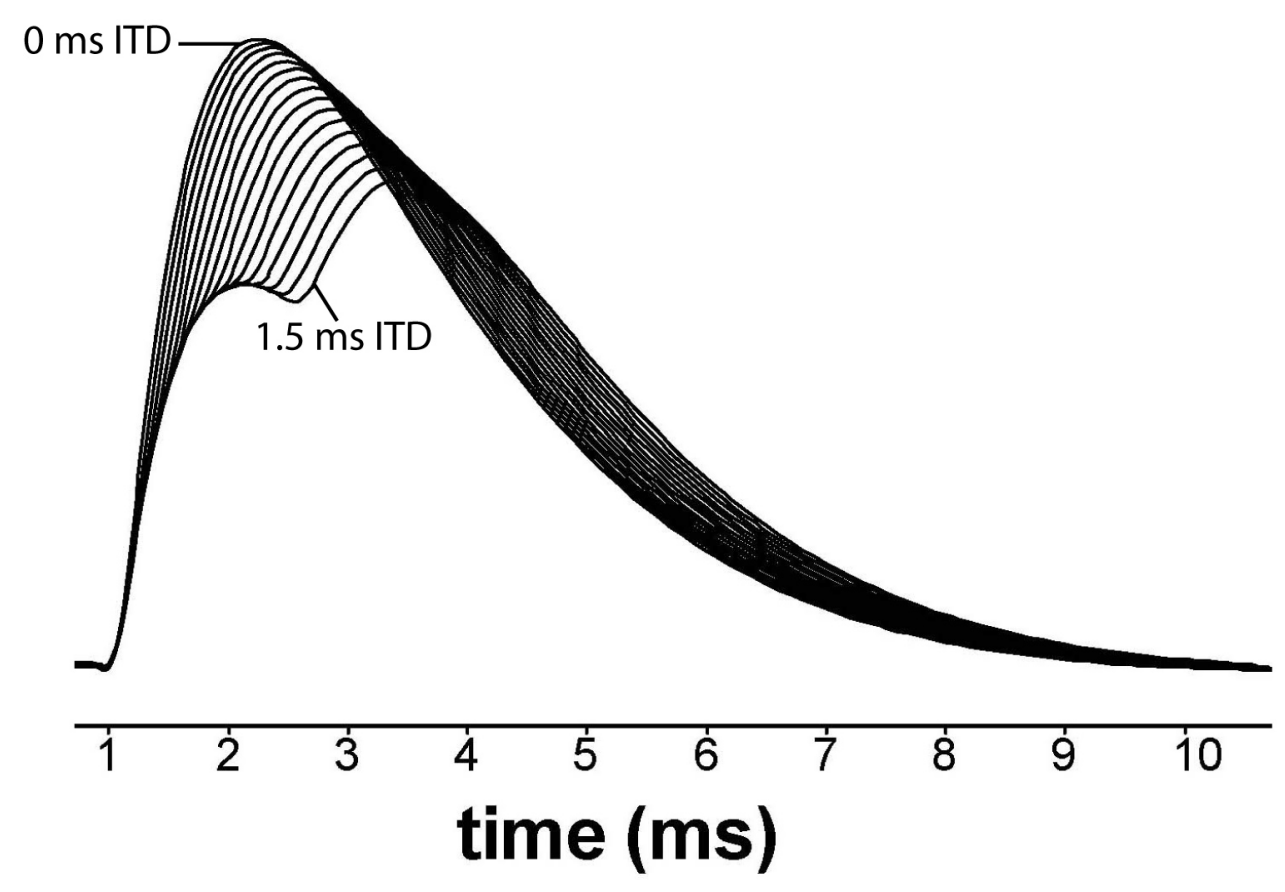

Figure 7. EPSPs recorded from the model MSO cell at different simulated ITDs. We activated one dendritic field of the model cell relative to the other in increments of $100 \mu \mathrm{s}$ in order to simulate changes in ITD. Increasing the ITD caused a biphasic response and decreased the maximum amplitude attained. 
Chapter 5: The roles of voltage gated potassium channels and synaptic inhibition on the coding of ITDs by medial superior olive cells. 


\section{Introduction}

Neurons integrate a delicate balance of excitatory and inhibitory inputs over a background population of ionic conductances. However, the interactions of synaptic inputs with intrinsic cellular membrane properties are typically studied in isolation and are therefore difficult to relate to a neuron's appropriate functional context. We examined the contribution of the cellular properties of neurons of the medial superior olive (MSO) to their identified role in sound localization. MSO cells alter their spike activity based upon microsecond differences in the arrival time of sound at the two ears (interaural temporal delay or ITD), yielding differences in arrival times of bilateral excitatory and inhibitory synaptic inputs. These neurons are defined by specialized mechanisms to detect small changes in ITD, which have been attributed to a population of potassium conductances activated by depolarization and to precise timing of inhibitory inputs. We devised an experimental approach to assay the role of potassium conductances in temporal integration of excitatory inputs independent of, and then in combination with, inhibitory neurotransmission. Our first goal was to determine the shape of the coincidence detection window to excitatory inputs only. This information has not been measured in vivo due to the difficulty of obtaining intracellular recordings from these cells, variance in the timing of each monaural input from cycle to cycle of a periodic stimulus, and the inability to completely antagonize inhibitory neurotransmission. To obtain this information, we employed an in vitro brain slice preparation to inject composite "synaptic" EPSP waveforms of different simulated ITDs into MSO neurons, with or without pharmacological blockade of the Kv1 family of potassium channels.

We proposed to study the relative timing and integration of EPSPs with IPSPs in living MSO cells. Our hypothesis was that delayed inhibition would shorten EPSPs and decrease the coincidence window in which two EPSPs could sum to generate action potentials. The time of arrival of IPSPs compared to excitation in the MSO is unknown, but given the physiological properties of velocity along GBC axons and extremely short synaptic delays, IPSPs could precede excitation. A recent modeling study of MSO cells showed that inhibitory 
inputs that precede excitation in a model MSO cells plays a major role in the shapes of ITD curves (Brand et al. 2002). Therefore we studied the effects of temporally varying the onset of inhibition relative to excitatory inputs in living MSO cells (both preceding and following) and found that the timing of inhibition can drastically affect the responses to current injection stimuli and are a major contributor to the shaping of ITD curves. 


\section{Methods}

\section{Electrophysiology}

The preparation of brain slices for electrophysiology is described in the Methods of Chapter 3.

\section{Pharmacology}

To determine the effect of potassium channels on MSO cell firing properties and ITD coding, blockers of voltage-gated potassium channels were added to the bath ( $\alpha$-dendrotoxin 10,50 and $100 \mathrm{nM}$ or 4-aminopyridine $1 \mathrm{mM}$ ). The concentrations of dendrotoxin used have been shown to effectively and selectively block Kv 1.1, Kv 1.2 and Kv 1.6 potassium channels (Harvey and Karlsson 1980; Harvey 2001).

To study the effects of inhibitory inputs alone, antagonists to glutamatergic excitatory neurotransmission (CNQX to block AMPA receptors, APV and MK801 to block NMDA receptors; $10 \mu \mathrm{M}$ each) were added to the bath during these experiments to block activation of the excitatory inputs originating from spherical bushy cells (SBCs) of the $\mathrm{CN}$.

\section{Simulated binaural excitation}

In order to control for uneven sectioning of excitatory afferents from each cochlear nucleus during slice preparation (Smith et al. 1998), and to have better control of excitatory stimulation, we injected simulated binaural composite EPSP waveforms through the recording pipette into the soma of living MSO neurons (Figure 1). Each simulated ITD waveform were injected 10 times at a stimulus frequency of $100 \mathrm{~Hz}$. We tested 16 ITDs, starting with zero ITD, in increments of $100 \mu \mathrm{s}$, to a maximum of $1.5 \mathrm{~ms}$. Each set of 10 stimuli at one ITD was followed by a rest period of 500 msecs, and was repeated three times prior to injecting the next ITD. Example current injections at 0, 0.5, 1.0 and $1.5 \mathrm{~ms}$ ITD are shown in

Figure 1. A range of scaling factors for the current injections was injected into each cell to investigate the role of signal amplitude on MSO cell spike generation. 
We generated plots of normalized spike counts versus simulated ITD for responses from the current injections. For example, a normalized value of 1.0 corresponds to an ITD where action potentials (spikes) occurred on all 10 cycles of the ITD (entrainment) being tested during all three repetitions. For phasic cells, slopes from ITD curves were measured from the longest ITD that gave entrainment (normalized spike count of 1.0) to the shortest ITD that did not yield spikes (normalized spike count of 0.0 ; called 100 to 0 slopes). All slopes are reported as the decrease in normalized spike counts per $100 \mu \mathrm{secs}$. The longest ITD that resulted in a spike count of 1.0 we termed the "corner ITD". For example the fitted line in the inset of Figure 7 is fit from the corner ITD to the ITD that elicited zero spikes. Tonic cells did not have normalized spike counts that fell to 0.0 , so the minimum of the ITD curve was used instead of the zero rate ITDs. In some instances, where indicated, the slopes of the steepest portion of the ITD curve (between 0.8 and 0.2 spike counts; called 80 to 20 slopes) were measured.

\section{Inhibition}

To determine baseline parameters for inhibitory synaptic inputs, we tested the frequency following ability of 20 MSO cells in their generation of IPSPs. IPSPs were generated to brief shocks of the MNTB (+/- 1-100V; $200 \mu \mathrm{sec}$ duration). Test frequencies of $10,50,75,100,125,150$, and $200 \mathrm{~Hz}$ stimulation of the MNTB were used, in the absence of any excitatory current injection. As a measure of the cell's frequency following ability, we counted the number of IPSPs elicited in the MSO and divided by the number of shocks delivered to the MNTB to yield normalized IPSP counts at each stimulation.

We next determined if $100 \mathrm{~Hz}$ stimulation frequency of the MNTB resulted in synaptic depression of IPSPs, i.e. a sequential decrease in the amplitude of IPSPs with increasing stimulus number. We made measurements of IPSP amplitudes (Clampfit, Axon Instruments) from 13 cells in response to shocks of the MNTB and plotted the measured amplitudes against their number in the train. 


\section{Simulated binaural excitation coupled with activation of inhibitory inputs}

After determining the action potential response of simulated excitation alone, we sought to determine the influence of synaptic inhibition on ITD coding. We varied the temporal relationship between excitation and inhibition by shocking the MNTB ( $\pm 100 \mathrm{~V}, 200 \mu$ s duration) at systematic test points during the simulated excitatory current injection. The stimulus voltage was adjusted to the minimum necessary to entrain a response at $100 \mathrm{~Hz}$. The same excitatory current injection stimuli to simulate ITDs as described above were used. We tested inhibition that arrived prior to (leading), synchronous with, and following (lagging) the start of simulated excitatory current injections (Figure 2). Shocks were delivered to the MNTB over a range of times relative to the onset of EPSP waveform injection from $5 \mathrm{~ms}$ leading to $2.0 \mathrm{~ms}$ lagging in $0.5 \mathrm{~ms}$ increments. The MNTB was activated at a frequency of $100 \mathrm{~Hz}$, identical to that of the simulated excitatory current injection, so that each cycle of the excitatory current injection had an associated inhibitory input. Plots of ITD curves without activation of the MNTB (control) were compared to curves with inhibition. 


\section{Results}

\section{Potassium channels determine the phasic response}

As reported in Chapter 3, phasic neurons constituted nearly $4 / 5$ of our sample after PD 11 (Figure 3, left). In addition to generating 1-3 action potentials at stimulus onset (termed the phasic response), responses to current injection included a hyperpolarization-activated membrane potential change and a nonlinear relationship between injected current and voltage (I-V curve in inset in Figure 3 far right). The slopes of I-V curves for values below resting membrane potential were on average lower than those above the resting membrane potential $($ depolarized resistance $=47.3+/-10 \mathrm{M} \Omega$; hyperpolarized resistance $=$ $61.6+/-15 \mathrm{M} \Omega ; p<0.001$ ). The hyperpolarization-activated membrane potential change forced the voltage back towards the resting membrane potential that resulted in a voltage "sag" (stars indicate peak voltage response just prior to sag in Figure 3). Tonic neurons, in contrast, generated sustained spike activity when depolarized, but still exhibited a voltage sag (Figure 3, tonic). Slopes of I-V curves, an indicator of input resistance, of tonic neurons were steeper at hyperpolarized voltages than those of phasic neurons (tonic: $208+/-65 \mathrm{M} \Omega$; phasic: $61.6+/-15 \mathrm{M} \Omega ; p<0.001$ ).

We assayed the roles of $\mathrm{Kv} 1$ family potassium channels in the transient response of $27 \mathrm{MSO}$ neurons through the application of the selective potassium channel blocker $\alpha$-dendrotoxin ( $\alpha$-DTX). Under control conditions (no drug), the I-V curve was non-linear and had steeper slopes at more hyperpolarized voltages compared to depolarized voltages (Figure 3 arrow in I-V curve to the right). Application of $\alpha$-DTX, which blocks Kv1.1, 1.2 and 1.6 alpha subunits at the concentrations we used $(10,50$ and $100 \mathrm{nM})$, to MSO neurons converted the phasic response into a tonic pattern of action potentials in all 27 cells, but did not eliminate the hyperpolarization-activated current (star in Figure 3). We were not able to discern any changes in input resistance for depolarizing current injections because after the application of $\alpha$-DTX very slight depolarization from rest caused the cell to reach threshold and fire repetitive action potentials, thus 
preventing measurement of steady state voltage responses. The change in threshold was most likely due to an increase in input resistance but was also in part due to a very small depolarization in the resting membrane potential $(+2.3$ $m V)$. Application of $\alpha$-DTX caused greater shifts in the voltage for hyperpolarizing current injections compared to control situations. These shifts in voltage caused the resultant I-V plots to become steeper (indicating a higher input resistance) at more hyperpolarized voltages. When we measured the input resistance prior to and following the application of $\alpha$-DTX to bath, we found a significant increase in input resistance at hyperpolarized voltages (control: $40.7+/-10.4$; $\alpha$-DTX: 53+/$13.1 ; p<0.05 ; \mathrm{n}=27)$.

\section{Three types of afterhyperpolarizations follow the action potential in MSO neurons}

We observed distinct differences between phasic and tonic neurons in the afterhyperpolarizations (AHP) that followed action potentials. In all of the phasic neurons studied, action potentials were followed by fast (1-2 ms) AHPs, which were similar in appearance in all cells studied (Figure 3B). Application of $\alpha$-DTX to phasic neurons did not reveal AHPs with multiple components. The AHPs of phasic neurons were of similar magnitude but the duration of the AHPs was increased (Figure 3E).

Tonic neurons had two distinguishable AHPs. In 6 out of 26 tonic neurons studied, action potentials were followed by AHPs that had three distinct components. First a fast afterhyperpolarization (fAHP), followed by an intermediate afterdepolarization (iADP) and finally by a slow afterhyperpolarization (sAHP; Figure 3B and C). The peak of the iADP occurred on average $1.2 \pm 0.3 \mathrm{~ms}$ following the maximum hyperpolarization of the fAHP in these six cells. The maximum hyperpolarization of the sAHP occurred on average $11.0 \pm 3.9 \mathrm{~ms}$ following the peak of the iADP.

In the remaining 20 tonic neurons, no distinguishable iADP was observed. These neurons displayed a hyperpolarization followed by a slow rising (tens of milliseconds) repolarization, which lead into another action potential. The action potential response was graded with stimulus intensity, i.e. the stronger the 
current, the higher the rate of firing.

\section{Phasic and Tonic cells differ in sensitivity to ITD}

Next, we investigated the sensitivity of MSO neurons to simulated ITD stimuli. Example voltage responses from living MSO cells in response to control (no stimulation of inhibitory inputs) simulated ITDs of $0,0.5$ and $1.0 \mathrm{~ms}$ are shown in Figure 4 (top panel). We plotted ITD versus normalized spike counts for several amplitudes of simulated ITDs (labeled as "gain" in the figure). The phasic neuron displayed in the graph exhibited a steep decline in firing rate from entrainment to the stimulus to zero activity, over an ITD range of $500 \mu \mathrm{sec}$ (Figure 4). In this example, the decline in spikes counts was -0.2 spikes counts per $100 \mu$ secs ITD, a value representative of a cell with a slightly faster than average decline (average $=-0.14+/-0.07 ; \max =-0.35 ; \min =-0.09$; further examples and detailed description below). In contrast, tonic neurons exhibited very little sensitivity to ITDs (Figure 4, right panel) over the entire range of stimulus amplitudes that were applied to the cell (average $=-0.01+/-.01 ; \max =-$ 0.08; $\min =0$ ). These slopes were measured from the corner ITD to the minimum spike count value for phasic cells. Tonic slopes (since most did not entrain to the stimuli) were measured from the maximum spike count to the minimum spike count. These slopes are referred to 100 to 0 slopes.

To determine if the gain of the injected current stimulus affected the slope of the ITD curve, we measured the slopes of 128 ITD curves, from between 0.8 to 0.2 normalized spike counts (also referred to as 80 to 20 slope) from 43 phasic neurons. The 80 to 20 slope was chosen because it represents the steepest portion of the curve and the most physiologically relevant segment of the ITD plot (see Discussion). When the 80 to 20 slope was unable to be calculated, the steepest part of the curve (as determined by eye) was recorded. We separated the ITD curves into five categories depending upon the corner ITD that the injected current stimulus elicited. All of the 128 ITD curves that we generated and their corresponding 80 to 20 slopes are shown in Figure 5 (A-E; five separate pages).

The first category was defined as stimulus gains that did not elicit 
entrainment to all cycles of the stimulus at zero ITD ("below" 1.0 on the ordinate; Figure 5A). The histogram of "below" ITD curves were mostly clustered towards higher values (inset in Figure 5A), indicating shallow ITD curves (mean slope = $0.15+/-0.1$ spike counts per $100 \mu$ secs ITD), but do include a few moderately steeper slope values (range: -.01 to -0.36 spike counts per $100 \mu$ secs; $n=40$ ITD curves).

The remaining ITD curves were separated into four categories based upon their corner ITD ( 0 to $0.3,0.4$ to $0.7,0.8$ to 1.1 and 1.2 to $1.5 \mathrm{~ms}$ ). The 0 to 0.3 ms corner ITD category had on average steeper slope values than the "below" category (mean $=-0.20+/-0.11 ; n=29$ ) with most slopes valued at -0.25 (range: -0.05 to -0.53 ; Figure $5 \mathrm{~B}$ ).

The 0.4 to $0.7 \mathrm{~ms}$ corner ITD category had a similar mean slope value to the 0 to $0.3 \mathrm{~ms}$ category (mean $=-0.20+/-0.11 ; \mathrm{n}=28$ ) but a slightly higher modal value (-0.31; range: -0.2 to -0.43 ; Figure $5 \mathrm{C}$ ).

The 0.8 to $1.1 \mathrm{~ms}$ corner ITD category had a higher mean slope value than the 0 to 0.3 and 0.4 to 0.7 categories (mean $=-0.26+/-0.13 ; n=28$ ) and most slopes were binned at -0.25 (range: -0.1 to -0.63 ; Figure $5 \mathrm{D}$ ).

We only sampled a small number of ITD curves in the 1.2 to $1.5 \mathrm{~ms}$ corner ITD category $(n=3)$. None of the curves decreased to spike counts below 0.6 and we therefore measured the steepest portion of the curve. The measured values of this category were $-0.16,-0.2$ and -0.3 (mean $=-0.22+/-0.07$; Figure $5 E)$.

We did not find a statistically significant difference in the slope values between the four categories (all except the "below" category) that elicited entrainment (ANOVA between group comparison: $p=0.22$ ). When those four categories were grouped together $(n=88$; average slope $=-0.22+/-0.12)$ were compared to the slope values of the "below" category $(n=40$; average slope $=$ $0.15+/-.1$ ), we found that the "below" category had slopes that were significantly more shallow than the group that elicited entrainment $(p=0.001)$.

The identical simulated ITD protocols were performed on the tonic set of neurons. We generated 19 ITD curves from 13 neurons. Most curves were 
strikingly shallow (Figure 6) and two neurons exhibited no sensitivity to the simulated ITD stimuli. The average slope of the ITD curves for tonic cells were $0.01+/-.01(\max =-0.08 ; \min =0)$ and most slope values were -0.1 (histogram in Figure 6). A comparison of the ITD curves from tonic cells to those of phasic cells indicated that phasic cells had significantly steeper slopes $(p<0.0001)$. When the two tonic neurons that exhibited no sensitivity were excluded from the analysis the statistical significance remained. When the ITD curves from tonic neurons were compared to the "below" category of phasic cells we found that phasic neurons had significantly steeper slope values $(p<0.0001)$.

To determine if differences in ITD sensitivity are related to the input resistance of the cell, we plotted input resistance versus slope of the ITD curve (Figure 7; "below" values excluded for phasic cells). To make direct comparisons, 100 to 0 slopes were calculated for phasic population. A clear distinction between phasic and tonic cells was found. Phasic cells had low input resistances (less than $60 \mathrm{M} \Omega$ ) and steep ITD curves, whereas all of the tonic cells had high input resistances (between 60 and $240 \mathrm{M} \Omega$ ) and shallow ITD curves. When phasic cells were examined alone we did not find a strong correlation between the slope of the ITD curve and input resistance, decreasing an average .1 normalized spike counts per $18.1 \mathrm{M} \Omega$ increase in resistance.

\section{Blockade of $\alpha$-DTX sensitive potassium channels decreased the cell's sensitivity to ITDs}

We tested whether blocking $\alpha$-DTX sensitive potassium channels decreased the sensitivity of MSO neurons to simulated ITDs by superfusing 10 , 50 or $100 \mathrm{nM} \alpha$-DTX into the recording chamber. We found that application of $\alpha-$ DTX to the bath drastically decreased sensitivity to changes in ITD in phasic cells (Figure 8 left; $n=19$ cells; 15 cells at $100 \mathrm{nM}, 2$ at 50 and 2 at $10 \mathrm{nM} \alpha$-DTX). As indicated above, blocking the $\alpha$-DTX sensitive potassium channels increased the input resistance of the cell (control: $40.7+/-10.4$; $\alpha$-DTX: $53+/-13.1$ ) and the current required to generate action potentials was decreased, so the cells entrained to simulated ITD stimuli. We tested whether decreasing the gain of the excitatory current injection to amplitudes below that yielding entrainment would 
restore any sensitivity to ITDs with $\alpha-D T X$ in the bath. For example, in the cell shown in Figure 8, we decreased the gain of the current injection to $70 \%$ of the control value. With $100 \mathrm{nM} \alpha$-DTX in the bath, the slope of the ITD curve remained very shallow and the response showed only slight sensitivity to ITDs. Lower gains compared to control values were tested on all cells and decreasing the gain did not restore sensitivity to simulated ITDs ( $n=19$ cells) to control values.

We measured the slopes of ITD curves both before and following application of $\alpha$-DTX to the bath for these 19 cells. There was a reduction in the slope of the ITD curves for all applications of $\alpha$-DTX and the magnitude of the effect was independent of concentration. Addition of $\alpha$-DTX (all concentrations) into the bath decreased the slope of the ITD curve from $-0.17+/-.08$ (control) to $-0.02+/-0.02(\alpha-D T X)$. Addition of $50 \mathrm{nM} \alpha$-DTX caused an average decrease in slope of the ITD curve from $-0.14+/-0.07$ (control) to 0 ( $\alpha$-DTX; $n=2$ ). Application of $10 \mathrm{~nm} \alpha$-DTX caused a decline in slope from $-0.1+/-0.1$ to -0.003 $+/-0.004$ (a $88 \%$ reduction in slope; $n=2$ ).

The limited ITD sensitivity associated with tonic cells was nearly abolished following $\alpha$-DTX application ( $n=2$; Figure 8 ). The slope of the ITD curve for tonic cells decreased from $-0.045+/-0.02$ (control) to $-0.02+/-0.02(\alpha-D T X ; n=$ 2).

\section{Inhibitory post-synaptic potentials}

We sought to determine the role of inhibitory inputs in the coding of ITDs by MSO neurons. As a first step, we recorded IPSPs from MSO cells elicited from different frequencies of stimulation of the MNTB (10-200 Hz; see Methods). Only animals older than PD 11 were used based upon our findings that in older animals, IPSPs become faster and their durations are less variable (see Chapter 3). After PD 11 the average IPSP duration was $7 \pm 1 \mathrm{~ms}$. We also made measurements of the time constant of recovery of IPSPs and found that after PD 11 the average IPSP time constant was $3.0 \pm 2 \mathrm{~ms}$. In our current study of inhibition, only 2 cells out of 20 could generate IPSPs up to $200 \mathrm{~Hz}$, whereas 11 
out of 20 cells could generate IPSPs at a $100 \mathrm{~Hz}$ stimulation frequency. The remaining 9 cells responded on at least $90 \%$ of the trials at a $100 \mathrm{~Hz}$ stimulation frequency. On average, MSO cells could respond to stimulation of the MNTB with IPSPs on $97 \%$ of the trials. An example voltage recording of IPSPs in an MSO cell that entrained to MNTB stimulation is shown in Figure 9A. The response to stimulation of the MNTB decreased with increasing frequency of stimulation, to an average value of $65 \%$ at $200 \mathrm{~Hz}$ stimulus frequency (Figure 9B).

Synaptic depression might play a role in a cell's sensitivity to ITDs and confound our trial by trial investigation of inhibitory interactions with simulated EPSPs. We therefore quantified the amount of synaptic depression by measuring the amplitudes of IPSPs from a series of 14 stimuli at $100 \mathrm{~Hz}$ from 13 different cells (see Methods). We plotted the normalized amplitude of the first IPSP versus shock number in the frequency train. The amount of synaptic depression was assessed by comparing the amplitudes of early IPSPs to those later in the train, focusing on the first 10 events, because our simulated ITD protocol only used 10 successive stimuli.

The amplitudes of IPSPs were highly variable throughout the stimulus train. In general, the amplitude of the $10^{\text {th }}$ IPSP in the train was less than the first, but could be preceded or followed by an IPSP that was larger in magnitude (Figure 10, top). Therefore, we classified these IPSPs as not exhibiting synaptic depression. In three cells, however, IPSP amplitudes declined steadily during the stimulus train (Figure 10, bottom), although the amplitude on the $10^{\text {th }}$ stimulus was at least $30 \%$ and as high as $70 \%$ of that on the first stimulus.

\section{Activation of inhibitory inputs during simulated excitation: effects of lagging inhibition}

We studied the potential for inhibitory inputs to shape ITD curves by activating IPSPs, via stimulation of the MNTB, at varying times relative to the start of simulated excitatory inputs (for details see the Methods section; Figures 8 and 9). Our hypothesis was that inhibition activated following the onset of excitatory inputs (lagging) could speed the decay of non-coincident EPSPs and thereby shorten coincidence detection windows. To test this hypothesis, we 
compared ITD curves of MSO cells both with and without (control) activation of inhibitory inputs. Example voltage recordings are shown in Figure 11. The response of MSO cells to lagging inhibition was investigated in 24 cells. For these 24 cells, we applied a total of 46 different gains of excitatory current injection coupled with inhibition. Of these 46 different gains, 44 had at least one time point for which inhibition was activated after the start of the ITD waveform, that shortened the inhibitory corner ITD compared to control corner ITDs. The effect of lagging inhibition on corner ITDs is shown for four different cells in Figure 12 (arrows in Figure 12A point to corner ITDs). We measured the shift in corner ITDs between the control and lagging inhibition situations. We found that a lagging inhibition shifts corner ITDs to shorter ITDs on average by $404 \pm 280 \mu \mathrm{S}$ $(n=46)$.

\section{Simultaneous onset of inhibition and excitation}

When IPSPs arrived at or very near $(<1 \mathrm{~ms})$ to the start of the simulated ITD current injection (simultaneous onset), there were three types of responses (figures 13-14). For 24 of the 46 gains tested, activation of the MNTB decreased the number of action potentials generated at all ITDs. To measure the amount of action potential suppression, we calculated the difference between normalized spike counts at zero ITD for control and subtracted the normalized spike counts at zero ITD after inhibition. Our analyses were focused at 0 ITD because it yielded the maximum rate of response. For example, activation of inhibitory inputs caused a decrease in .98 normalized spike counts for the example ITD curve shown in Figure 14. On average, simultaneous onset of inhibition decreased the number of action potentials at zero ITD by $.41 \pm .22$ normalized spike counts (Figures 13,14). Three types of responses, two of which had reduced rates at 0 ITD were observed. First, for 9 out of these 24 gains, the decrease in normalized spike counts at zero ITD was followed by an increase in spike counts for the first 100 to $500 \mu$ s and then followed by a decline (Figure 14B). These ITD curves, where spike activity was decreased at short ITDs and then increased at longer ITDs, are termed "domed" shaped responses. 
Second, for 11 out of the 46 gains tested, inhibition from the MNTB completely suppressed all or almost all of the action potentials at all ITDs. An example voltage recording demonstrating this response type is shown in Figure 13 and ITD curves are plotted in Figure 14.

Third, for 5 out of the 46 gains tested the normalized spike count at zero ITD did not decrease, but the corner ITD shifted from longer values (control) to shorter corner ITDs (inhibition). Inhibitory inputs shifted the corner ITD an average of $560 \mu \mathrm{s}$. The responses were similar to those observed with lagging inhibition.

\section{Activation of inhibitory inputs during simulated excitation: effects of leading inhibition}

IPSPs that arrived prior to the start of the simulated excitation hyperpolarized the membrane potential. The hyperpolarizations which occurred can be observed in the voltage recordings (stars in Figure 15). We tested leading inhibitory activation up to $5 \mathrm{~ms}$ prior to the start of the current injection.

To simplify the analyses, we placed the responses to the inhibitory test times into two groups based upon the timing of inhibition. After adjusting for the synaptic delay of each cell, there were 57 leading inhibitory test times that fell between -0.5 to -3.0 ms which constituted our first group. For 37 of 57 gains tested, the ITD curve had decreased normalized spike counts at all ITDs. We measured the difference in normalized spike counts at zero ITD between control and leading inhibition conditions, as in the case of simultaneous inhibition. We found that at zero ITD, inhibition caused an average decline of .47 normalized spike counts. Out of these 57 curves, 28 ITD curves had an increase in spike counts after zero ITD and a decline in rate at longer ITDs (Figure 16). These ITD curves, where spike activity was decreased at short ITDs and then increased at longer ITDs, are termed "domed" shaped responses.

Leading inhibition caused a complete suppression of action potentials in

13 out of the 57 ITD curves. Four of the ITD curves shifted ITD corner values by an average of $375 \mu \mathrm{s}$. For three ITD curves, inhibition had no distinguishable response. 
After adjusting for the synaptic delay of each cell, there were 33 leading inhibitory test times that were earlier than $-3.0 \mathrm{~ms}$ and ITD curves were generated for each. These ITD curves constituted our second group of responses in the leading inhibition category. Out of these 33 curves, 17 were indistinguishable from the control curves. Eleven out of the 33 curves had decreased normalized spike counts for all ITDs compared to control, averaging .23 normalized spike counts at zero ITD. In four of the ITD curves, leading inhibition caused a shift in the corner ITD to an average shorter value of $440 \mu$ s.

\section{Summary}

Overall, large leading times for inhibition caused either no effect or modest suppression of neural activity. As the inhibitory activation time moved closer to the start of the excitatory current injection, more action potentials were suppressed and ITD curves were shifted toward lower spike counts. In some cells, however, spike counts at zero ITD are lower than at longer ITDs, resulting in "dome" shaped ITD curves. The suppression of action potentials was greatest when inhibition was activated at or very near to the start of the simulated ITD injection. Some short lagging delays suppressed action potentials and also caused a decrease in the ITD curves toward lower rates. Longer lagging delays caused a shift in the corner ITD to shorter ITD values, thereby shifting the ITD curve to the left. Example responses from two cells demonstrating the aforementioned shifts in ITD are shown in Figure 17. 
Discussion

\section{Summary}

We recorded the voltage responses of PD 12-19 neurons from the principal cell column of the MSO of gerbils using an experimental approach designed to simulate conditions of different sound source positions. We injected current stimuli into living MSO cells, designed to mimic the voltage response in the cell body that would occur at different ITDs. Action potentials (spikes) were recorded in current clamp mode and plots of spike counts versus ITDs were generated. We found that cells which exhibited phasic responses to depolarizing current injection were much more sensitive to changes in ITDs than tonic cells. The phasic cells' sensitivity to ITDs was dramatically decreased by application of voltage gated potassium channel blockers to the bath. We tested the effects of varying the temporal relationship between excitation and inhibition and found that inhibitory inputs could drastically affect the shape of ITD curves. We determined that both potassium channels and inhibitory inputs work in concert with synaptic excitation to determine the activity of MSO neurons.

\section{Response Types}

\section{Phasic}

The phasic response type is attributable to the presence of one or more types of potassium channels. When potassium channels were blocked with the non-specific potassium channel blocker 4-aminopyridine (4-AP), the phasic response was eliminated and the cell generated multiple action potentials $(n=10$; data not shown). Application of 4-AP converted phasic responses to tonic responses in another study of the MSO (Smith 1995), and other neurons of the auditory brainstem (Oertel 1983; Wu and Oertel 1984; Golding et al. 1999; Bal and Oertel 2001; Dodson et al. 2002a; Manis and Marx 1991). Our results indicate that phasic responses are mediated in part by an $\alpha$-DTX sensitive potassium channel conductance. The venom from the green mamba snake, $\alpha$ DTX, blocks members of Kv1 family of potassium channels that contain Kv1.1, 
1.2 and 1.6 alpha subunits. When $\alpha$-DTX was added to the ACSF, phasic responses were eliminated and replaced with the generation of multiple action potentials. Our results support, albeit indirectly, the presence of the Kv1 family of potassium channels in the MSO and are consistent with another study of the MSO (Svirskis and Rinzel, 2003). Future studies could employ the use of more specific blockers such as dendrotoxin $\mathrm{K}$, (known to block Kv1.1) and tityustoxin (known to block Kv1.2) by using the voltage-clamp method to further dissect the pharmacological profile of MSO cells.

The Kv1 family of channels carries a current that is activated at low voltages (slightly more positive than $-70 \mathrm{mV}$ ) and which only slightly inactivates. This "low threshold" potassium current was first identified in isolated bushy cells of the VCN (Manis and Marx 1991), and was further identified in octopus cells (Bal and Oertel 2001; Ferragamo and Oertel 2002), onset cells of the inferior colliculus (Sivaramakrishnan and Oliver 2001) and the cells of the MNTB (Dodson et al. 2002a). The low-threshold potassium current, due to its negative reversal potential, works to decrease the effectiveness of EPSPs by decreasing the input resistance and speeding of the membrane time constant. The potassium current restricts the effects of synaptic depolarization by decreasing the rate of rise, amplitude and the duration of EPSPs. Decreases in the duration of EPSPs shorten the temporal window in which two or more inputs could sum to generate an action potential, thus improving coincidence detection, and sharpening ITD curves. The potassium current also could work to shorten the duration of action potentials so that the cell is "prepared" for further stimulation.

Cells that exhibited phasic responses were highly sensitive to changes of simulated ITD. The sensitivity to temporal changes in synaptic input of phasic cells is most likely a result of the increased conductance to potassium in the depolarized range, which lowers the input resistance of the cell. Using a simple consideration based upon Ohm's law that voltage is equal to current multiplied by the input resistance, cells with very low input resistances do not generate large changes in membrane potential in response to synaptic current. In contrast, cells with high input resistances, such as those cells that displayed tonic responses, 
generate larger changes in voltage given the same magnitude of synaptic current. This can explain the drastic differences seen in sensitivity to ITDs between tonic and phasic neurons. Interestingly we did not however find any apparent correlation between ITD slope and membrane resistance when phasic neurons were considered alone (Figure 7). These results indicate that the membrane resistance is not the only factor contributing to sensitivity to excitatory drive.

The sensitivity to ITDs of phasic cells was significantly decreased when $\alpha$ DTX was added to the bath. $\alpha$-DTX most likely blocked the low-threshold potassium current and increased the input resistance of the cell. Therefore, with stimuli that simulated long ITDs the spike count had declined to zero, but cells bathed in $\alpha$-DTX responded with action potentials. Blocking voltage-gated potassium channels with $\alpha$-DTX effectively decreases the current needed to generate action potentials. Therefore, the current simulating long ITDs, which reached a smaller peak amplitude than short ITD simulated waveforms, could generate a spike. We compensated for this situation by decreasing the amplitude of the excitatory current injection and found that decreasing the gain did not restore the sensitivity to ITDs. There was a clear distinction between the response type of the cell and it's sensitivity to simulated ITDs (Figure 7). Cells with low input resistances (less than $60 \mathrm{M} \Omega$ ) were all phasic and had very steep ITD curves. Tonic cells (discussed below) had high input resistances and shallow ITD curves. Interestingly, when we analyzed phasic cells alone we did not discover a correlation between input resistance and ITD sensitivity. We conclude that a major component of a neuron's sensitivity to afferent activity results from the total input resistance of the cell but does not dictate the exquisite sensitivity seen among phasic cells. Other cellular components such as the inactivation of voltage gated sodium channels could affect the sensitivity of the cell.

Due to the high sensitivity to changes in ITD we conclude that the phasic pool of neurons that we recorded from in vitro represent the ITD sensitive units recorded in vivo (Yin and Chan 1990; Goldberg and Brown 1969; Spitzer and 
Semple 1995; Brand et al. 2002). Further experiments that make intracellular recordings from in vivo in animals could provide the evidence for this claim.

\section{Tonic}

Based upon the pharmacological results, we conclude that tonic responses are due in part to a lack of an $\alpha$-DTX sensitive potassium current of sufficient magnitude to restrict action potential firing upon sustained current injections. When recording from tonic MSO neurons with $\alpha$-DTX added to the bath, there was an increase in the number of action potentials upon depolarizing current injection. Likewise, when $\alpha$-DTX was added to the bath during stimulation with simulated binaural EPSPs, there was a further decrease in the sensitivity to changes in ITDs. These data indicate that tonic neurons contain $\alpha$ DTX sensitive potassium channels, but the overall conductance in the depolarized range is less than that of phasic neurons. The decreased potassium conductance could be the result of morphological differences between potassium channels or a decrease in the density of channels. It is possible however, that the kinetics of the voltage gated sodium channels are different in tonic neurons than in phasic neurons.

Tonic neurons displayed markedly low sensitivity to simulated ITDs. The low sensitivity of tonic neurons could result from their increased input resistance. The physiological relevance of tonic neurons in the coding of ITDs is unclear. Cells that responded with tonic action potentials were identified in studies of the adult guinea pig MSO (Smith 1995). The tonic cells were histologically identified to come from the marginal cell area of the MSO. Marginal cells surround the principal cell column and define the outer boundary of the MSO and have different response properties than principal cells (Smith 1995). We restricted our sample of MSO cells to only those found within the thin stack of cells in the middle of the MSO, which define the principal cell column. Due to the difficulty in recording from cells after PD 19, the proportion of tonic cells found in the cell column in adult animals is unknown. The possibility that tonic neurons identified at PD 11-19 in vitro further increase their density of voltage-gated potassium 
channels with age and transform into phasic neurons is possible. If the tonic responses of MSO neurons are retained into adulthood, it is likely that the single units reported in the literature recorded from the MSO of gerbils that were not sensitive to ITDs reflect the tonic population of neurons (Brand et al. 2002; Spitzer and Semple 1995). Until the difficulty of recording from the auditory brainstem in older animals is overcome, the role of tonic neurons in the coding of ITDs remains unsolved.

Inhibition

Our results indicate that there is substantial inhibitory processing by MSO cells. The inhibitory inputs to the MSO probably originate at least in part from the MNTB and LNTB and were first implicated in decreasing the spike rate of ITD curves below that seen for monaural stimulation alone, in cells from the MSO (Goldberg and Brown 1969; Yin and Chan 1990) and central nucleus of the inferior colliculus (Kuwada and Yin, 1983). We investigated the effects on MSO cell activity of varying the temporal relationship between excitation and inhibition. We report that, based upon the temporal relationship between excitation and inhibition, ITD curves could be greatly affected. Inhibitory inputs were hypothesized to enhance the peak of ITD curves (Yin and Chan 1990). This certainly could be one possibility, because we show that at certain inhibitory delays corner ITDs were shifted from longer ITDs to shorter ITDs. The shifting of corner ITDs was accomplished for both leading and lagging inhibition, but was most prevalent for lagging inhibition. We could also generate peaks in ITD curves by activation of inhibition where in control situations there were none. This is particularly attractive to explain sensitivity to ITDs at high sound intensities.

Recently, the results of in vivo studies by Brand and colleagues (Brand et al. 2002) suggest that when inhibitory inputs are removed by pharmacological block with strychnine, the peak of the ITD curve is shifted from outside the physiological range to zero ITD. In that study the pharmacology was performed on only five cells and a post-mortem histological verification of the recording site 
was not provided. That the peak ITD naturally occurs outside the physiological range was hypothesized to shift the steepest portion of the ITD curve to midline ITDs where spatial resolution is greatest. Despite its shortcomings, the Brand et al., paper describes an interesting possibility for the role of inhibition. One assumption of the Brand model is that excitatory inputs are presumed to arrive in coincidence at zero ITD and the MSO operates at approximately $50 \%$ of its maximum spike rate. When the sound source moves, however, the spike rate in some cells increases, despite receiving non-coincident input. These responses were explained by using a computer model, which demonstrated that if inhibition arrives prior to excitation ( $0.2 \mathrm{~ms}$ leading in the Brand et al. model) the peak of the ITD shifts away from zero ITD.

We provide the first physiological evidence that ITD curves can be shifted by inhibition in the manner described in the Brand model. We showed that, for several leading inhibitory delays, the spike rate at zero ITD fell below $50 \%$ of the control. At longer ITDs, the spike rate increased at longer ITDs and could stay elevated for 100-300 $\mu$ s changes in ITD and then declined over the next 200-400 $\mu \mathrm{s}$, giving the ITD curve a "domed" appearance.

One could argue that the dome-shaped ITD curves are a result of synaptic depression i.e. the first activation of inhibition at zero ITD is the strongest and therefore suppresses action potentials. Then for each subsequent activation, the synaptic strength has decreased and the firing rate increases at longer ITDs. Synaptic depression is thought to occur from the depletion of synaptic vesicles resulting from repetitive stimulation. This most likely is not the case, as we did not observe a significant amount of depression, as measured from the amplitudes of IPSPs at the $100 \mathrm{~Hz}$ frequency of stimulation that we used.

We also show that inhibitory inputs from the MNTB could completely suppress generation of action potentials. The complete or near complete suppression of action potentials most often occurred when the inhibitory input was activated at the start of the excitatory current injection. An IPSP that starts at the same time as the excitatory input has the most influence over it, because the entire IPSP can interact with the EPSP. 
Overall, there are two main specializations that work to shape the neural response of MSO cells to ITDs, voltage gated potassium channels and inhibitory inputs. The voltage gated potassium channels work to decrease the coincidence window, thus sharpening ITD curves. Inhibitory inputs could work in several ways. Inhibition could further sharpen ITD curves, could suppress action potential generation at unfavorable delays, or shape the response so that the peak of the ITD falls away from zero ITD. Both the potassium channels and inhibitory inputs work in a delicate balance to generate sensitivity to ITDs. 


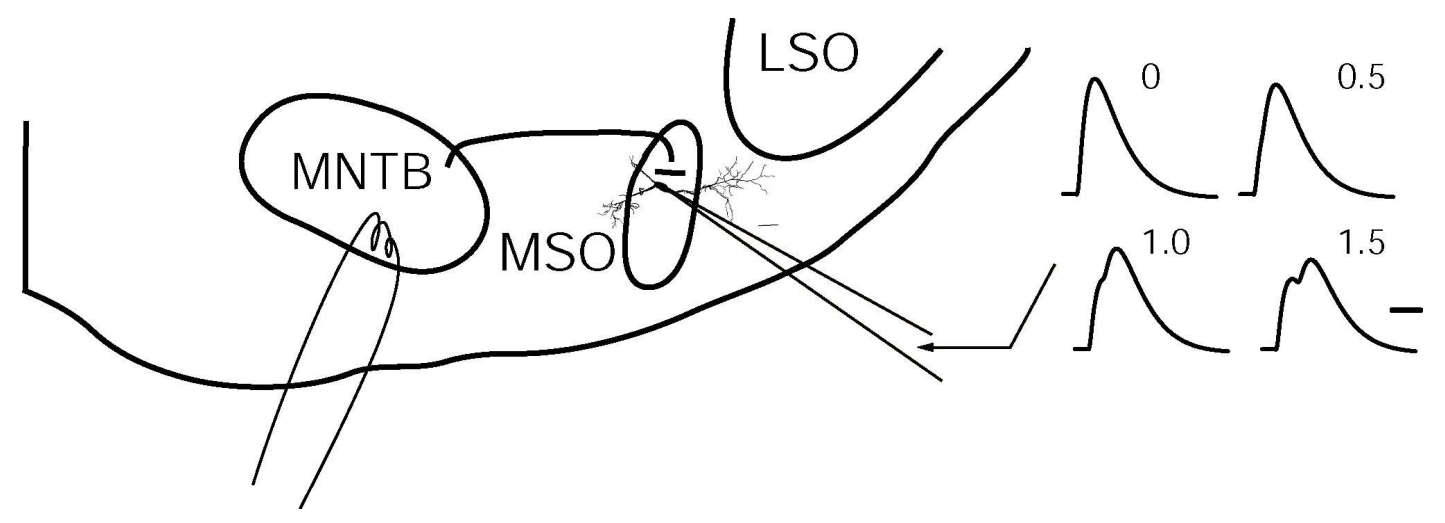

Figure 1. Semi-schematic of the superior olivary complex of the gerbil.

Whole cell recordings were made from the principal cell column of the MSO, while injecting simulated ITD current injections through the recording pipette. Waveforms representing current injections simulating $0,0.5,1.0$ and $1.5 \mathrm{msec}$ ITD are shown on the right. The role of the inhibitory inputs to the MSO in ITD coding was studied by stimulating MNTB cells with bipolar electrodes. 


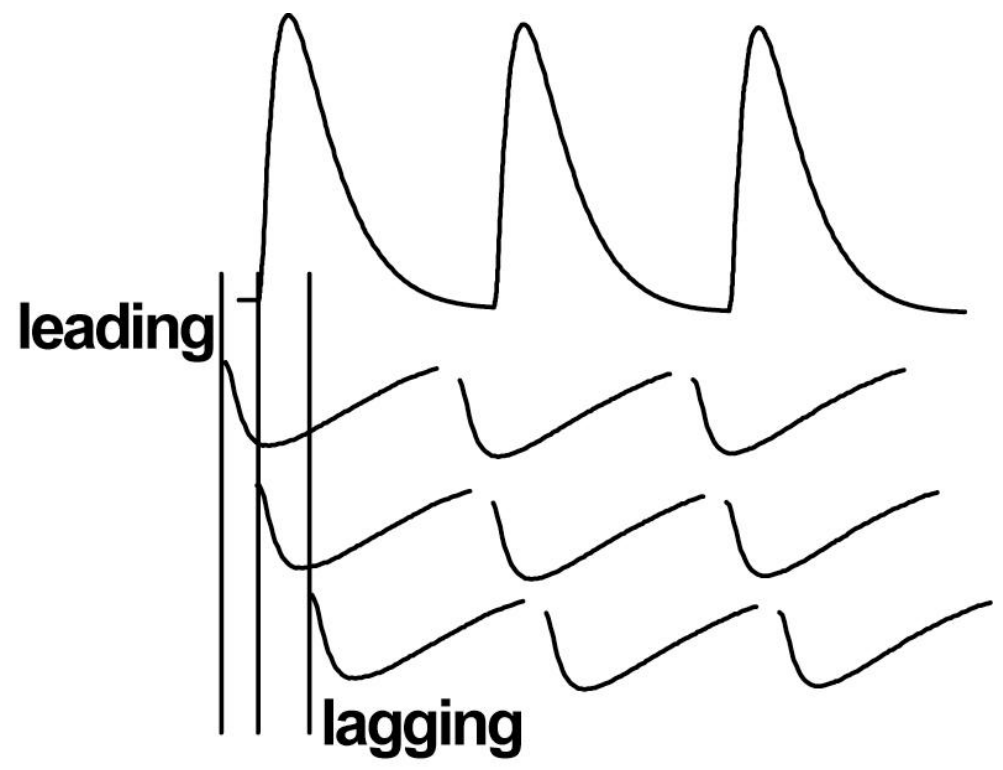

Figure 2. Stimulation protocol used to study the effects of varying the onset of inhibition during simulated ITD current injections.

IPSPs were elicited in the MSO by shock of the MNTB (bottom three traces). The effects of an IPSP arriving prior to excitation ("leading") were tested by shocking the MNTB up to $5 \mathrm{~ms}$ prior to the start of the excitatory current injection through the recording pipette (top trace). The effects of IPSPs arriving following excitation ("lagging") were tested by shocking the MNTB up to 2 ms following the start of the excitatory current injection. 


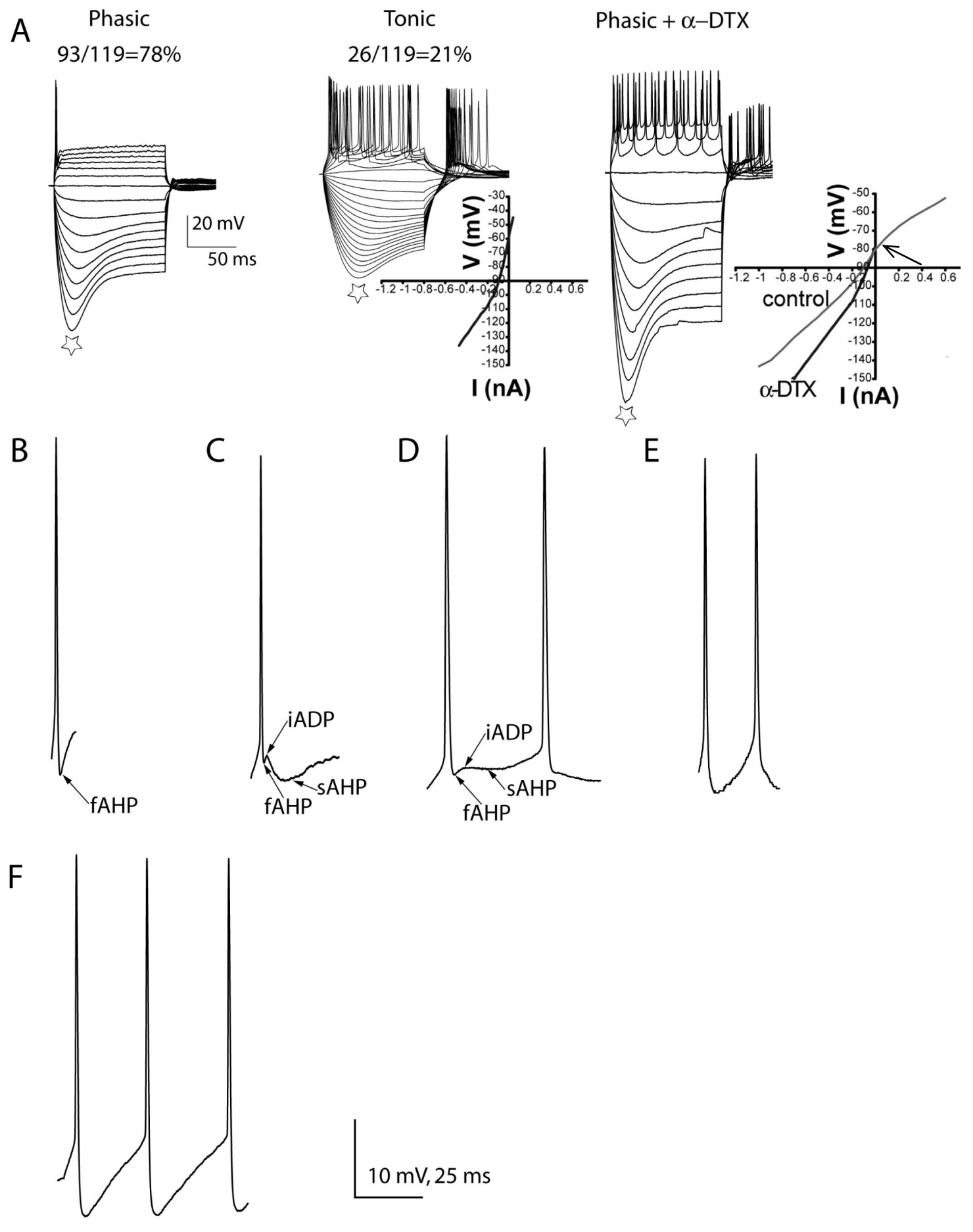

Figure 3. Voltage responses to hyperpolarizing and depolarizing current injections.

A. Most of the neurons recorded from the principal cell column after PD 11 
responded with 1-3 action potentials upon sustained depolarizing current injection (93 out of 119; labeled phasic). The remainder of cells fired repetitive action potentials during the current pulse (26 out of 119; labeled tonic). The inset depicts the IV curve for the tonic response shown. When the potassium channel blocker, $\alpha$-DTX was superfused into the bath, the phasic cell pictured on the left became tonically active (Phasic $+\alpha$-DTX; inset depicts IV curve prior to and following application of $\alpha-D T X)$. B. Action potential from the same phasic neuron depicted in $A$. Phasic neurons exhibited afterhyperpolarizations that were fast (1-2 ms in duration; labeled fAHP). C and D. Action potentials from the tonic neuron depicted in A. In six out of 26 tonic cells, afterhyperpolarizations exhibited a fast afterhyperpolarization (fAHP) followed by an intermediate afterdepolarization (iADP) then a slow afterhyperpolarization (sAHP). The multiple component afterhyperpolarizations were not observed when $\alpha$-DTX was added to phasic neurons $(E)$ or in other tonic neurons $(F)$. 

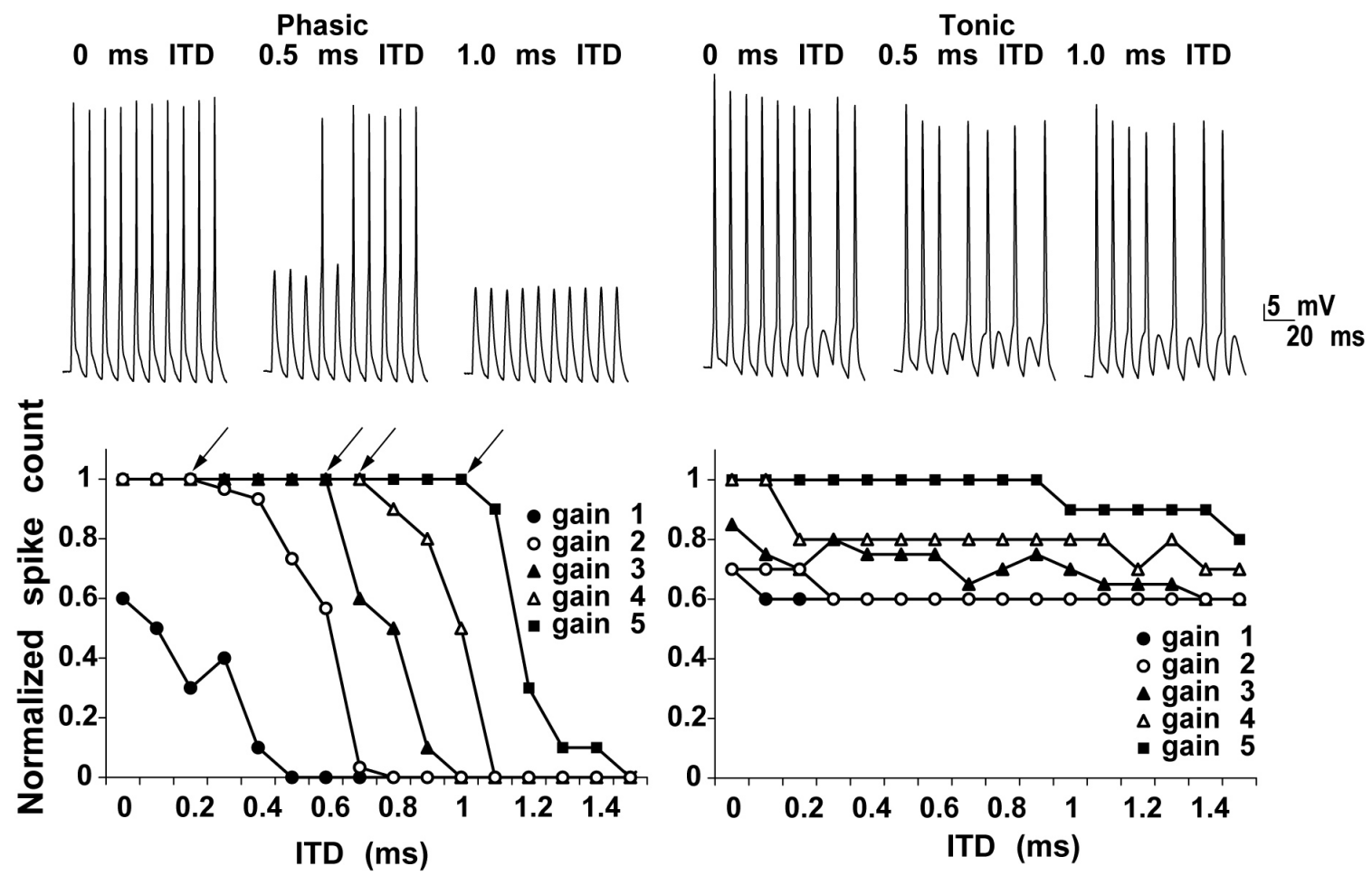

Figure 4. Phasic neurons have steeper ITD curves than tonic neurons.

Composite EPSP waveforms simulating ITDs were injected into the soma of MSO cells at $100 \mathrm{~Hz}$ in increments of $100 \mu \mathrm{sec}$ ITD. Top: Example voltage responses to $0,0.5$ and $1.0 \mathrm{msec}$ simulated ITD are shown for phasic and tonic cells. Bottom: Normalized spike counts versus simulated ITD. Phasic cells (left) had steep ITD curves, whereas tonic cells had little or no sensitivity to ITDs (right). For simplicity, stimulus amplitude (gain) is numbered in increasing magnitude; numbers only depict the sequence of gain values and do not refer to the absolute value of the stimulus. Arrows point to corner ITDs. 
Figure 5A

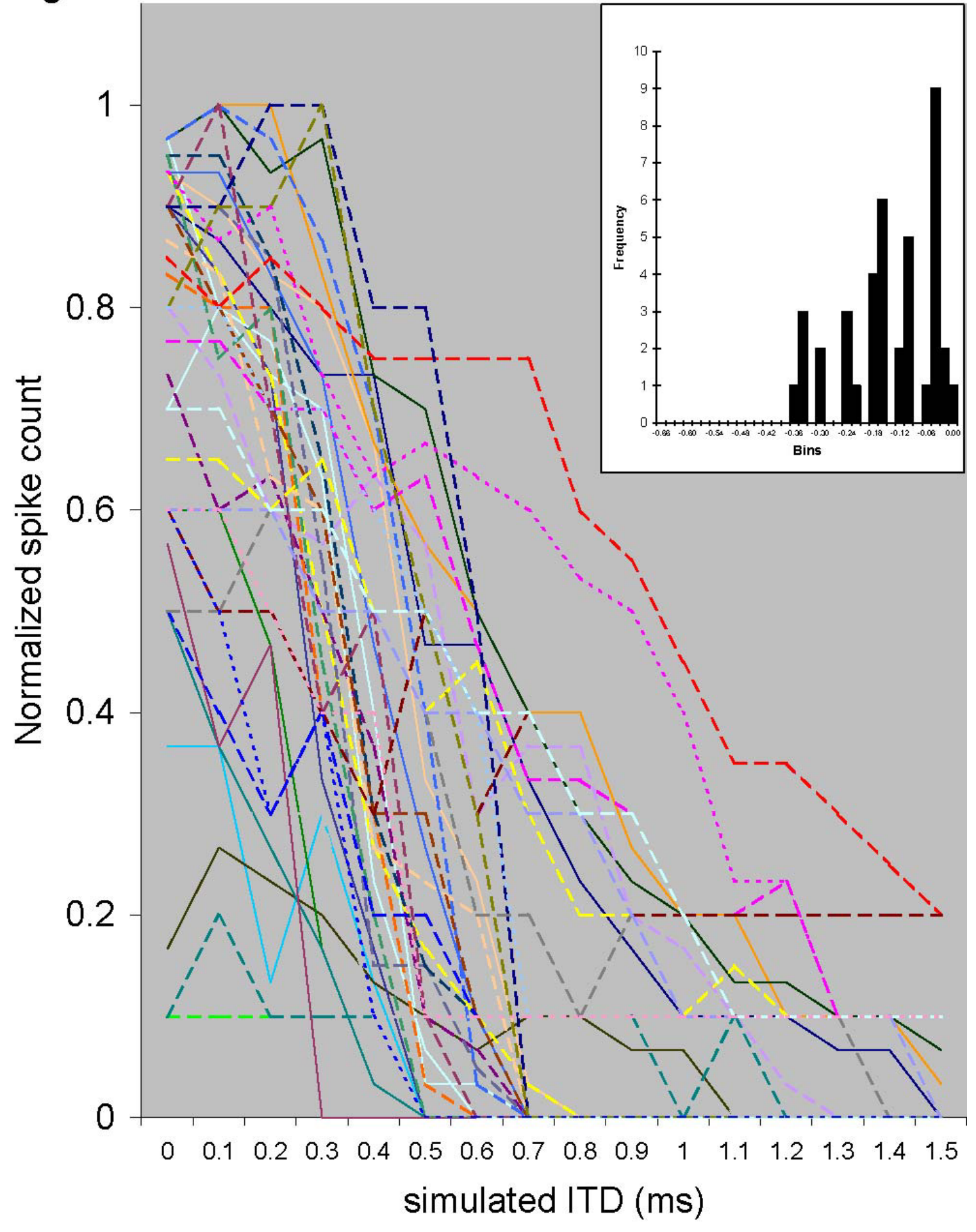


Figure 5B

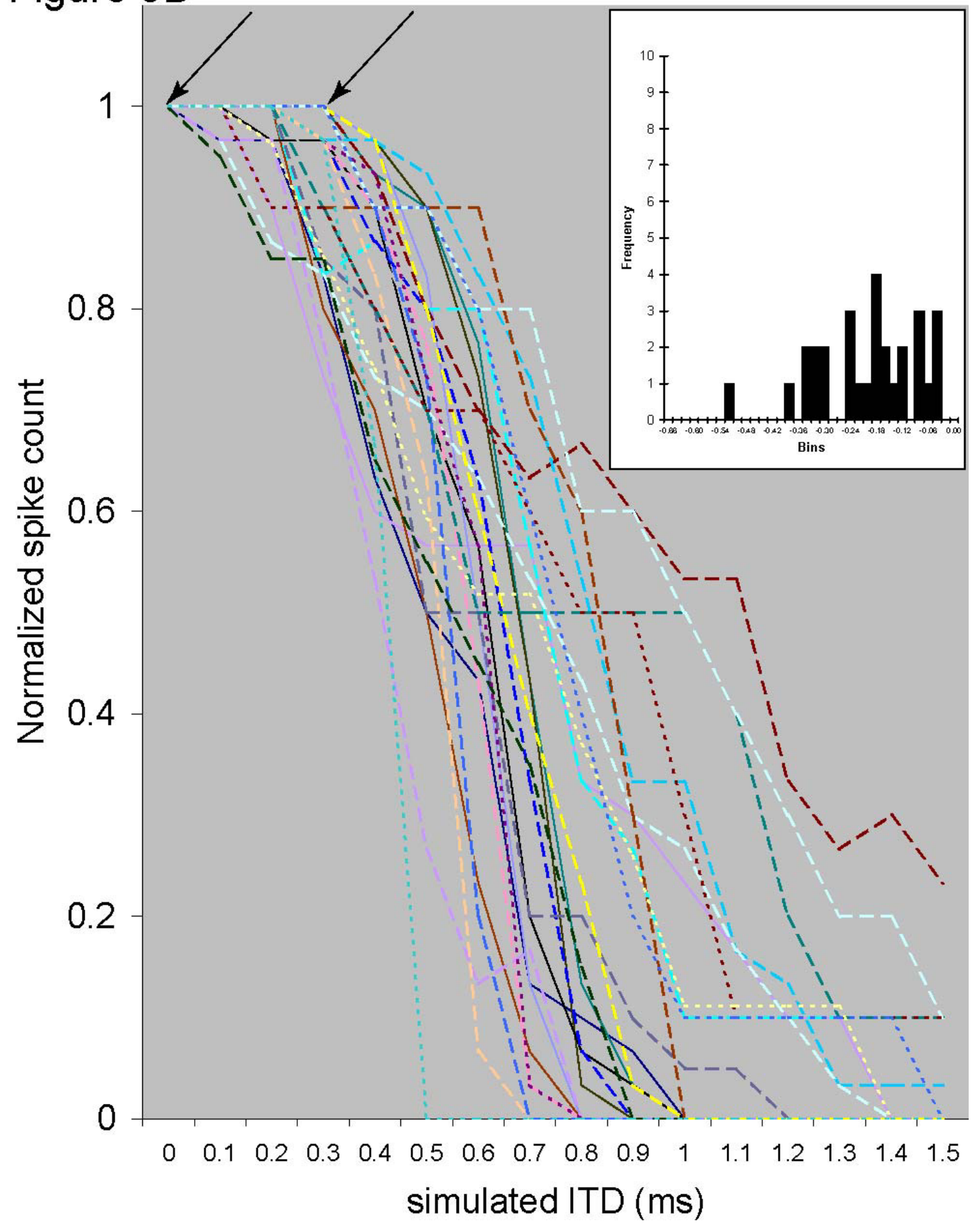


Figure 5C

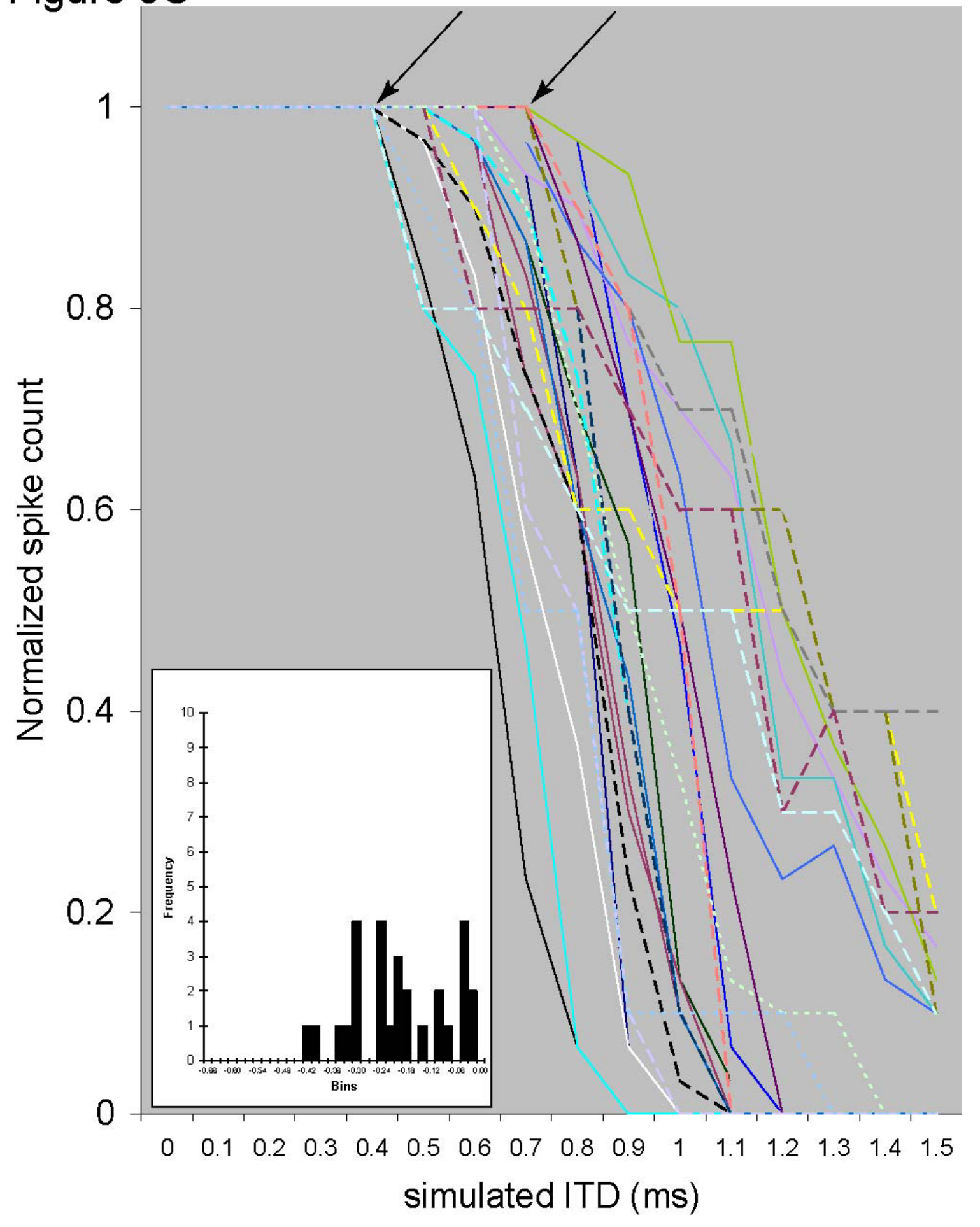


Figure 5D

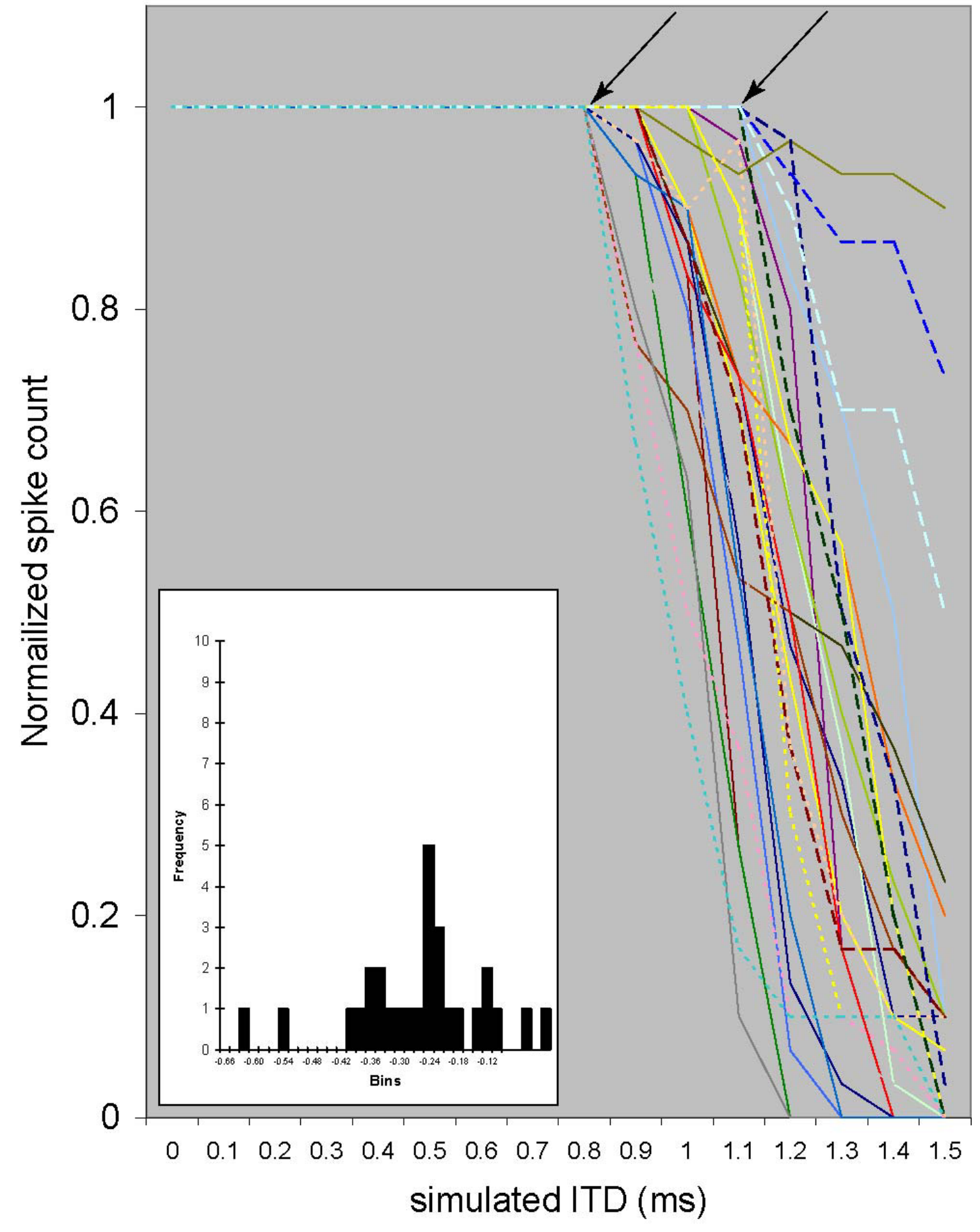


Figure 5E

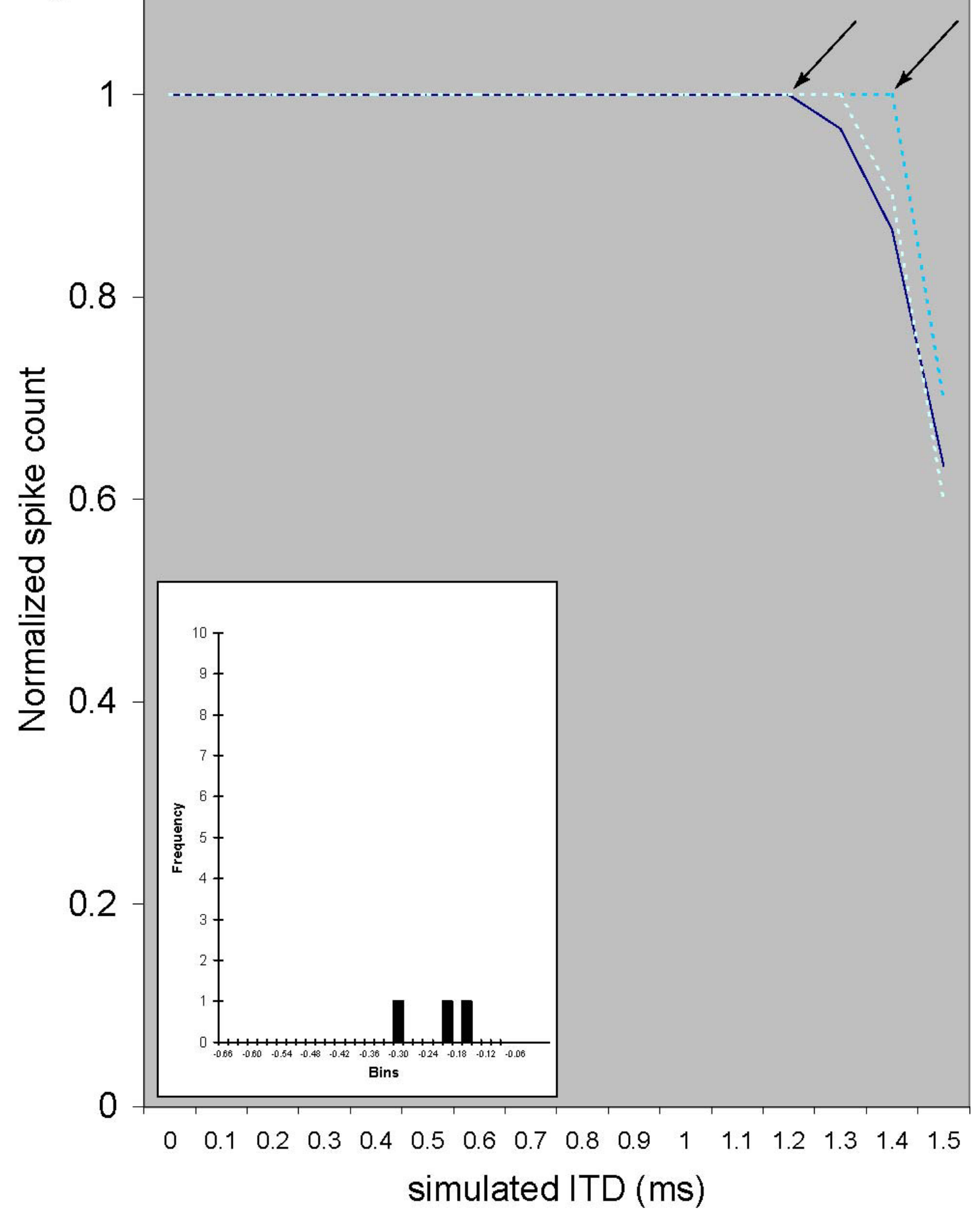


Figure 5A-E. Graphs of control simulated ITD curves from 40 phasic cells.

Normalized spike counts versus ITD for five groups based upon corner ITD. A: Voltage recordings that did not reach entrainment at zero ITD (below 1.0 on the ordinate) were grouped as "below" entrainment (Figure 5A; $n=40$ ITD curves). Inset represents histogram of the slopes of the ITD curves between .8 and .2 spike counts (80 to 20 slope; bin width 0.02 counts per $100 \mu \mathrm{secs}$ ). When the 80 to 20 slope was unable to be calculated, for example if the spike count did not fall below 0.2 , the steepest part of the slope (as determined by eye) was recorded. B: ITD curves from stimuli that elicited entrainment to corner ITDs of 0 to $0.3 \mathrm{~ms}$ (corner ITDs marked by arrows; $n=29$ curves). C: ITD curves from stimuli that elicited entrainment to corner ITDs of 0.4 to $0.7 \mathrm{~ms}$. ITD curves from stimuli that elicited entrainment to corner ITDs from 0.8 to $1.1 \mathrm{~ms}$ ITD. E. ITD curves from stimuli that elicited entrainment to corner ITDs from $1.2 \mathrm{~ms}$ to $1.5 \mathrm{~ms}$ ITD. 


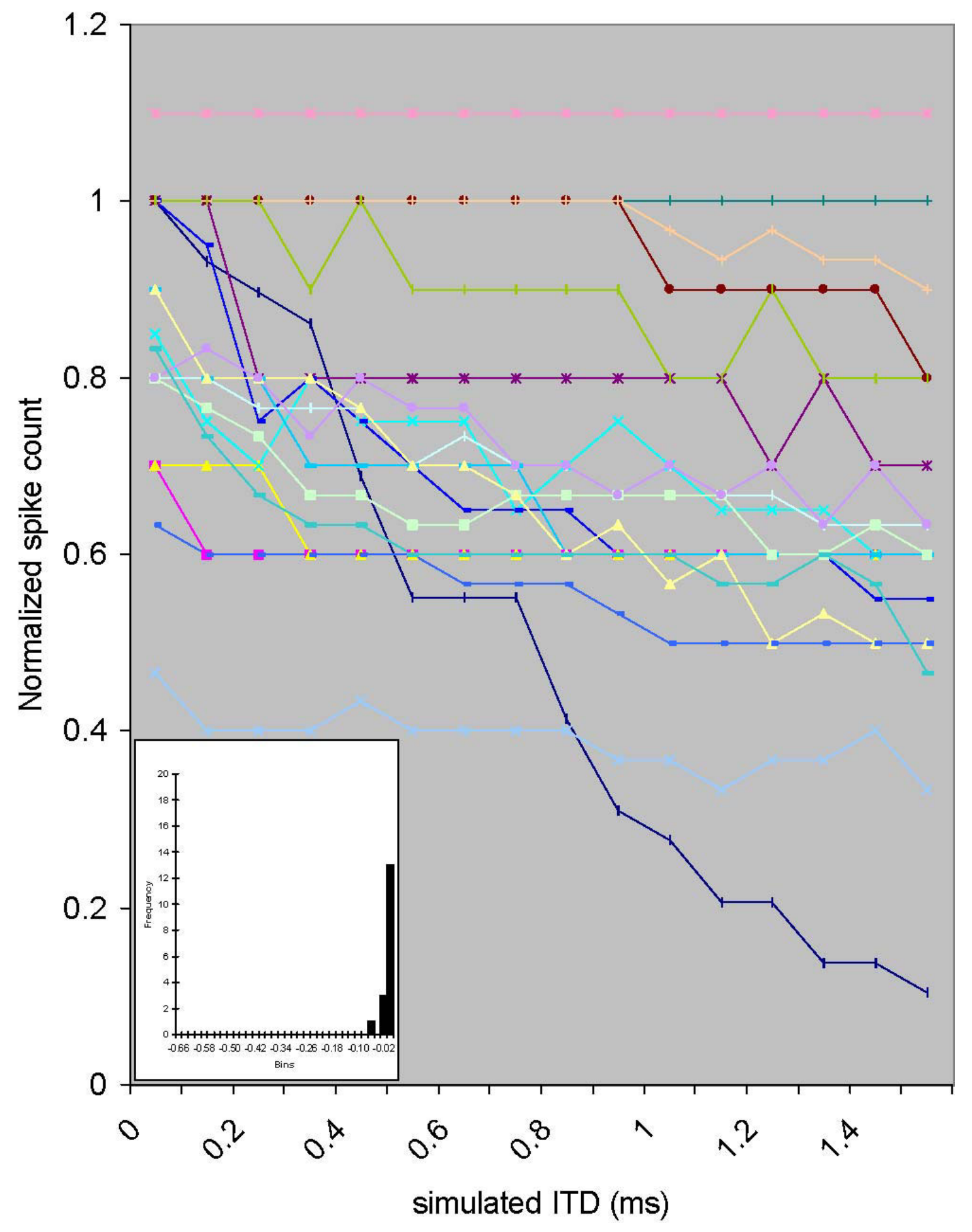

Figure 6. Representative ITD curves from tonic neurons.

We tested the sensitivity of tonic neurons to changes in ITD by injecting simulated ITD current injections identical to those injected into the phasic 
neurons. The above depicts the 19 control ITD curves from 13 tonic neurons. 


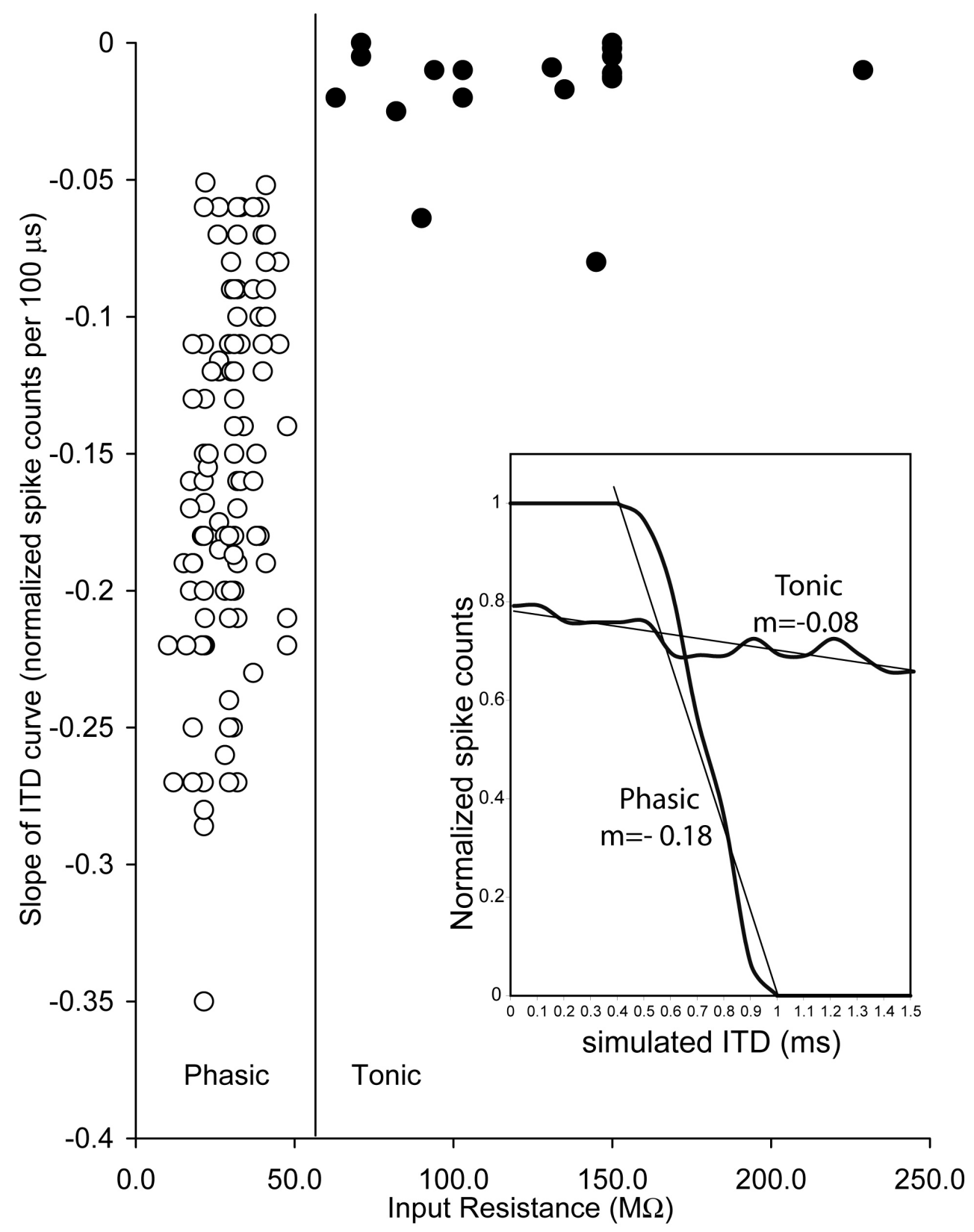

Figure 7. Phasic neurons have low input resistances and steep ITD curves whereas tonic neurons have high input resistances and shallow ITD curves.

The inset at right shows example ITD curves for a phasic and tonic neuron. The slope of the ITD curve for phasic neurons was taken between the corner ITD and zero rate. The entire curve was used to calculate the slope of tonic neurons. 


\section{Phasic}

\section{Tonic}
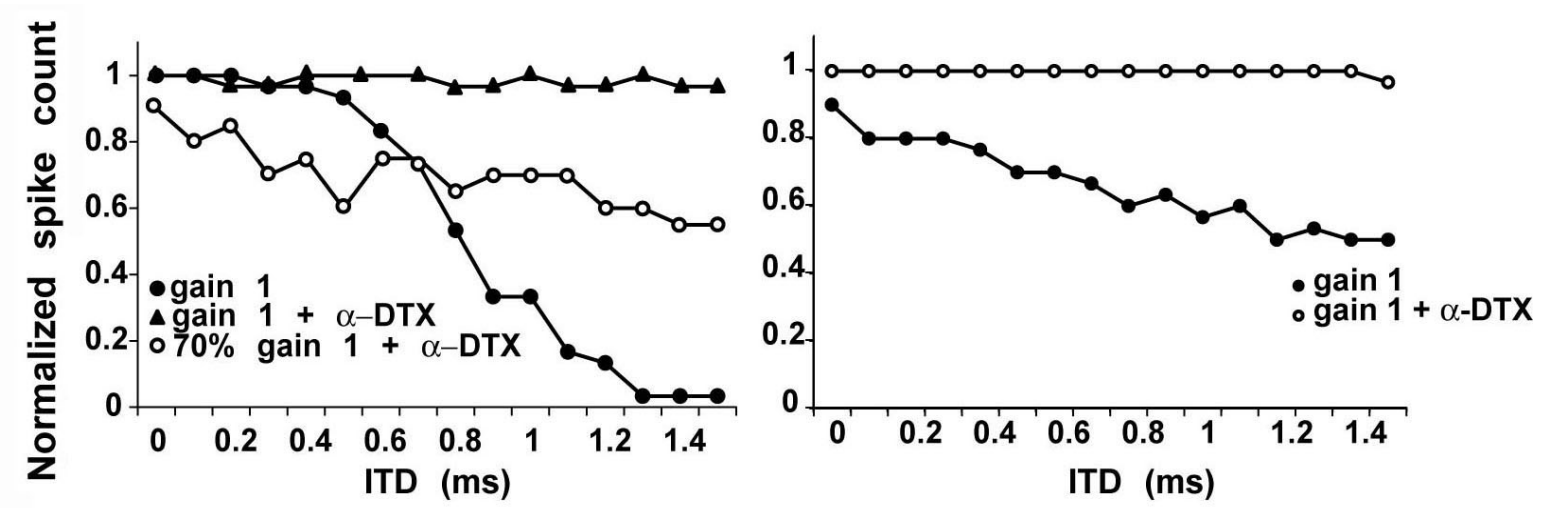

Figure 8. Normalized spike counts in response to simulated ITD for phasic (left) and tonic (right) neurons before and following application of $\alpha$-DTX.

Left: The sensitivity to ITDs of the phasic cell (filled circles) was abolished after application of $100 \mathrm{nM} \alpha$-DTX to the bath (triangles). Decreasing the gain by $70 \%$ of the control partially restored sensitivity to ITDs (open circles). Right: Tonic cells had little sensitivity to ITDs that was completely eliminated upon application of $\alpha-D T X$. 

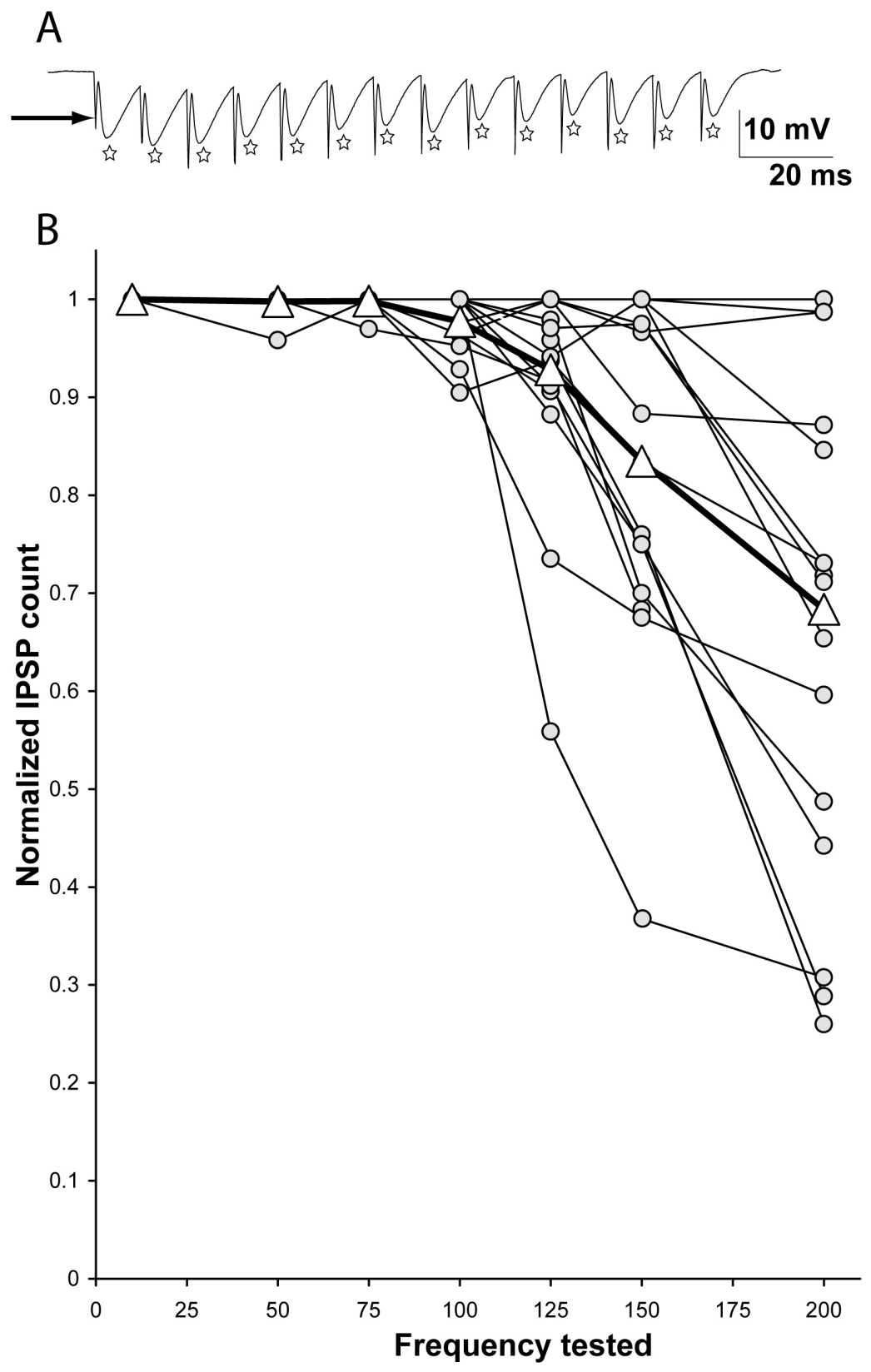

Figure 9. The ability of MSO cells to follow MNTB stimulation with IPSPs decline with increasing frequency.

A. Example IPSPs (stars) recorded from an MSO cell in response to $100 \mathrm{~Hz}$ stimulation of the MNTB. The arrow points to the stimulation artifact. B. The normalized IPSP counts from single cells are represented by circles, whereas the average across all cells are represented as triangles. 

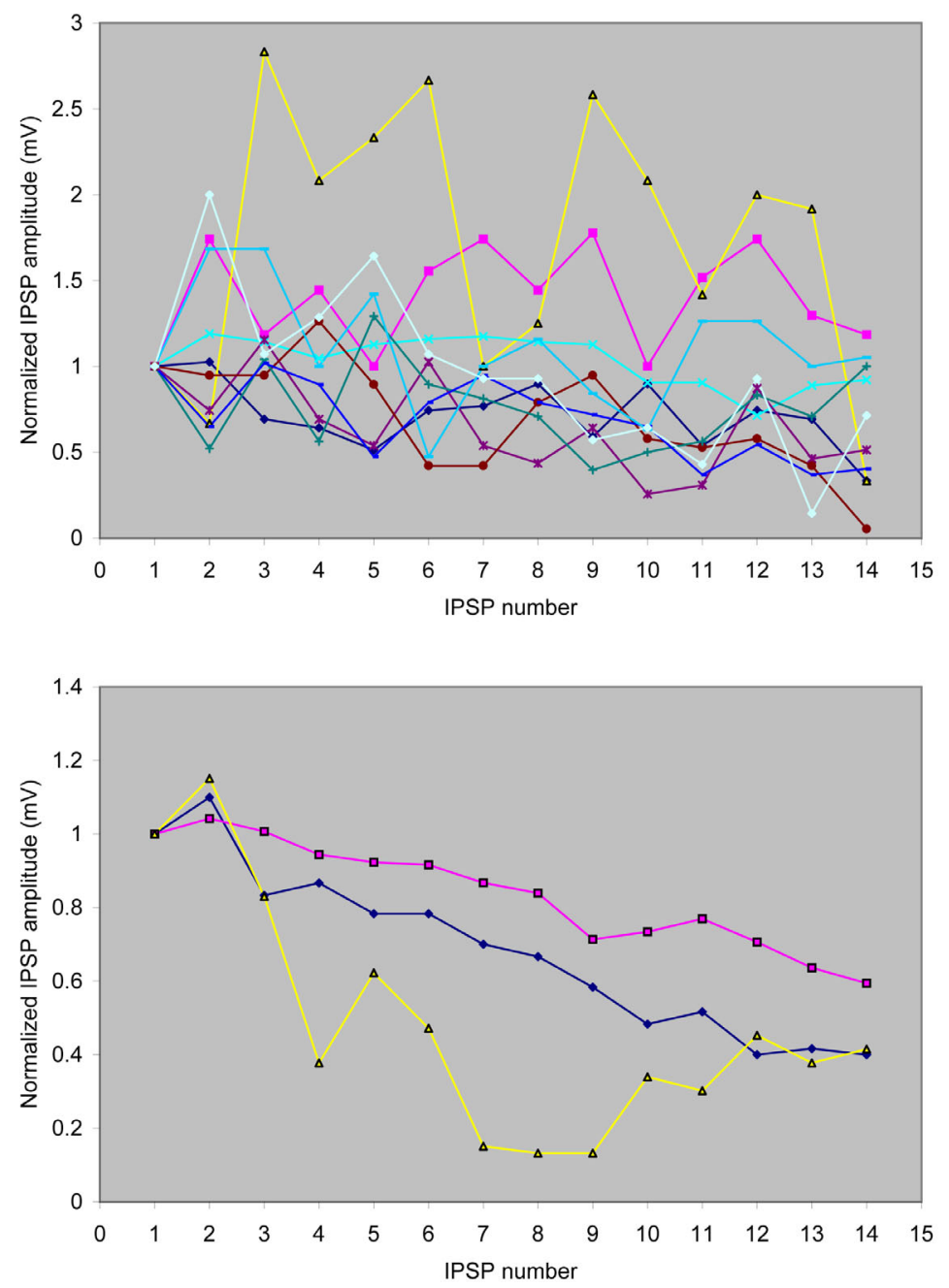

Figure 10. Normalized IPSP amplitude versus number in the train.

We tested for the effects of synaptic depression by recording IPSPs from 14 shocks (at $100 \mathrm{~Hz}$ ) to the MNTB. Most cells (top) displayed little to no synaptic depression whereas three cells displayed marked synaptic depression and were therefore excluded from analysis. 


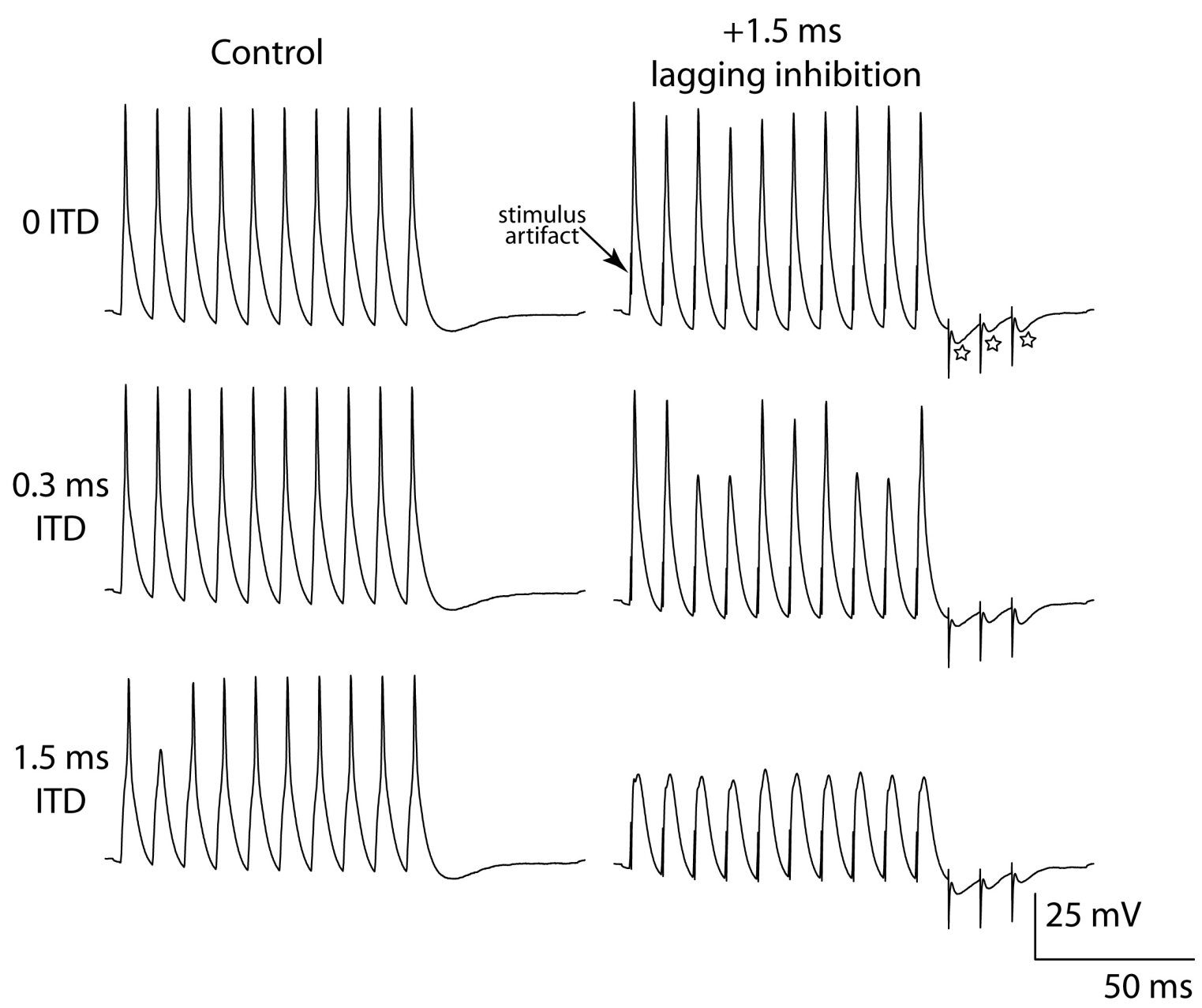

Figure 11. Voltage recordings of MSO cells injected with excitatory current alone and conditions with combined excitation and inhibition.

Control. Responses of MSO to simulated ITD current injections. At 0 and $0.3 \mathrm{~ms}$ ITD the current injection stimulus was of sufficient magnitude to elicit entrainment with action potentials to each cycle of the stimulus. At long ITDs the entrainment declines to 9 out of ten cycles of the stimulus. +1.5 ms lagging inhibition. We tested the effects of lagging inhibition on excitation by stimulation of the MNTB (stimulus artifact marked by arrow). At 0 ITD the current injection stimulus was strong enough to elicit entrainment; however at longer ITDs the spike rate drastically declined compared to control causing complete suppression at $1.5 \mathrm{~ms}$ ITD. We tested the viability of the inhibitory afferents by three test stimuli following each simulated ITD (stars in top trace). 

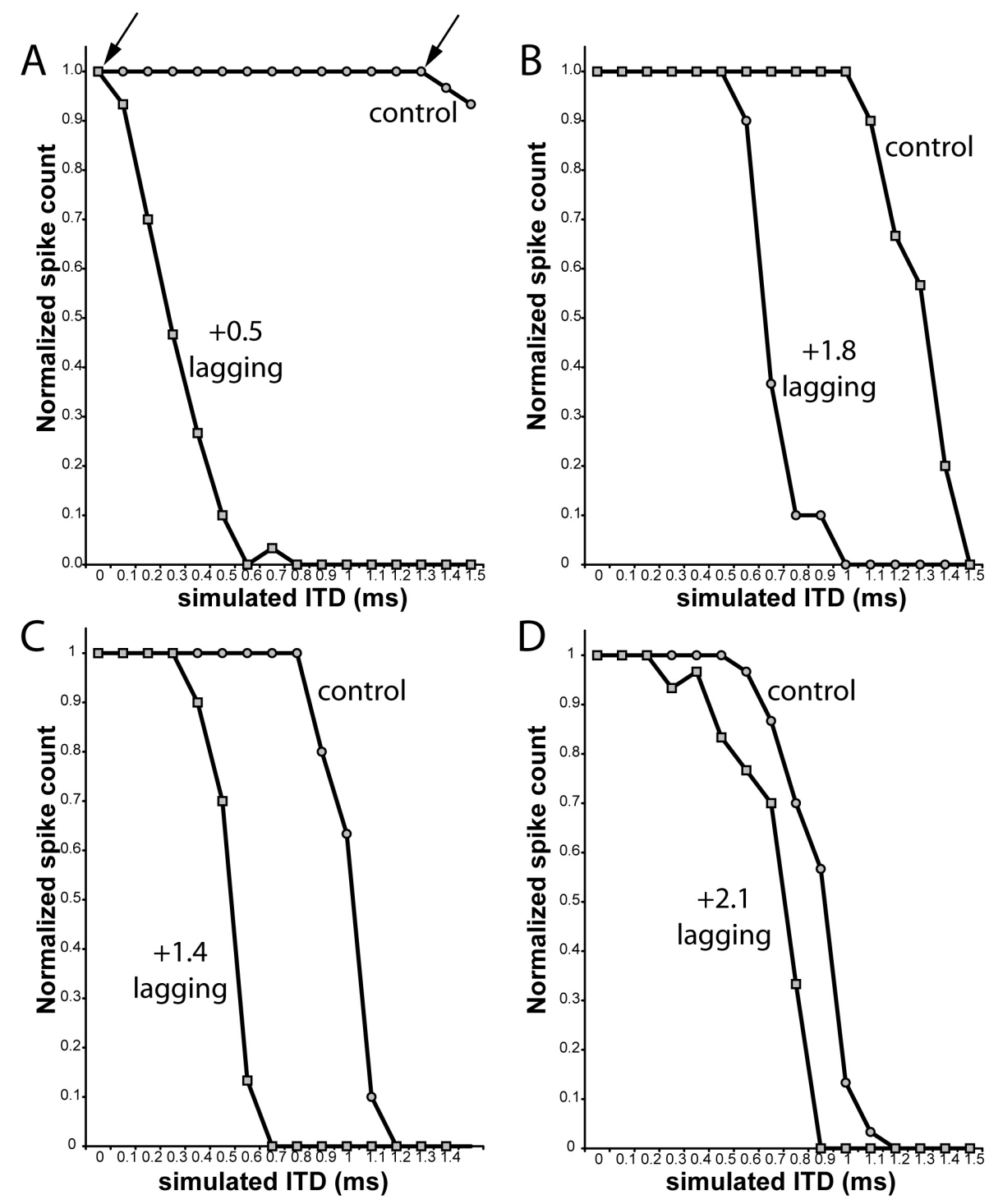

Figure 12. ITD curves of control and lagging inhibition.

Lagging delays typically shifted the corner ITDs to shorter ITDs. The corner ITDs in $A$ are depicted by arrows. The shifting of the corner ITD ranged from very large shifts $(1.3 \mathrm{~ms}$ in $A)$ to more modest shifts $(0.3 \mathrm{~ms} D)$. Shifting of corner ITDs differed from gain to gain and between cells. 


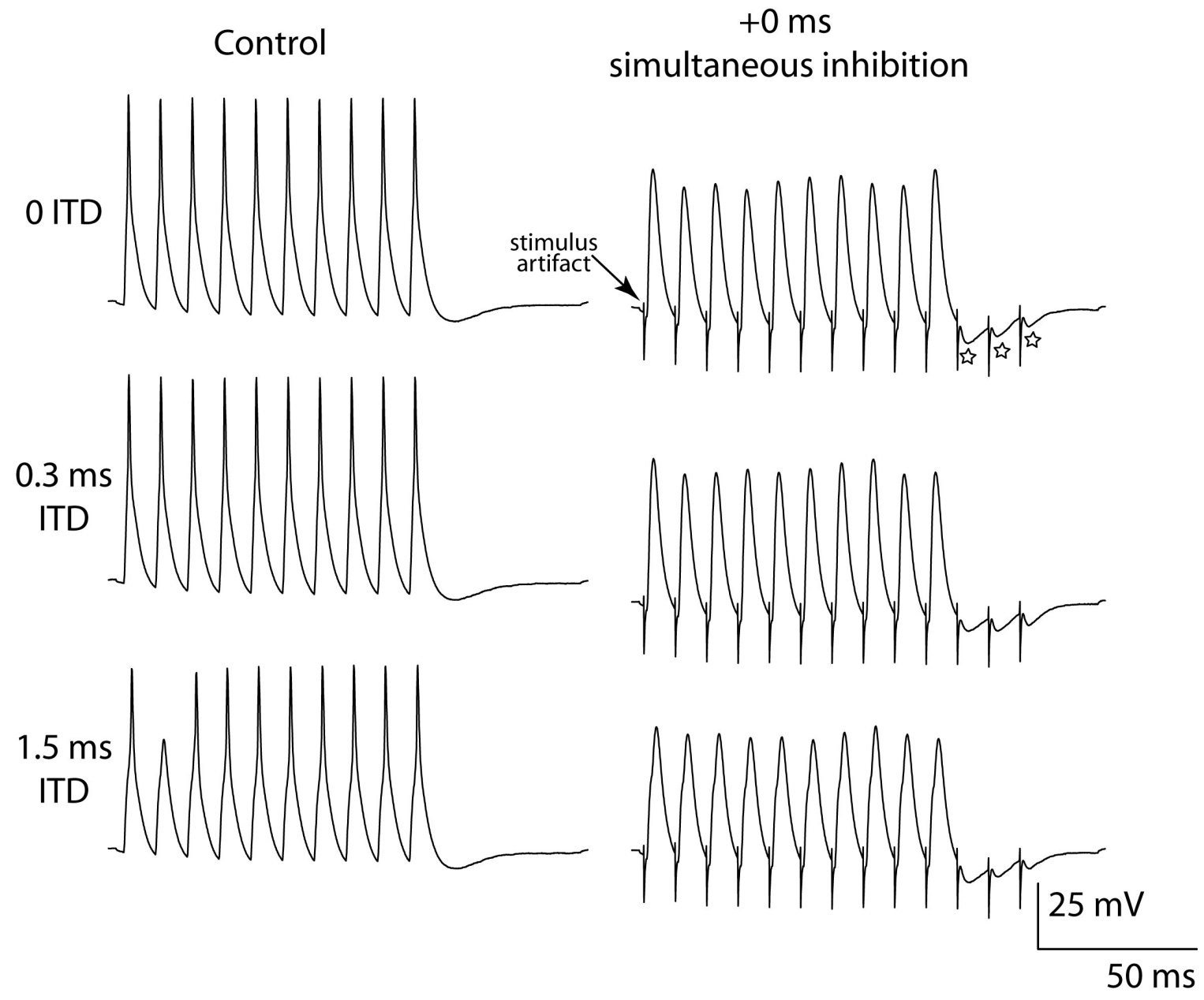

Figure 13. Voltage recordings during control and simultaneous onset of inhibition conditions.

Control. Same recordings as those depicted in Figure 11. 0 ms simultaneous inhibition. In this example and for many cells, stimulation of the MNTB caused a complete suppression of action potentials at all ITDs. 

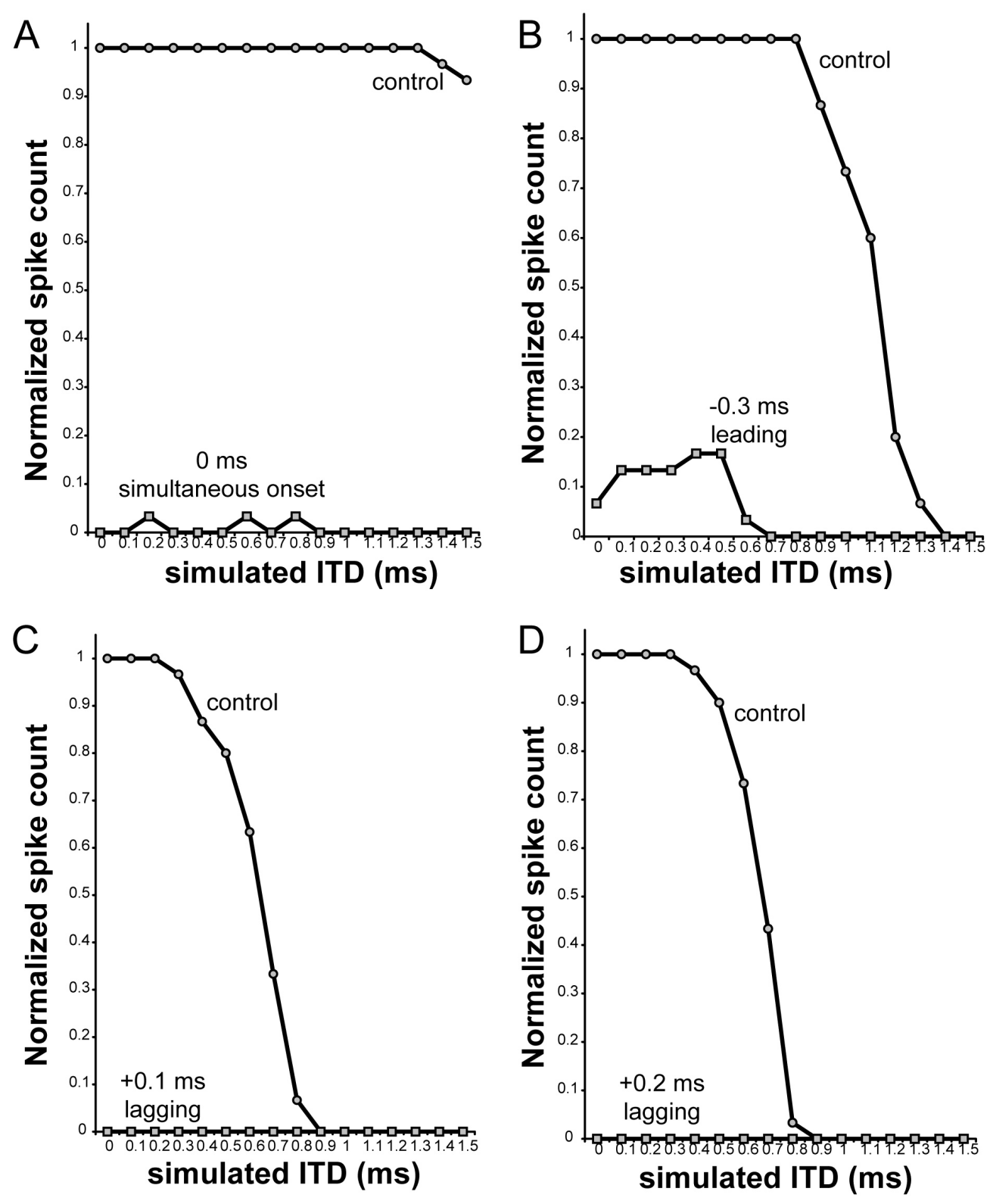

Figure 14. Simultaneous onset of inhibitory inputs decrease the normalized spike rate of ITD curves.

Activation of inhibitory inputs at or near the start of the excitatory current injection caused significant suppression of action potentials at all ITDs. 


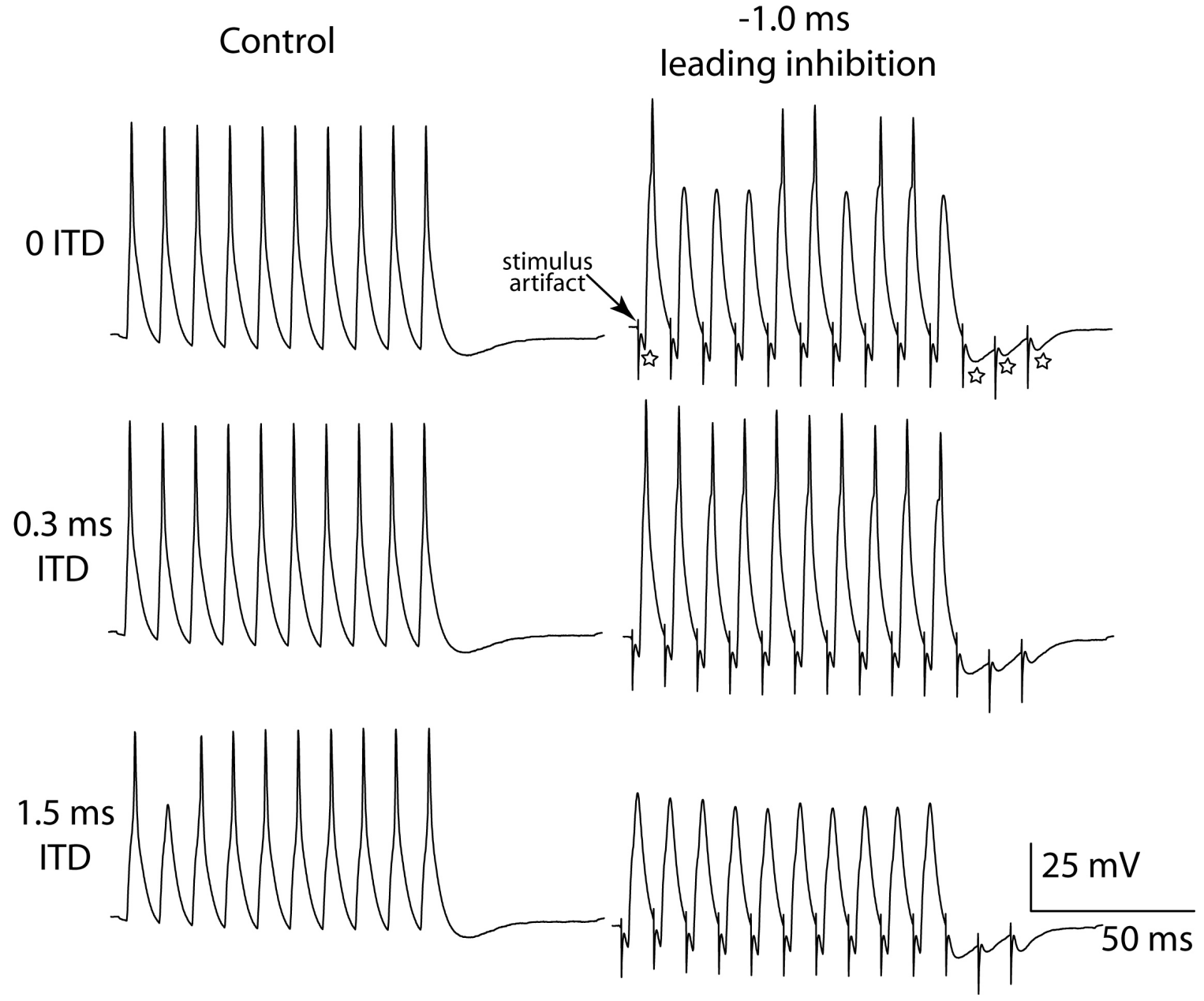

Figure 15. Voltage recordings during control and leading inhibition conditions.

Control. Same recordings as those depicted in Figures 11 and 13. -1.0 leading inhibition. In this example, leading inhibitory activation caused a hyperpolarization that preceded the excitatory current injection (star below stimulus artifact arrow). At 0 ITD inhibition suppressed 5 out of 10 action potentials. At $0.3 \mathrm{~ms}$ ITD the spike count increased to 10 actions and then 1.5 ms ITD the spike count decreased to zero. 

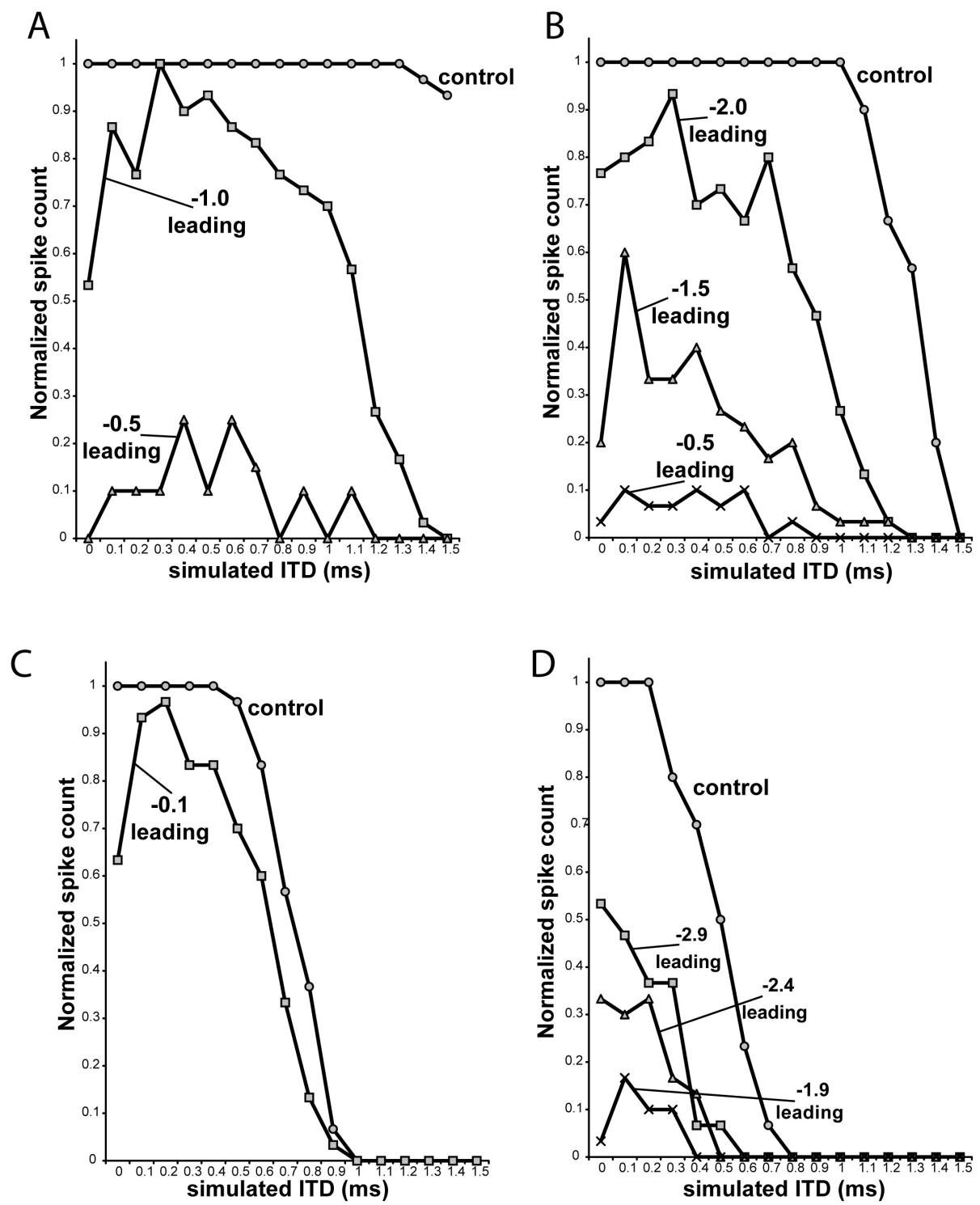

Figure 16. Effects of leading inhibition on the coding of ITDs.

Leading inhibitory activation caused two separate response types. In some cases action potentials were suppressed at short ITDs, then spike counts increased at longer ITDs, then decreased again at very long ITDs. 

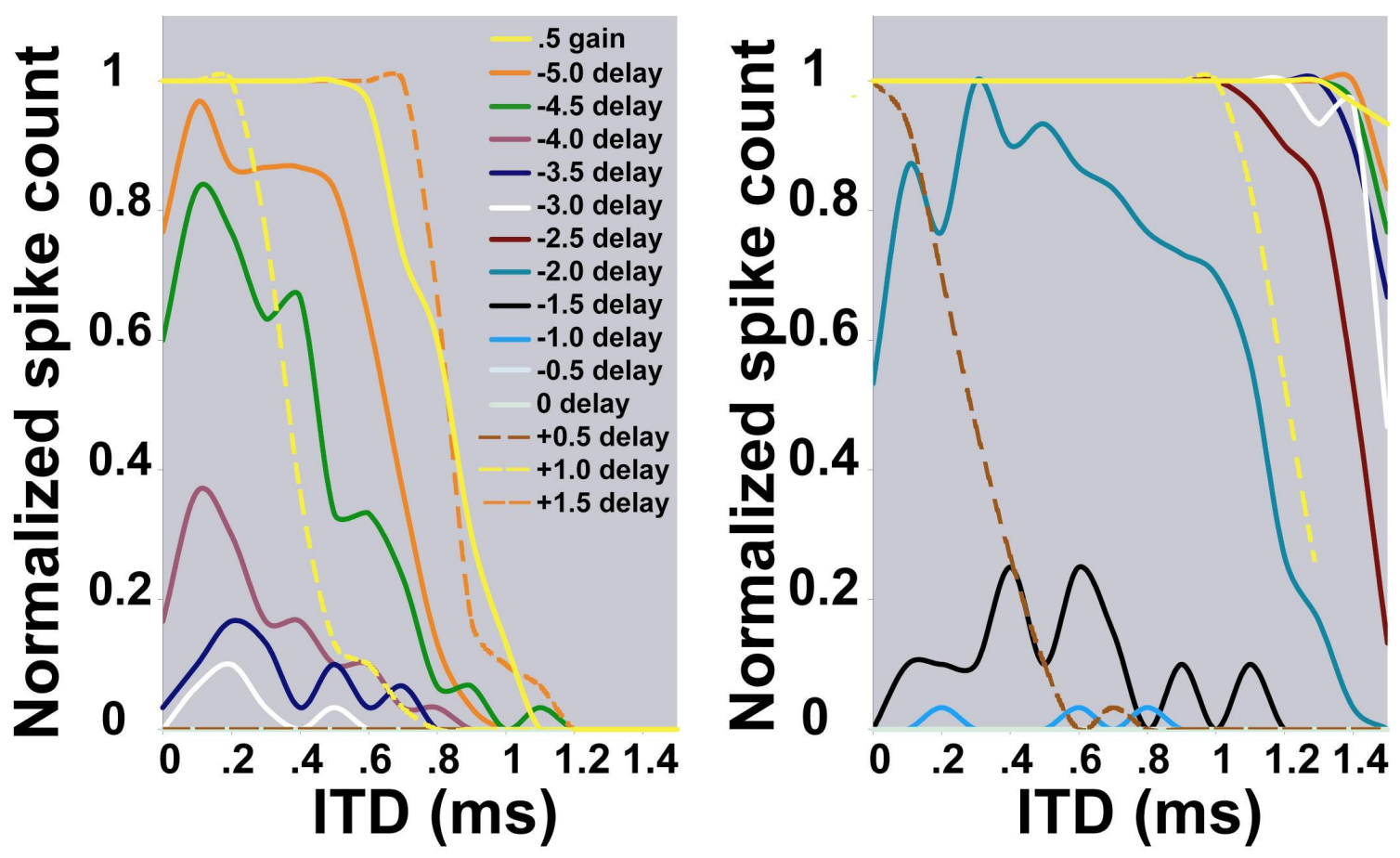

Figure 17. Composite ITD curves for two cells.

ITD curves showing normalized counts of action potentials at different simulated ITDs both with (solid yellow curve) and without activation of inhibitory inputs (remaining curves). Left. Simulated ITD current injections in control conditions (no inhibition) elicited entrainment of action potentials to $0.5 \mathrm{~ms}$ ITD then a sharp decline in spike counts to 0 ITD at $1.1 \mathrm{~ms}$ ITD. When inhibitory inputs were activated prior to excitation, $(-5.0$ to $-3.0 \mathrm{~ms})$ spike counts were attenuated and ITD curves exhibited "dome" shaped appearances. When inhibitory inputs were activated at or very near the start of excitation $(-2.5$ to $+0.5 \mathrm{~ms})$ action potentials at all ITDs were suppressed. When inhibitory inputs were activated following excitation (dotted curves; $+1.0 \mathrm{~ms}$ ) ITD curves shifted corner ITDs thereby sharpening ITD curves. Right. When higher gain excitatory current injections were used action potentials were generated at longer ITDs (solid yellow curve). Inhibitory inputs could however generate dome-shaped curves during leading inhibition (-2.0 to $-1.0 \mathrm{~ms})$ and could nearly or completely suppress action potentials at or near the start of excitation $(-1.0$ to $0 \mathrm{~ms})$. Lagging inhibition shortened corner ITDs (+1.0 to $1.5 \mathrm{~ms})$. 
Chapter 6: Suggestions and General Discussion

The use of ITDs by the auditory system requires accurate encoding of the temporal features of auditory stimuli. Humans are able to detect differences between sound sources separated by a minimal audible angle of only one degree which corresponds to an ITD of only a few microseconds (Yost 1974) for locations directly in front of the head (Zwislocki and Feldman 1956). The aim of this dissertation was to investigate the cellular mechanisms found along the auditory pathway that underlie complex perceptual phenomena such as localizing sounds by the use of such small ITDs. By using electron microscopy and a combination of neuronal modeling and electrophysiology we investigated some of the cellular specializations that cells of the lower auditory brainstem employ to accurately encode temporal information. In this section the relevance of the experimental results to sound localization in vivo is considered, along with suggestions for further studies.

\section{Large nerve terminals and MACs}

In chapter 2 we made observations of the ultrastructure of the calyx of Held, and the distinct organelle arrangements found within the calyces. The globular bushy cell axon that leads into the calyx of Held terminal exhibits some of the fastest recorded spike rates, in that it can entrain to stimuli at rates approaching $1 \mathrm{kHz}$ (Joris et al. 1994a). Such high activity rates indicate that calyces must contain specific cellular features that assist in vesicle fusion, synaptic vesicle membrane recycling and membrane adherence. We identified in the calyx of Held a conglomerate of highly arranged presynaptic organelles, which we named the MAC. Our reconstructions of serial section electron micrographs demonstrate that MACs exhibit an extremely close spatial relationship to the sites of synaptic vesicle docking called active zones. The proximity of MACs to active zones is consistent with a role in the life cycle of a synaptic vesicle. We offer several hypotheses in Chapter 2 for the function of MACs in synaptic vesicle transmission and recycling and and the reader is referred to that section. 
Much work remains to be completed to further dissect the anatomy and physiology of MACs. Recently, other members of our group in a collaborative effort with the University of San Diego have used the intermediate voltage electron microscope (EM) to gain a more detailed look at the anatomy of MACs. One advantage of using the intermediate voltage EM is that instead of using ultrathin sections $(60-70 \mathrm{~nm})$ as used in a conventional EM, thicker sections ( $\sim 500 \mathrm{~nm})$ can be used and thereby decrease the likelihood of damaging serial sections. A more important advantage is the $5 \mathrm{~nm}$ spatial resolution through the thickness of the tissue specimen, which exceeds the 30-70 nm resolution achievable using standard ultrathin sectioning techniques. The intermediate EM is currently being used to examine the three dimensional structure of the hypothesized vesicle recycling zone of the MAC, called the vesicular chain. Eventually, the microscope could also be used to investigate all of elements the MAC.

The proteins that make up the components of the MAC are unknown. We suggest that studies that combine the use of electron microscopy and immunocytochemistry be undertaken to determine the nature of the punctum adherens, mitochondrial plaque and filaments. Knowledge of these components will provide additional clues to their roles in calyx function. The cadherin proteins may link to the actin cytoskeleton and be under dynamic control by second messenger systems. The members of the cadherin family may make up the proteins of the punctum adherens as they are often found localized near synaptic junctions (Uchida et al., 1996) and is consistent with our find that the puncta adherentia often merged with the active zones. Immunocytochemical markers for cadherins could be used as a first step to identify the proteins in the punctum adherens. Also, physiological studies of calyces implicate MAC components, albeit indirect, to specific functions. For example, a recent study showed that vesicle recruitment in the calyx of Held is dependent upon actin and molecules of ATP (Sakaba and Neher, 2003). The mitochondria of MACs likely supply the necessary ATP for recruitment and their filaments may be composed of actin. A detailed immuncytochemical study would be helpful to confirm these claims. 
There are no direct studies that have investigated the physiology of MACs. As a possible first step, studies that replicate the classical labeling studies of Heuser and Reese that examined vesicular recycling at the neuromuscular junction (Heuser and Reese, 1973) could reveal the possible link between synaptic vesicles and the vesicular chain. Recently, fluorescent compounds, called quantum dots, have been used in biological preparations to label cells via endocytosis. Quantum dots, unlike fluorescent molecules such as FM1-43, are resistant to photobleaching, have vary narrow excitation and emission spectra and cause very little damage to tissues during imaging. Quantum dots may be an ideal marker to label endocytosed vesicles to track their course through calyces and possibly determine if linkage between endocytosed vesicles and the vesicular chain exists.

\section{Maturation of MSO cells}

\section{Intrinsic properties}

Proper understanding of the physiological mechanisms underlying sound localization requires a detailed knowledge of the neurons of the auditory brainstem. We aimed to study the intrinsic and synaptic properties of binaural comparator neurons in the MSO by using electrophysiological techniques. Since whole cell recordings are easier to perform in early postnatal animals, we charted the early maturation MSO neurons. In chapter 3 we reported measurements of the electrophysiological changes that MSO cells undergo during the first few weeks of postnatal life. This study was undertaken to determine the time frame in which MSO neurons become more adult-like, in order to focus our studies that used simulated ITDs on more mature animals (Chapter 4). We concluded that the onset of hearing (PD12) is an important step in maturation of auditory brainstem neurons. The majority of MSO neurons after PD 12 exhibit a phasic firing pattern and have fast membrane time constants. We also found that equal amounts of hyperpolarizing and depolarizing current injections had non-linear effects on the membrane potential with rectification at more depolarized voltages. The phasic response of MSO cells is similar to those reported in bushy (Manis 
and Marx, 1991) and octopus cells (Golding et al., 1995) of the cochlear nucleus. Phasic responses are also found in the first order auditory nuclei of avians (Soares et al, 2002; Rathouz and Trussell, 1998) including nucleus laminaris, the homologue of the MSO (Reyes et al., 1994; Reyes et al., 1996). The phasic response is due in part to a $\alpha-D T X$ sensitive potassium conductance (Chapter 5). Our results were confirmed in a recent paper showing addition of $\alpha$-DTX into the bath eliminates phasic responses in gerbil MSO neurons (Svirskis et al., 2002). That the MSO cell matures to a phasic cell and has similar responses to other auditory nuclei suggest that phasic responses are important for mediating accurate transmission of temporal information (Studied in Chapter 5).

Our recordings indicate that the MSO principal cell column also contains a population of cells that respond tonically to current injection, even after the onset of hearing. A subpopulation of neurons within the tonic group had distinct afterhyperpolarizations with multiple components, which is typical of neurons that contain calcium activated potassium channels. The majority of tonic neurons did not have afterhyperpolarizations with multiple components, but had afterhyperpolarizations that were similar to those of phasic neurons. It is possible that the tonic neurons with afterhyperpolarizations that are similar to phasic neurons reflect a population of neurons that transition to phasic neurons later in life and the neurons with afterhyperpolarizations with multiple components remain throughout life.

\section{Inhibitory inputs}

In order to better understand the maturation of inhibitory inputs, we made recordings from MSO cells and stimulated one of their afferent inhibitory nuclei, the MNTB. We found that after the first week of life, stimulation of the MNTB produced a strychnine sensitive, hyperpolarizing IPSP. We made measurements of the time constant of recovery of IPSPs and found that IPSPs recovered more quickly to baseline after the onset of hearing. We concluded that the increased speed in recovery of IPSPs to baseline was mainly due to the concomitant decrease in the membrane time constant. 


\section{Suggestions}

Due to the difficulty in recording from the MSO beyond the second week of life we are unable to draw conclusions regarding the membrane electrical properties of MSO cells beyond PD 18. Overcoming the technical challenge of recording in older animals would be valuable in confirming that the phasic response is exhibited in adult neurons. One method may be to make intracellular recordings in a "blind" fashion with sharp electrodes in slices or in vivo animals.

Our results indicate that the ion channels that determine the response type most likely change throughout the first weeks of life. We did not, however, seek to identify the type(s) of potassium channels embedded in MSO cells. Immunocytochemical experiments could be performed to determine if the type, density, and number of voltage gated potassium channels coincide with our electrophysiological results. Studies that looked at expression levels of high threshold potassium channels (Kv3.1) in the cochlear nucleus homologue in embryonic chicks showed that there is an upregulation of potassium channels at about embryonic day 11 (Feng and Morest, 2001). Other experiments have showed that Kv3.1 transcripts increase in inferior colliculus neurons before hearing onset and then transcript levels remain constant (Liu and Kaczmarek, 2001). These anatomical experiments should be extended to look at the high threshold potassium channels (Kv3) and low threshold channels (Kv1.1) in neurons of the MSO and be coupled with voltage clamp experiments that isolate potassium currents. Our results also indicate dramatic change in the membrane time constant after the onset of hearing, which we hypothesized, are a result of

changes in potassium conductances. It is unclear if these changes occur as a result of activity driven input, and could be addressed by noting if such changes occur in deafened animals.

\section{Generation of simulated ITDs; characterization of the model cell}

We labeled cells in Study 2 and used one of those cells to construct a model MSO cell for generating stimuli to study ITD coding (Chapter 5). We chose a cell that had similarly weighted dendritic fields oriented towards each ear as estimated by their measured dendritic lengths. We characterized the 
passively modeled cell by injections of simulated excitatory current at various locations in the dendrites. We found that by moving the location of the simulated current injection more distally on the dendrite the time to peak of the EPSP in the soma was increased. Interestingly, the differences in the time to peak of the EPSP between the most proximal and the most distal locations could be as large as $800 \mu$ s (Chapter 4), far beyond the range of ITDs the gerbil can experience $(+/-120 \mu \mathrm{s})$. These results indicate that the placements of inputs onto each of the dendritic fields are critical in binaural computations. These results could possibly replace theories of axonal delay lines with synaptic placement delay lines, and merit further study.

There are many parameters that could be changed in future studies to further characterize MSO cells by using neuronal simulations. We chose to model a cell that had equally weighted dendritic fields, which was a cell within a distribution in which most cells were weighted to one side or the other. Measurements of MSO cell dendrites by other investigators indicate that the dendritic bias shifts from a slight lateral to slight medial emphasis between P18 and adult. Future studies should include models of cells with unequally weighted sides to determine the effects of unequal synaptic weights. Our finding that the lateral side of MSO cells had larger measured dendritic lengths might indicate that any attenuation in the level of the sound on the contralateral side may be compensated for by an increase in synaptic strength on the larger dendritic fields.

\section{The roles of voltage gated potassium channels and synaptic inhibition on the coding of ITDs by MSO cells.}

We used stimuli generated in Chapter 4 as current injection stimuli to study the coding of ITDs by MSO neurons. We concluded (see above) that potassium channels were the main contributor to the phasic response type and the sensitivity to ITD that we observed. Much work remains to determine the channels and currents that dictate MSO responses. By using immunocytochemistry against the suspected Kv1 family one could confirm and show their distribution along the cell body and dendrites. We suggest that fundamental differences in potassium channel type and/or distribution exist 
between phasic and tonic cells. Phasic cells most likely contain a high density of $\mathrm{Kv} 1$ channels along their dendritic trees where the effect on afferent input is the greatest. Tonic neurons probably lack these channels or contain separate families of potassium channels, such as calcium activated potassium channels. We were unable however, to completely rule out the possibility that sodium channel inactivation contributes to the phasic response type. We suggest that future experiments include voltage clamp studies to determine the profiles of sodium and potassium currents between phasic and tonic cells.

We identified, in a small population of tonic cells, distinct afterhyperpolarizations, that are consistent with studies of calcium activated potassium channels. Currently, there are no anatomical studies that screened neurons of the auditory brainstem for these types of channels. Immunocytochemistry experiments using antibodies directed against calcium activated potassium channels would reveal the subpopulation of MSO neurons that may express these channels. Neurons that exhibit calcium-activated potassium channel like afterhyperpolarizations should be tested with protocols that contain very long (2 to 10 seconds) depolarizing current pulses. Repetitive action potentials should increase the calcium concentration within the cell and further activate calcium activated potassium channels, thereby decreasing the input resistance of the cell. At some time point, the action potentials would cease, indicating a possible potassium conductance.

By our measure, tonic neurons were only slightly sensitive to the change in ITD. These neurons may reflect the pool of neurons recorded from MSO in vivo of several species that are insensitive to ITDs (gerbil: Brand et al. 2002; dog: Goldberg and Brown 1969; cat: Yin and Chan 1990). Since the tonic cells increased their firing rate with increasing current injection, those cells may contribute to the coding of stimulus amplitude rather than coding for ITDs.

We generated simulated excitatory postsynaptic potentials in the model cell to use as stimuli in the in vitro experiments. The EPSPs were approximately $10 \mathrm{~ms}$ in duration, which is within the range of recorded EPSPs (Smith, 1993) but near the reported upper limit. We chose to stimulate MSO cells with long 
simulated EPSPs because our simulations indicated more robust interactions when EPSPs were longer than IPSPs. Future studies should shorten the time of the alpha function, thereby shortening the EPSPs used in the in vitro experiments. Shortening of EPSPs would be expected if the temperature of the preparation were raised to body temperature $\left(38^{\circ} \mathrm{C}\right)$ rather than the experimental temperature $\left(30^{\circ} \mathrm{C}\right)$. Similar results are expected with shorter EPSPs to those obtained with longer EPSPs, except ITD curves may be steeper due to the decrease in the total amount of current injected with shorter EPSPs. We used different gains (amplitudes) of simulated EPSP current injections to test the gain modulating ability that the cell possesses. Interestingly, we found that by increasing the gain of the simulated EPSP current injections (increasing corner ITD), MSO cells had similar ITD sensitivities regardless of the gain used (for only those that entrained stimuli). Loud sounds increase the amount of synaptic excitation that the cell receives (our large amplitude stimuli). The continued sensitivity may be the result of the increased potassium conductance in the depolarized range. As synaptic excitation increases there is a concomitant increase in the potassium current, decreasing the effectiveness of the large EPSPs. Experiments that use similar stimuli should be performed in the voltage clamp mode to measure the size of potassium currents at different synaptic excitation levels.

We demonstrated for the first time in living neurons that the timing of inhibitory synaptic inputs relative to excitatory inputs significantly shapes ITD curves. We tested the influence of inhibitory neurotransmission on the coding of ITDs by MSO by stimulation of only one of the inhibitory nuclei projecting to the MSO. Future experiments should also stimulate the ipsilaterally activated cell group called the LNTB. The relative timing of inhibitory inputs from the LNTB compared to the MNTB and excitatory inputs are unknown. Knowledge of the timing of inhibitory inputs is essential in determining the output of MSO neurons to determine the sound source locations where leading, simultaneous or lagging inhibition predominates.

Dependent upon the arrival time of IPSPs, sensitivity to ITDs could be 
elicited, where in control situations there were none (lagging). In other situations a complete suppression of action potentials was observed (simultaneous) or suppression of action potentials at short ITDs, followed by an increase in spike counts at longer ITDs ("dome" response; leading ITDs).

The response ITD curves that were generated can be explained by examining the integration of excitatory inputs with inhibitory inputs. We used NEURON simulations to investigate how how inhibitory inputs interact with excitatory inputs to give a qualitative explanation of our results (Figure 1), which are described below. The simulation was performed on the same model cell used in the previous experiments, but with added inhibitory inputs (reversal voltage $=-80$ $\mathrm{mV}$ ) placed on the cell body. For simplicity, the simulations were run at only two ITDs (0 and $0.5 \mathrm{~ms})$.

In our in vitro experiments, when IPSPs arrived after the onset of the EPSPs (lagging), responses were shifted from longer corner ITDs to shorter ones. The NEURON simulation shows that lagging inhibition significantly suppresses the response at long ITDs (Figure 1A) below action potential threshold (horizontal line). This had the effect of eliminating action potential firing at long ITDs and only those excitatory inputs arriving at or very near coincidence sum to action potential threshold. The simulations describe our results in situations where activation of inhibitory inputs shifted corner ITDs to shorter ITD values.

In our in vitro experiments, when inhibitory inputs were activated at or very near the start of the EPSP, we found the most dramatic decrease in the number of action potentials generated. The EPSPs of the model cell were dramatically reduced by simultaneous inhibition and decreased the resultant EPSPs below action potential threshold at both ITDs (Figure 1B). In simultaneous inhibition conditions, most of the IPSP overlaps with the EPSP and reduces the depolarization to values below threshold.

Our most intriguing result from the in vitro experiments was that when inhibitory inputs lead the EPSP current injection, ITD curves could be "dome" shaped in appearance. The results from the NEURON simulation may explain 
this result. When the IPSP preceded the EPSP, the membrane hyperpolarized and decreased the amplitude of the EPSP at zero ITD to a value below action potential threshold. At $0.5 \mathrm{~ms}$ ITD, the EPSP peaked during the recovery phase of the IPSP, and the resultant EPSP was of slightly higher amplitude than occurred at zero ITD. The increase caused the EPSP to reach action potential threshold (Figure 1C). At longer ITDs (not shown) the amplitudes of the EPSPs decreased below action potential threshold.

Our results indicate that at certain inhibitory delays the activity of MSO cells can be greatly enhanced or suppressed. What is the purpose of having a system where delicate changes in inhibitory timing effect spike generation? We propose the following model (see Figure 2 to correspond with the following description). The relative timing of excitation and inhibition from a single ear remains constant, but since MNTB inputs are contralaterally activated they could either precede or follow excitation (explained below). If a sound is coming from directly in front of the head, the sound reaches both ears at the same time (zero ITD). Due to the fast conduction velocity of globular bushy cell axons, (see Chapter 1) inhibition could slightly precede excitation at neurons $A$ and $B$ and suppress action potentials in both neurons to $50 \%$ of maximum level. This situation is similar to the $0 \mathrm{~ms}$ ITD modeled in Figure $1 \mathrm{C}$. If the sound moves to the right, (toward neuron $B$ ) thus generating a non-zero ITD, the relative timing of inhibition, compared to zero ITD, in each neuron is now different. Since the sound entered the right ear first, and the inhibition is faster than the excitation, the first input to neuron $A$ is inhibitory. Due to the ITD associated with the movement of the sound source, neuron A generates action potentials above the $50 \%$ level at zero ITD. This situation is similar to the $0.5 \mathrm{~ms}$ ITD modeled in Figure 1C. We have shown in our in vitro experiments, that with leading inhibitory events action potential generation can be greater at longer ITDs than at zero ITD (our "dome" response).

Since the sound moved to the right, the first input to neuron $B$ is excitation from the ipsilateral cochlear nucleus. The activation of the ipsilateral MNTB is delayed by the ITD, and therefore the inhibitory input to neuron $B$ is delayed by 
the same amount. Compared to 0 ITD, the inhibition is pushed closer to the start of the excitatory synaptic input and decreases the spike rate. In this situation, the contralateral inhibition could begin simultaneously with the ipsilateral excitation, which is similar to the $0.5 \mathrm{~ms}$ ITD modeled in Figure 1B. In our experiments, inhibition at or near the start of EPSPs drastically reduced action potential generation (our simultaneous onset responses).

In this model, movement of the sound source from the midline to the right (side B in Figure 2) causes activity in the MSO contralateral to the sound source (side A in Figure 2) to increase whereas the ipsilateral MSO shows decreased activity. These results are consistent with those from in vivo data indicating that MSO cells respond to contralateral stimuli (Yin and Chan, 1990).

Overall, our results from the in vitro and modeling experiments indicate that membranes of MSO cells have evolved specialized potassium conductances suited to detect small changes in temporal stimuli. Also, slight changes in the relative timing of excitatory and inhibitory input are critical factors that determine the outflow of MSO neurons. These two specializations work in concert to encode the location of sound sources to higher brain centers. 


\section{A. Lagging inhibition}

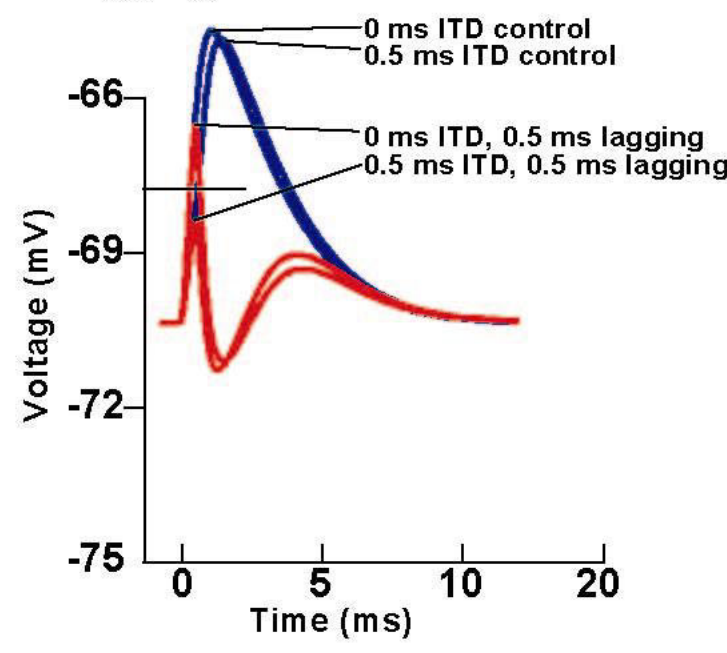

\section{B. Simultaneous inhibition}

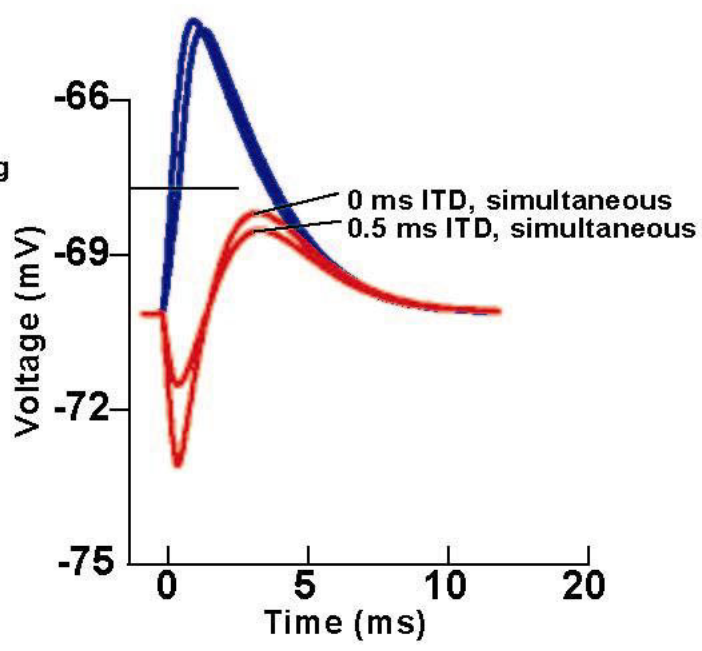

\section{Leading inhibition}

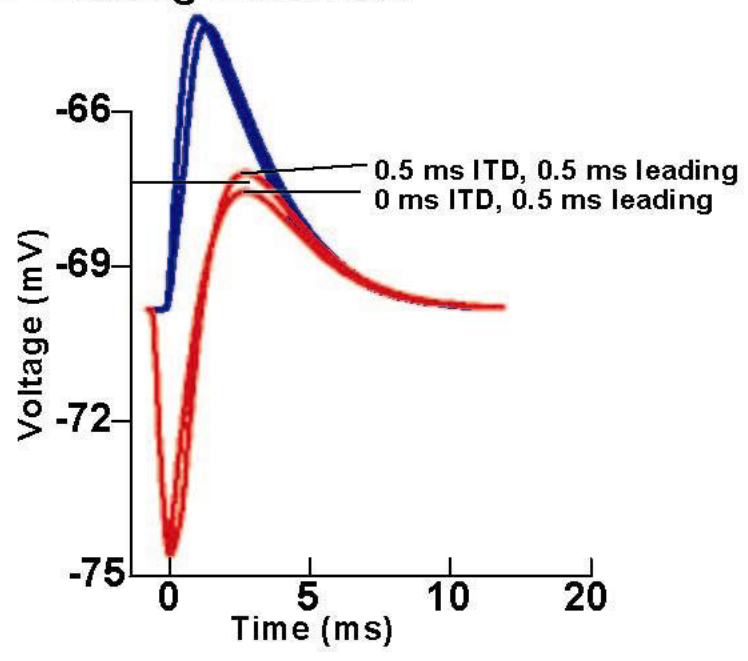

Figure 1. Integration of excitatory and inhibitory synaptic input in a model cell.

A. Lagging inhibition caused a decrease in the amplitudes of the postsynaptic responses (red curves) compared to control (blue curves; excitatory input alone). At a longer ITD $(0.5 \mathrm{~ms})$ inhibition caused the postsynaptic response to decline even further than 0 ITD. B: Simultaneous inhibition caused a large decline at 0 
ITD and still further decreases at longer ITDs. C: Leading inhibition caused a greater decline at 0 ITD than at slightly longer ITDs. Horizontal line represents proposed action potential threshold. 


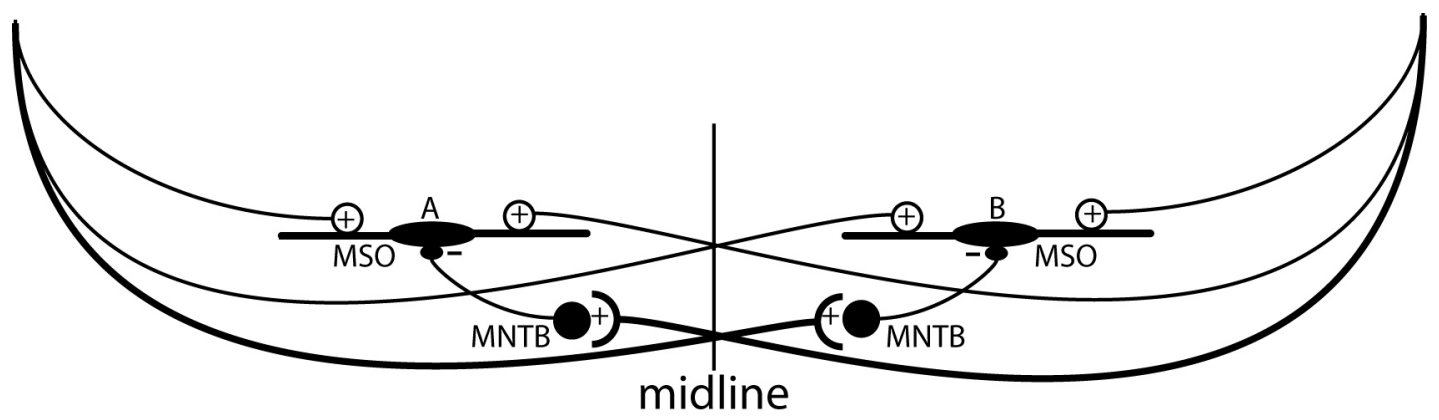

Figure 2. Schematic of proposed MSO model.

$A$ and $B$ represent single neurons of the MSO. Plus symbols represent excitatory input from bushy cells of the cochlear nucleus. Minus symbols represent inhibitory input from MNTB cells. 
Curriculum Vitae 
Kevin C. Rowland

(Updated: May 9, 2003)

Business Address: Department of Physiology and Pharmacology

Box 9229, Robert C. Byrd Health Sciences Center

West Virginia University School of Medicine

Morgantown, WV 26505-9229

Phone: (304) 293-1308

e-mail: krowland@wvu.edu

Professional Position

1996-Present Graduate Research Assistant

Department of Physiology

Education

West Virginia University, Morgantown, WV

West Virginia University B.A. Biology

West Virginia University Ph.D. Physiology

(Ph.D. candidate 10/99)

Research Interests

Neural mechanisms underlying sound localization

Computer modeling of neurons

Teaching Experience

West Virginia University

Physiology 441 (Human Physiology)

200 undergraduate nursing and allied health students

2001

Lecturer, Topic: action potentials, synaptic transmission

Physiology 241 (Human Physiology)

200 undergraduate nursing and allied health students

2000

Lecturer, Topic: Synaptic transmission

Human Function

Small group facilitator for 12 medical students

1998

Honors and Awards

First Annual Sigma Xi WVU Graduate Student Competition: $2^{\text {nd }}$ place. 2000

This competition is open to all University students.

West Virginia Neuroscience Chapter Graduate Student Competition: $1^{\text {st }}$ place.

1998

This competition is open to all students enrolled in the Neuroscience Graduate Program.

Julie Betschart Memorial Graduate Student Competition: $1^{\text {st }}$ place.

1997

This competition is open to all students enrolled in the Department of Physiology.

These competitions are based on oral/poster presentation of current research.

\section{Publications}

Manuscripts

Taschenberger H, Leão R, Rowland KC, Spirou GA, and von Gersdorff H. Optimizing synaptic ultrastructure, functional capacity and efficiency for prolonged high frequency firing. Neuron 2002 Dec 19; 36(6):1127-43

Rowland, KC, Irby NK, and Spirou GA. Specialized synapse-associated structures within the Calyx of Held. J. Neurosci. 2000 Dec 15; 20(24):9135-44. 
Spirou, GA, Rowland, KC and Berrebi, AS. Ultrastructure of neurons and large synaptic terminals in the lateral nucleus of the trapezoid body of the cat. J. Comp Neurol. 1998 Aug 24;398(2):257-72

\author{
Abstracts \\ Rowland KC, Spirou GA. Interactions of excitatory and inhibitory synaptic inputs in the MSO. \\ ARO, 2002.
}

Rowland KC, Spirou GA. Maturation of membrane electrical properties in gerbil medial superior olive. Society for Neuroscience, 2001

Rowland KC, Spirou GA. Mitochondria-associated adherens complexes in calyces of Held are located adjacent to synapses. ARO, 2000

Rowland KC, Spirou GA. The adherens organelle, a specialized structure of large nerve terminals in the auditory brainstem. ARO, 1998

Spirou GA, Rowland KC, Berrebi AS. Ultrastructural features of neurons in the cat lateral nucleus of the trapezoid body. ARO, 1997. 


\section{References}

1. Adams, J. C. Ascending projections to the inferior colliculus. J.Comp Neurol. 183: 519-538, 1979.

2. Adams, J. C. and Mugnaini, E. Immunocytochemical evidence for inhibitory and disinhibitory circuits in the superior olive. Hear.Res. 49: 281-298, 1990.

3. Babcock, D. F., Herrington, J., Goodwin, P. C., Park, Y. B., and Hille, B. Mitochondrial participation in the intracellular Ca2+ network. J.Cell Biol. 136: 833844, 1997.

4. Bal, R. and Oertel, D. Potassium currents in octopus cells of the mammalian cochlear nucleus. J.Neurophysiol. 86: 2299-2311, 2001.

5. Banks, M. I. and Sachs, M. B. Regularity analysis in a compartmental model of chopper units in the anteroventral cochlear nucleus. J Neurophysiol. 65: 606-629, 1991.

6. Banks, M. I. and Smith, P. H. Intracellular recordings from neurobiotin-labeled cells in brain slices of the rat medial nucleus of the trapezoid body. J Neurosci. 12: 2819-2837, 1992.

7. Bellingham, M. C. and Walmsley, B. A novel presynaptic inhibitory mechanism underlies paired pulse depression at a fast central synapse. Neuron 23: 159-170, 1999.

8. Berrebi, A. S. and Mugnaini, E. Distribution and targets of the cartwheel cell axon in the dorsal cochlear nucleus of the guinea pig. Anat.Embryol.(Berl) 183: 427454, 1991. 
9. Berrebi, A. S. and Spirou, G. A. PEP-19 immunoreactivity in the cochlear nucleus and superior olive of the cat. Neuroscience 83: 535-554, 1998.

10. Beyerl, B. D. Afferent projections to the central nucleus of the inferior colliculus in the rat. Brain Res. 145: 209-223, 1978.

11. Bhattacharjee, A., Gan, L., and Kaczmarek, L. K. Localization of the Slack potassium channel in the rat central nervous system. J Comp Neurol. 454: 241$254,2002$.

12. Bodian, D. Electron microscopy: two major synaptic types on spinal motoneurons. Science 151: 1093-1094, 1966.

13. Borst, J. G., Helmchen, F., and Sakmann, B. Pre- and postsynaptic whole-cell recordings in the medial nucleus of the trapezoid body of the rat. J.Physiol 489 ( Pt 3): 825-840, 1995.

14. Borst, J. G. and Sakmann, B. Depletion of calcium in the synaptic cleft of a calyxtype synapse in the rat brainstem. J.Physiol $521 \mathrm{Pt}$ 1: 123-133, 1999a.

15. Borst, J. G. and Sakmann, B. Effect of changes in action potential shape on calcium currents and transmitter release in a calyx-type synapse of the rat auditory brainstem. Philos.Trans.R.Soc.Lond B Biol.Sci. 354: 347-355, 1999 b.

16. Boudreau, J. C. and Tsuchitani, C. Binaural interaction in the cat superior olive $S$ segment. J Neurophysiol. 31: 442-454, 1968.

17. Brand, A., Behrend, O., Marquardt, T., McAlpine, D., and Grothe, B. Precise inhibition is essential for microsecond interaural time difference coding. Nature 417: 543-547, 2002. 
18. Brew, H. M. and Forsythe, I. D. Two voltage-dependent $\mathrm{K}+$ conductances with complementary functions in postsynaptic integration at a central auditory synapse. J.Neurosci. 15: 8011-8022, 1995.

19. Brose, N. Synaptic cell adhesion proteins and synaptogenesis in the mammalian central nervous system. Naturwissenschaften 86: 516-524, 1999.

20. Brunso-Bechtold, J. K., Thompson, G. C., and Masterton, R. B. HRP study of the organization of auditory afferents ascending to central nucleus of inferior colliculus in cat. J.Comp Neurol. 197: 705-722, 1981.

21. Cai, Y., McGee, J., and Walsh, E. J. Contributions of ion conductances to the onset responses of octopus cells in the ventral cochlear nucleus: simulation results. J.Neurophysiol. 83: 301-314, 2000.

22. Cajal, R 1909.

23. Cangiano, L., Wallen, P., and Grillner, S. Role of apamin-sensitive k(ca) channels for reticulospinal synaptic transmission to motoneuron and for the afterhyperpolarization. J Neurophysiol. 88: 289-299, 2002.

24. Cant, N. B. and Casseday, J. H. Projections from the anteroventral cochlear nucleus to the lateral and medial superior olivary nuclei. J.Comp Neurol. 247: 457-476, 1986.

25. Cant, N. B. and Hyson, R. L. Projections from the lateral nucleus of the trapezoid body to the medial superior olivary nucleus in the gerbil. Hear.Res. 58: 26-34, 1992a.

26. Cant, N. B. and Hyson, R. L. Projections from the lateral nucleus of the trapezoid body to the medial superior olivary nucleus in the gerbil. Hear.Res. 58: 26-34, 1992b. 
27. Cant, N. B. and Morest, D. K. The bushy cells in the anteroventral cochlear nucleus of the cat. A study with the electron microscope. Neuroscience 4: 19251945, 1979.

28. Casey, M. A. and Feldman, M. L. Aging in the rat medial nucleus of the trapezoid body. II. Electron microscopy. J.Comp Neurol. 232: 401-413, 1985.

29. Casseday, J. H. and Neff, W. D. Auditory localization: role of auditory pathways in brain stem of the cat. J.Neurophysiol. 38: 842-858, 1975.

30. Ceccarelli, B., Hurlbut, W. P., and Mauro, A. Turnover of transmitter and synaptic vesicles at the frog neuromuscular junction. J.Cell Biol. 57: 499-524, 1973.

31. Clark, G. M. The ultrastructure of nerve endings in the medial superior olive of the cat. Brain Res. 14: 293-305, 1969a.

32. Clark, G. M. Vesicle shape versus type of synapse in the nerve endings of the cat medial superior olive. Brain Res. 15: 548-551, $1969 \mathrm{~b}$.

33. Colonnier, M. and Guillery, R. W. Synaptic organization in the lateral geniculate nucleus of the monkey. Z.Zellforsch.Mikrosk.Anat. 62: 333-355, 1964.

34. David, G., Barrett, J. N., and Barrett, E. F. Evidence that mitochondria buffer physiological Ca2+ loads in lizard motor nerve terminals. J.Physiol 509 ( Pt 1): 59-65, 1998.

35. Denton, R. M. and McCormack, J. G. The role of calcium in the regulation of mitochondrial metabolism. Biochem.Soc.Trans. 8: 266-268, 1980.

36. Dodson, P. D., Barker, M. C., and Forsythe, I. D. Two heteromeric Kv1 potassium channels differentially regulate action potential firing. J.Neurosci. 22: 6953-6961, 2002a. 
37. Dodson, P. D., Barker, M. C., and Forsythe, I. D. Two heteromeric Kv1 potassium channels differentially regulate action potential firing. J Neurosci. 22: 6953-6961, 2002b.

38. Elverland, H. H. Descending connections between superior olivary and cochlear nuclear complexes in the cat studied by autoradiographic and horseradish peroxidase methods. Exp.Brain Res. 27: 397-12, 1977.

39. Fekete, D. M., Rouiller, E. M., Liberman, M. C., and Ryugo, D. K. The central projections of intracellularly labeled auditory nerve fibers in cats. J.Comp Neurol. 229: 432-450, 1984.

40. Ferragamo, M. J. and Oertel, D. Octopus cells of the mammalian ventral cochlear nucleus sense the rate of depolarization. J.Neurophysiol. 87: 2262-2270, 2002.

41. Ferroni, S., Planells-Cases, R., Ahmed, C. M., and Montal, M. Expression of a genomic clone encoding a brain potassium channel in mammalian cells using lipofection. Eur.Biophys.J 21: 185-191, 1992.

42. Forsythe, I. D. and Barnes-Davies, M. The binaural auditory pathway: excitatory amino acid receptors mediate dual timecourse excitatory postsynaptic currents in the rat medial nucleus of the trapezoid body. Proc.R.Soc.Lond B Biol.Sci. 251: 151-157, 1993a.

43. Forsythe, I. D. and Barnes-Davies, M. The binaural auditory pathway: membrane currents limiting multiple action potential generation in the rat medial nucleus of the trapezoid body. Proc.R.Soc.Lond B Biol.Sci. 251: 143-150, 1993 b.

44. Forsythe, I. D., Tsujimoto, T., Barnes-Davies, M., Cuttle, M. F., and Takahashi, T. Inactivation of presynaptic calcium current contributes to synaptic depression at a fast central synapse. Neuron 20: 797-807, 1998. 
45. Furst, M. and Algom, D. Lateralization and discrimination of dichotic clicks: evidence from patients with brainstem lesions and normal cohorts. J Basic Clin.Physiol Pharmacol. 6: 149-171, 1995.

46. Gentschev, T. and Sotelo, C. Degenerative patterns in the ventral cochlear nucleus of the rat after primary deafferentation. An ultra-structural study. Brain Res. 62: 37-60, 1973.

47. Glendenning, K. K. and Baker, B. N. Neuroanatomical distribution of receptors for three potential inhibitory neurotransmitters in the brainstem auditory nuclei of the cat. J.Comp Neurol. 275: 288-308, 1988.

48. Glendenning, K. K., Brunso-Bechtold, J. K., Thompson, G. C., and Masterton, R. B. Ascending auditory afferents to the nuclei of the lateral lemniscus. J.Comp Neurol. 197: 673-703, 1981.

49. Glendenning, K. K. and Masterton, R. B. Acoustic chiasm: efferent projections of the lateral superior olive. J.Neurosci. 3: 1521-1537, 1983.

50. Goldberg, J. M. and Brown, P. B. Functional organization of the dog superior olivary complex: an anatomical and electrophysiological study. J.Neurophysiol. 31: 639-656, 1968.

51. Goldberg, J. M. and Brown, P. B. Response of binaural neurons of dog superior olivary complex to dichotic tonal stimuli: some physiological mechanisms of sound localization. J Neurophysiol. 32: 613-636, 1969.

52. Golding, N. L., Ferragamo, M. J., and Oertel, D. Role of intrinsic conductances underlying responses to transients in octopus cells of the cochlear nucleus. J.Neurosci. 19: 2897-2905, 1999. 
53. Golowasch, J., Buchholtz, F., Epstein, I. R., and Marder, E. Contribution of individual ionic currents to activity of a model stomatogastric ganglion neuron. $J$ Neurophysiol. 67: 341-349, 1992.

54. Gray, E. Electron microscopy of presynaptic organelles of the spinal cord. J Anat Lond 97: 101-106, 1963.

55. Grigg, J. J., Brew, H. M., and Tempel, B. L. Differential expression of voltagegated potassium channel genes in auditory nuclei of the mouse brainstem. Hear.Res. 140: 77-90, 2000.

56. Grissmer, S., Nguyen, A. N., Aiyar, J., Hanson, D. C., Mather, R. J., Gutman, G. A., Karmilowicz, M. J., Auperin, D. D., and Chandy, K. G. Pharmacological characterization of five cloned voltage-gated $\mathrm{K}+$ channels, types $\mathrm{Kv1} 1.1,1.2,1.3$, 1.5, and 3.1, stably expressed in mammalian cell lines. Mol.Pharmacol. 45: 12271234, 1994.

57. Grothe, B. and Sanes, D. H. Bilateral inhibition by glycinergic afferents in the medial superior olive. J.Neurophysiol. 69: 1192-1196, 1993.

58. Guinan, J. J., Jr. and Li, R. Y. Signal processing in brainstem auditory neurons which receive giant endings (calyces of Held) in the medial nucleus of the trapezoid body of the cat. Hear.Res. 49: 321-334, 1990.

59. Gulley, R. L., Landis, D. M., and Reese, T. S. Internal organization of membranes at end bulbs of Held in the anteroventral cochlear nucleus. J.Comp Neurol. 180: 707-741, 1978.

60. Harris, K. M. and Sultan, P. Variation in the number, location and size of synaptic vesicles provides an anatomical basis for the nonuniform probability of release at hippocampal CA1 synapses. Neuropharmacology 34: 1387-1395, 1995. 
61. Harvey, A. L. Twenty years of dendrotoxins. Toxicon 39: 15-26, 2001.

62. Harvey, A. L. and Karlsson, E. Dendrotoxin from the venom of the green mamba, Dendroaspis angusticeps. A neurotoxin that enhances acetylcholine release at neuromuscular junction. Naunyn Schmiedebergs Arch.Pharmacol. 312: 1-6, 1980.

63. Harvey, A. L. and Karlsson, E. Protease inhibitor homologues from mamba venoms: facilitation of acetylcholine release and interactions with prejunctional blocking toxins. Br.J Pharmacol. 77: 153-161, 1982.

64. Held, H. Die centrale Gehorleitung. Archiv fur Anatomie und Physiologie.Anat Abt 201-248, 1893.

65. Helfert, R. H., Bonneau, J. M., Wenthold, R. J., and Altschuler, R. A. GABA and glycine immunoreactivity in the guinea pig superior olivary complex. Brain Res. 501: 269-286, 1989.

66. Henkel, C. K. and Brunso-Bechtold, J. K. Development of glycinergic cells and puncta in nuclei of the superior olivary complex of the postnatal ferret. J.Comp Neurol. 354: 470-480, 1995.

67. Henkel, C. K. and Spangler, K. M. Organization of the efferent projections of the medial superior olivary nucleus in the cat as revealed by HRP and autoradiographic tracing methods. J.Comp Neurol. 221: 416-428, 1983.

68. Heuser, J. E. and Reese, T. S. Evidence for recycling of synaptic vesicle membrane during transmitter release at the frog neuromuscular junction. J.Cell Biol. 57: 315-344, 1973.

69. Hille, B. G protein-coupled mechanisms and nervous signaling. Neuron 9: 187195, 1992. 
70. Hodgkin, A., Huxley, A., and Katz, B. Ionic currents underlying activity in the giant axon of the squid. Archives des Sciences Physiologiques 3: 129-150, 1949.

71. Jack, J. J. Electric current flow in excitable cells. Oxford, Clarendon Press. 1983.

72. Jan, L. and Jan, Y. Voltage-gated and inwardly rectifying potassium channels. J.Physiol 505: 267-282, 1997.

73. Jean-Baptiste, M. and Morest, D. Transneuronal changes of synaptic endings and nuclear chromatin in the trapezoid body following cochlear ablation in cats. J.Comp Neurol. 162: 111-134, 1975.

74. Jeffress, L. A. A Place Theory of Sound Localization. Journal of Comparative Physiology and Psychology 41: 35-39, 1948.

75. Jenkins, W. M. and Masterton, R. B. Sound localization: effects of unilateral lesions in central auditory system. J.Neurophysiol. 47: 987-1016, 1982.

76. Jonas, E. A., Buchanan, J., and Kaczmarek, L. K. Prolonged activation of mitochondrial conductances during synaptic transmission. Science 286: 13471350, 1999.

77. Jones, D. G. and Cullen, A. M. A quantitative investigation of some presynaptic terminal parameters during synaptogenesis. Exp.Neurol. 64: 245-259, 1979.

78. Joris, P. X., Carney, L. H., Smith, P. H., and Yin, T. C. Enhancement of neural synchronization in the anteroventral cochlear nucleus. I. Responses to tones at the characteristic frequency. J.Neurophysiol. 71: 1022-1036, 1994a.

79. Joris, P. X., Smith, P. H., and Yin, T. C. Enhancement of neural synchronization in the anteroventral cochlear nucleus. II. Responses in the tuning curve tail. J.Neurophysiol. 71: 1037-1051, 1994b. 
80. Kaftan, E. J., Xu, T., Abercrombie, R. F., and Hille, B. Mitochondria shape hormonally induced cytoplasmic calcium oscillations and modulate exocytosis. J.Biol.Chem. 275: 25465-25470, 2000.

81. Kapfer, C., Seidl, A. H., Schweizer, H., and Grothe, B. Experience-dependent refinement of inhibitory inputs to auditory coincidence-detector neurons. Nat.Neurosci. 5: 247-253, 2002.

82. Kimura, R. S., Bongiorno, C. L., and Iverson, N. A. Synapses and ephapses in the spiral ganglion. Acta Otolaryngol.Suppl 438: 1-18, 1987.

83. Kiss, A. and Majorossy, K. Neuron morphology and synaptic architecture in the medial superior olivary nucleus. Light- and electron microscope studies in the cat. Exp.Brain Res. 52: 315-327, 1983.

84. Kohmura, N., Senzaki, K., Hamada, S., Kai, N., Yasuda, R., Watanabe, M., Ishii, H., Yasuda, M., Mishina, M., and Yagi, T. Diversity revealed by a novel family of cadherins expressed in neurons at a synaptic complex. Neuron 20: 1137-1151, 1998.

85. Kudo, M., Nakamura, Y., Tokuno, H., and Kitao, Y. Auditory brainstem in the mole (Mogera): nuclear configurations and the projections to the inferior colliculus. J.Comp Neurol. 298: 400-412, 1990.

86. Kuromi, H. and Kidokoro, Y. The optically determined size of exo/endo cycling vesicle pool correlates with the quantal content at the neuromuscular junction of Drosophila larvae. J.Neurosci. 19: 1557-1565, 1999.

87. Kuwabara, N., DiCaprio, R. A., and Zook, J. M. Afferents to the medial nucleus of the trapezoid body and their collateral projections. J.Comp Neurol. 314: 684-706, 1991. 
88. Kuwabara, N. and Zook, J. M. Projections to the medial superior olive from the medial and lateral nuclei of the trapezoid body in rodents and bats. J.Comp Neurol. 324: 522-538, 1992.

89. Lenn, N. J. and Reese, T. S. The fine structure of nerve endings in the nucleus of the trapezoid body and the ventral cochlear nucleus. Am.J Anat 118: 375-389, 1966.

90. Lieberman, A. R. and Spacek, J. Filamentous contacts: the ultrastructure and three-dimensional organization of specialized non-synaptic interneuronal appositions in thalamic relay nuclei. Cell Tissue Res. 288: 43-57, 1997.

91. Lindsey, B. G. Fine structure and distribution of axon terminals from the cochlear nucleus on neurons in the medial superior olivary nucleus of the cat. J.Comp Neurol. 160: 81-103, 1975b.

92. Lindsey, B. G. Fine structure and distribution of axon terminals from the cochlear nucleus on neurons in the medial superior olivary nucleus of the cat. J Comp Neurol. 160: 81-103, 1975a.

93. Ma, M. and Koester, J. The role of $\mathrm{K}+$ currents in frequency-dependent spike broadening in Aplysia R20 neurons: a dynamic-clamp analysis. J Neurosci. 16: 4089-4101, 1996.

94. Maechler, P. and Wollheim, C. B. Mitochondrial glutamate acts as a messenger in glucose-induced insulin exocytosis. Nature 402: 685-689, 1999.

95. Manis, P. B. and Marx, S. O. Outward currents in isolated ventral cochlear nucleus neurons. J.Neurosci. 11: 2865-2880, 1991.

96. Masterton, R. B. and Imig, T. J. Neural mechanisms for sound localization. Annu.Rev.Physiol 46: 275-287, 1984. 
97. Maycox, P. R., Deckwerth, T., Hell, J. W., and Jahn, R. Glutamate uptake by brain synaptic vesicles. Energy dependence of transport and functional reconstitution in proteoliposomes. J.Biol.Chem. 263: 15423-15428, 1988.

98. McAlpine, D., Jiang, D., and Palmer, A. R. A neural code for low-frequency sound localization in mammals. Nat.Neurosci. 4: 396-401, 2001.

99. Moore, J. K. Organization of the human superior olivary complex. Microsc.Res.Tech. 51: 403-412, 2000.

100. Moore, M. J. and Caspary, D. M. Strychnine blocks binaural inhibition in lateral superior olivary neurons. J.Neurosci. 3: 237-242, 1983.

101. Morest, D. K. The collateral system of the medial nucleus of the trapezoid body of the cat, its neuronal architecture and relation to the olivo-cochlear bundle. Brain Res. 9: 288-311, 1968.

102. Morest, D. K. Auditory neurons of the brain stem. Adv.Otorhinolaryngol. 20: 337356, 1973.

103. Moushegian, G., Rupert, A., and Whitcomb, M. Medial superior-olivary unit response patterns to monaural and binaural clicks. J Acoust.Soc.Am. 36: 196202, 1964.

104. Murthy, V. N. and Stevens, C. F. Synaptic vesicles retain their identity through the endocytic cycle. Nature 392: 497-501, 1998.

105. Nakajima, Y. Fine structure of the medial nucleus of the trapezoid body of the bat with special reference to two types of synaptic endings. J.Cell Biol. 50: 121-134, 1971. 
106. Neher, E. Vesicle pools and Ca2+ microdomains: new tools for understanding their roles in neurotransmitter release. Neuron 20: 389-399, 1998.

107. Neises, G. R., Mattox, D. E., and Gulley, R. L. The maturation of the end bulb of Held in the rat anteroventral cochlear nucleus. Anat Rec. 204: 271-279, 1982.

108. Nicol, M. J. and Walmsley, B. Ultrastructural basis of synaptic transmission between endbulbs of Held and bushy cells in the rat cochlear nucleus. J Physiol 539: 713-723, 2002.

109. Nordeen, K. W., Killackey, H. P., and Kitzes, L. M. Ascending auditory projections to the inferior colliculus in the adult gerbil, Meriones unguiculatus. J.Comp Neurol. 214: 131-143, 1983.

110. Oertel, D. Synaptic responses and electrical properties of cells in brain slices of the mouse anteroventral cochlear nucleus. J Neurosci. 3: 2043-2053, 1983.

111. Okoyama, S., Moriizumi, T., Kitao, Y., Kawano, J., and Kudo, M. Anatomical plasticity in the medial superior olive following ablation of the inferior colliculus in neonatal and adult rats. Hear.Res. 88: 71-78, 1995.

112. Oliver, D. L., Beckius, G. E., and Shneiderman, A. Axonal projections from the lateral and medial superior olive to the inferior colliculus of the cat: a study using electron microscopic autoradiography. J.Comp Neurol. 360: 17-32, 1995.

113. Osen, K. K. Cytoarchitecture of the cochlear nuclei in the cat. J.Comp Neurol. 136: 453-484, 1969.

114. Ostapoff, E. M., Benson, C. G., and Saint Marie, R. L. J Comp Neurol. 381: 500512, 1997. 
115. Parameshwaran, S., Carr, C. E., and Perney, T. M. Expression of the Kv3.1 potassium channel in the avian auditory brainstem. J Neurosci. 21: 485-494, 2001.

116. Perkins, G., Renken, C., Martone, M. E., Young, S. J., Ellisman, M., and Frey, T. Electron tomography of neuronal mitochondria: three-dimensional structure and organization of cristae and membrane contacts. J.Struct.Biol. 119: 260-272, 1997.

117. Perkins, R. E. An electron microscopic study of synaptic organization in the medial superior olive of normal and experimental chinchillas. J.Comp Neurol. 148: 387-415, 1973c.

118. Perkins, R. E. An electron microscopic study of synaptic organization in the medial superior olive of normal and experimental chinchillas. J Comp Neurol. 148: 387-415, 1973b.

119. Perkins, R. E. An electron microscopic study of synaptic organization in the medial superior olive of normal and experimental chinchillas. J Comp Neurol. 148: 387-415, 1973a.

120. Perney, T. M. and Kaczmarek, L. K. Localization of a high threshold potassium channel in the rat cochlear nucleus. J Comp Neurol. 386: 178-202, 1997.

121. Petelina, E. V. [Ultrastructure of synapses in the nucleus of the trapezoid body of the bat]. Arkh.Anat.Gistol.Embriol. 68: 14-18, 1975.

122. Peyret, D., Campistron, G., Geffard, M., and Aran, J. M. Glycine immunoreactivity in the brainstem auditory and vestibular nuclei of the guinea pig. Acta Otolaryngol. 104: 71-76, 1987. 
123. Pivovarova, N. B., Hongpaisan, J., Andrews, S. B., and Friel, D. D. Depolarization-induced mitochondrial $\mathrm{Ca}$ accumulation in sympathetic neurons: spatial and temporal characteristics. J.Neurosci. 19: 6372-6384, 1999.

124. Pratt, H., Polyakov, A., Aharonson, V., Korczyn, A. D., Tadmor, R., Fullerton, B. C., Levine, R. A., and Furst, M. Effects of localized pontine lesions on auditory brain-stem evoked potentials and binaural processing in humans. Electroencephalogr.Clin.Neurophysiol. 108: 511-520, 1998.

125. Rall, W. Distinguishing theoretical synaptic potentials computed for different soma-dendritic distributions of synaptic input. J.Neurophysiol. 30: 1138-1168, 1967.

126. Rask-Andersen, H., Tylstedt, S., Kinnefors, A., and Illing, R. Synapses on human spiral ganglion cells: a transmission electron microscopy and immunohistochemical study. Hear.Res. 141: 1-11, 2000.

127. Rathouz, M. and Trussell, L. Characterization of outward currents in neurons of the avian nucleus magnocellularis. J.Neurophysiol. 80: 2824-2835, 1998.

128. Rayleigh, L. Acoustical observations. London, Edinburg, and Dublin Philosophical Magazine and Journal of Science 3: 456-464, 1877.

129. Rayleigh, L. On our perception of sound direction. London, Edinburg, and Dublin Philosophical Magazine and Journal of Science 13: 214-232, 1907.

130. Rizzuto, R., Pinton, P., Carrington, W., Fay, F. S., Fogarty, K. E., Lifshitz, L. M., Tuft, R. A., and Pozzan, T. Close contacts with the endoplasmic reticulum as determinants of mitochondrial Ca2+ responses. Science 280: 1763-1766, 1998.

131. Roberts, R. C. and Ribak, C. E. GABAergic neurons and axon terminals in the brainstem auditory nuclei of the gerbil. J.Comp Neurol. 258: 267-280, 1987. 
132. Roth, G. L., Aitkin, L. M., Andersen, R. A., and Merzenich, M. M. Some features of the spatial organization of the central nucleus of the inferior colliculus of the cat. J.Comp Neurol. 182: 661-680, 1978.

133. Rothman, J. S., Young, E. D., and Manis, P. B. Convergence of auditory nerve fibers onto bushy cells in the ventral cochlear nucleus: implications of a computational model. J.Neurophysiol. 70: 2562-2583, 1993.

134. Rowland, K. C., Irby, N. K., and Spirou, G. A. Specialized synapse-associated structures within the calyx of Held. J.Neurosci. 20: 9135-9144, 2000.

135. Russell, F. and Moore, D. Ultrastructural transynaptic effects of unilateral cochlear ablation in the gerbil medial superior olive. Hear.Res. 173: 43, 2002.

136. Ryugo, D. K., Pongstaporn, T., Huchton, D. M., and Niparko, J. K. Ultrastructural analysis of primary endings in deaf white cats: morphologic alterations in endbulbs of Held. J Comp Neurol. 385: 230-244, 1997.

137. Ryugo, D. K. and Sento, S. Synaptic connections of the auditory nerve in cats: relationship between endbulbs of held and spherical bushy cells. J Comp Neurol. 305: 35-48, 1991.

138. Ryugo, D. K., Wu, M. M., and Pongstaporn, T. Activity-related features of synapse morphology: a study of endbulbs of held. J.Comp Neurol. 365: 141-158, 1996.

139. Saldana, E. and Berrebi, A. S. Anisotropic organization of the rat superior paraolivary nucleus. Anat Embryol.(Berl) 202: 265-279, 2000.

140. Sanes, D. H. The development of synaptic function and integration in the central auditory system. J.Neurosci. 13: 2627-2637, 1993. 
141. Schikorski, T. and Stevens, C. F. Quantitative ultrastructural analysis of hippocampal excitatory synapses. J.Neurosci. 17: 5858-5867, 1997.

142. Schneggenburger, R., Meyer, A. C., and Neher, E. Released fraction and total size of a pool of immediately available transmitter quanta at a calyx synapse. Neuron 23: 399-409, 1999.

143. Schwartz, I. R. Axonal endings in the cat medial superior olive: coated vesicles and intercellular substance. Brain Res. 46: 187-202, 1972.

144. Schwartz, I. R. Axonal organization in the cat medial superior olivary nucleus. In Neff, W. D. ed. Contributions to Sensory Physiology. New York, Academic. 1984, 99-129.

145. Sento, S. and Ryugo, D. K. Endbulbs of held and spherical bushy cells in cats: morphological correlates with physiological properties. J.Comp Neurol. 280: 553$562,1989$.

146. Serafini, T. Finding a partner in a crowd: neuronal diversity and synaptogenesis. Cell 98: 133-136, 1999.

147. Sivaramakrishnan, S. and Oliver, D. L. Distinct K currents result in physiologically distinct cell types in the inferior colliculus of the rat. J.Neurosci. 21: 2861-2877, 2001.

148. Smith, A. J., Owens, S., and Forsythe, I. D. Characterisation of inhibitory and excitatory postsynaptic currents of the rat medial superior olive. J Physiol $529 \mathrm{Pt}$ 3: 681-698, 2000.

149. Smith, P. H. Structural and functional differences distinguish principal from nonprincipal cells in the guinea pig MSO slice. J.Neurophysiol. 73: 1653-1667, 1995. 
150. Smith, P. H., Joris, P. X., Carney, L. H., and Yin, T. C. Projections of physiologically characterized globular bushy cell axons from the cochlear nucleus of the cat. J.Comp Neurol. 304: 387-407, 1991.

151. Smith, P. H., Joris, P. X., and Yin, T. C. Projections of physiologically characterized spherical bushy cell axons from the cochlear nucleus of the cat: evidence for delay lines to the medial superior olive. J.Comp Neurol. 331: 245260, 1993.

152. Smith, P. H., Joris, P. X., and Yin, T. C. Anatomy and physiology of principal cells of the medial nucleus of the trapezoid body (MNTB) of the cat. J.Neurophysiol. 79: 3127-3142, 1998.

153. Sotelo, C., Gentschev, T., and Zamora, A. J. Gap junctions in ventral cochlear nucleus of the rat. A possible new example of electrotonic junctions in the mammalian C.N.S. Neuroscience 1: 5-7, 1976.

154. Spangler, K. M., Warr, W. B., and Henkel, C. K. The projections of principal cells of the medial nucleus of the trapezoid body in the cat. J.Comp Neurol. 238: 249262, 1985.

155. Spirou, G. A. and Berrebi, A. S. Organization of ventrolateral periolivary cells of the cat superior olive as revealed by PEP-19 immunocytochemistry and NissI stain. J.Comp Neurol. 368: 100-120, 1996.

156. Spirou, G. A. and Berrebi, A. S. Glycine immunoreactivity in the lateral nucleus of the trapezoid body of the cat. J.Comp Neurol. 383: 473-488, 1997.

157. Spirou, G. A., Brownell, W. E., and Zidanic, M. Recordings from cat trapezoid body and HRP labeling of globular bushy cell axons. J.Neurophysiol. 63: 11691190, 1990. 
158. Spirou, G. A., Rowland, K. C., and Berrebi, A. S. Ultrastructure of neurons and large synaptic terminals in the lateral nucleus of the trapezoid body of the cat. J.Comp Neurol. 398: 257-272, 1998.

159. Spitzer, M. W. and Semple, M. N. Neurons sensitive to interaural phase disparity in gerbil superior olive: diverse monaural and temporal response properties. J.Neurophysiol. 73: 1668-1690, 1995.

160. Sternberger, L. Immunocytochemistry. New York, Wiley. 1979.

161. Stevens, C. F. and Wesseling, J. F. Activity-dependent modulation of the rate at which synaptic vesicles become available to undergo exocytosis. Neuron 21: 415-424, 1998.

162. Stotler, W. An experimental study of the cells and connections of the superior olivary complex of the cat. J.Comp Neurol. 98: 401-432, 1953.

163. Swanson, R., Marshall, J., Smith, J. S., Williams, J. B., Boyle, M. B., Folander, K., Luneau, C. J., Antanavage, J., Oliva, C., Buhrow, S. A., and . Cloning and expression of cDNA and genomic clones encoding three delayed rectifier potassium channels in rat brain. Neuron 4: 929-939, 1990.

164. Takei, K., Mundigl, O., Daniell, L., and De Camilli, P. The synaptic vesicle cycle: a single vesicle budding step involving clathrin and dynamin. J.Cell Biol. 133: 1237-1250, 1996.

165. Thompson, G. C. and Masterton, R. B. Brain stem auditory pathways involved in reflexive head orientation to sound. J Neurophysiol. 41: 1183-1202, 1978.

166. Thompson, S. Phenomena of binaural audition. Philos.Mag. 6: 383-391, 1878. 
167. Tolbert, L. P. and Morest, D. K. The neuronal architecture of the anteroventral cochlear nucleus of the cat in the region of the cochlear nerve root: electron microscopy. Neuroscience 7: 3053-3067, 1982a.

168. Tolbert, L. P. and Morest, D. K. The neuronal architecture of the anteroventral cochlear nucleus of the cat in the region of the cochlear nerve root: Golgi and Nissl methods. Neuroscience 7: 3013-3030, 1982b.

169. Uchizono, K. Characteristics of excitatory and inhibitory synapses in the central nervous system of the cat. Nature 207: 642-643, 1965.

170. von Gersdorff, H. and Matthews, G. Depletion and replenishment of vesicle pools at a ribbon-type synaptic terminal. J.Neurosci. 17: 1919-1927, 1997.

171. von Gersdorff, H. and Matthews, G. Electrophysiology of synaptic vesicle cycling. Annu.Rev.Physiol 61: 725-752, 1999.

172. von Gersdorff, H., Vardi, E., Matthews, G., and Sterling, P. Evidence that vesicles on the synaptic ribbon of retinal bipolar neurons can be rapidly released. Neuron 16: 1221-1227, 1996.

173. Wang, L. Y., Gan, L., Forsythe, I. D., and Kaczmarek, L. K. Contribution of the Kv3.1 potassium channel to high-frequency firing in mouse auditory neurones. $J$ Physiol 509 ( Pt 1): 183-194, 1998.

174. Wang, L. Y. and Kaczmarek, L. K. High-frequency firing helps replenish the readily releasable pool of synaptic vesicles. Nature 394: 384-388, 1998.

175. Warr, W. B. Fiber degeneration following lesions in the multipolar and globular cell areas in the ventral cochlear nucleus of the cat. Brain Res. 40: 247-270, 1972. 
176. Wenthold, R. J., Huie, D., Altschuler, R. A., and Reeks, K. A. Glycine immunoreactivity localized in the cochlear nucleus and superior olivary complex. Neuroscience 22: 897-912, 1987.

177. Werkman, T. R., Kawamura, T., Yokoyama, S., Higashida, H., and Rogawski, M. A. Charybdotoxin, dendrotoxin and mast cell degranulating peptide block the voltage-activated $\mathrm{K}+$ current of fibroblast cells stably transfected with NGK1 (Kv1.2) K+ channel complementary DNA. Neuroscience 50: 935-946, 1992.

178. Wu, L. G. and Borst, J. G. The reduced release probability of releasable vesicles during recovery from short-term synaptic depression. Neuron 23: 821-832, 1999.

179. Wu, S. H. and Kelly, J. B. Response of neurons in the lateral superior olive and medial nucleus of the trapezoid body to repetitive stimulation: intracellular and extracellular recordings from mouse brain slice. Hear.Res. 68: 189-201, 1993.

180. Wu, S. H. and Oertel, D. Intracellular injection with horseradish peroxidase of physiologically characterized stellate and bushy cells in slices of mouse anteroventral cochlear nucleus. J Neurosci. 4: 1577-1588, 1984.

181. Yin, T. C. and Chan, J. C. Interaural time sensitivity in medial superior olive of cat. J.Neurophysiol. 64: 465-488, 1990.

182. Yost, W. A. Discriminations of interaural phase differences. J Acoust.Soc.Am. 55: 1299-1303, 1974.

183. Zenisek, D. and Matthews, G. The role of mitochondria in presynaptic calcium handling at a ribbon synapse. Neuron 25: 229-237, 2000.

184. Zhang, S. and Trussell, L. O. A characterization of excitatory postsynaptic potentials in the avian nucleus magnocellularis. J Neurophysiol. 72: 705-718, 1994. 
185. Zwislocki, J. and Feldman, R. Just noticeable differences in dichotic phase. J Acoust.Soc.Am. 28: 860-864, 1956. 